\title{
Highly Efficient and Selective Synthesis of Conjugated Triynes and Higher Oligoynes of Biological and Materials Chemical Interest via Palladium-Catalyzed Alkynyl-Alkenyl Coupling
}

Estelle Métay, Qian Hu, and Ei-ichi Negishi.

\section{Supporting Information}

General. All experiments were conducted under argon atmosphere. THF was dried and distilled from sodium and benzophenone. $\mathrm{ZnBr}_{2}$ was flame-dried under vacuum prior to use. Flash chromatographic purification was carried out on 230-400 mesh silica gel 60. Gas chromatography was performed on an HP 6890 Gas Chromatograph using a HP-5 capillary column $(30 \mathrm{~m} \times 0.32 \mathrm{~mm}, 0.5 \mu \mathrm{m}$ film $)$ with appropriate hydrocarbons as internal standards. ${ }^{1} \mathrm{H}$ and ${ }^{13} \mathrm{C}$ NMR spectra were recorded in $\mathrm{CDCl}_{3}$ or acetone- $d_{6}$ on a Varian Inova-300 or a Brucker ARX-400 spectrometer. IR spectra were recorded on a Perkin-Elmer Spectrum 2000 FT-IR spectromer. LRMS and HRMS data were obtained on Hewlett Packard 5995 GC-MS and Kratos MS-50 mass spectrometers, respectively. $(E)-\mathrm{ICH}=\mathrm{CHCl}^{1}{ }^{1}(E)-\mathrm{ICH}=\mathrm{CHCH}_{2} \mathrm{OTBS},{ }^{2}$ (E) $-\mathrm{ICH}=\mathrm{CHCH}_{2} \mathrm{OH},{ }^{3}(E)-\mathrm{BrCH}=\mathrm{CHCO}_{2} \mathrm{Et}^{4}$ were prepared according to the literature. Prepared compounds were stored in the freezer at $-20^{\circ} \mathrm{C}$ for 1 month without observing any degradation.

Representative Procedure A: (E)-1-Chloro-4-phenyl-1-buten-3-yne (3a). ${ }^{5}$ To a solution of phenylacetylene (1.02 g, $10 \mathrm{mmol})$ in THF $(15 \mathrm{~mL})$ was added ${ }^{n} \mathrm{BuLi}(2.5 \mathrm{M}$ in hexane, $0.8 \mathrm{~mL}, 10 \mathrm{mmol})$ at $-78{ }^{\circ} \mathrm{C}$. The reaction mixture was stirred at $-78{ }^{\circ} \mathrm{C}$ for $30 \mathrm{~min}$ and treated with a solution of anhydrous $\mathrm{ZnBr}_{2}(1.35 \mathrm{~g}, 6.0 \mathrm{mmol})$ in THF $(10 \mathrm{~mL})$. (E)-1-Iodo-2-chloroethylene $(1.88 \mathrm{~g}, 10 \mathrm{mmol})$ and $\mathrm{Pd}\left(\mathrm{PPh}_{3}\right)_{4}$ (231 mg, $200 \mu \mathrm{mol})$ in THF $(5 \mathrm{~mL})$ were added to the reaction mixture at $0{ }^{\circ} \mathrm{C}$, which was then stirred at $23{ }^{\circ} \mathrm{C}$. After $1 \mathrm{~h} \mathrm{GLC}$ analysis indicated that the starting material had been completely consumed. The reaction mixture was quenched with aqueous $\mathrm{NH}_{4} \mathrm{Cl}$, extracted with ether, washed successively with saturated $\mathrm{NaHCO}_{3}$ and brine, dried over $\mathrm{MgSO}_{4}$, filtered, and concentrated. Purification by column chromatography (silica gel, pentane) afforded $1.49 \mathrm{~g}(92 \%)$ of the title compound: colorless oil; ${ }^{1} \mathrm{H}$ NMR $\left(300 \mathrm{MHz}, \mathrm{CDCl}_{3}\right) \delta 6.33(\mathrm{~d}, J=13.4 \mathrm{~Hz}, 1 \mathrm{H}), 6.77(\mathrm{~d}, J=13.4 \mathrm{~Hz}, 1 \mathrm{H}), 7.45-7.5(\mathrm{~m}, 3 \mathrm{H}), 7.6-7.65(\mathrm{~m}$,

\footnotetext{
${ }^{1}$ Negishi, E.; Okukado, N.; Lovich, S.; Luo, F. T. J. Org. Chem. 1984, 49, 2629.

2 (a) Xu, C.; Negishi, E. Tetrahedron Lett. 1999, 40, 431. (b) Huang, Z.; Negishi, E. Org. Lett. 2006, 8, 3675.

${ }^{3}$ Yin, N.; Wang, G.; Qian, M.; Negishi, E. Angew. Chem. Int. Ed. 2006, 45, 2916.

${ }^{4}$ Wier, J. R.; Patel, B. A.; Heck, R. F. J. Org. Chem. 1980, 45, 4926. Pattenden, G.; Walker B. J. J. Chem. Soc. 1969, 531.

${ }^{5}$ Negishi, E.; Hata, M.; Xu, C. Org. Lett. 2000, 2, 3687-3689.
} 
2H) ppm; ${ }^{13} \mathrm{C} \mathrm{NMR}\left(\mathrm{CDCl}_{3}, 75 \mathrm{MHz}\right) \delta 84.38,91.94,113.78,122.54,128.26(2 \mathrm{C}), 128.49,130.06,131.41$ (2C) ppm; IR (neat) 3069, 3023, 2253, 2201, 1581, $1488 \mathrm{~cm}^{-1}$; HRMS m/z calcd for $\mathrm{C}_{10} \mathrm{H}_{7} \mathrm{Cl}: 162.0236$; found: 162.0234 .

(E)-1-Chloro-4-trimethylsilyl-1-buten-3-yne (3b). ${ }^{6}$ The title compound was prepared according to Representative Procedure A: Yield 81\%; colorless oil; ${ }^{1} \mathrm{H}$ NMR $\left(\mathrm{CDCl}_{3}, 300 \mathrm{MHz}\right) \delta 0.16(\mathrm{~s}, 9 \mathrm{H}), 5.92(\mathrm{~d}, J$ $=13.5 \mathrm{~Hz}, 1 \mathrm{H}), 6.55(\mathrm{~d}, J=13.5 \mathrm{~Hz}, 1 \mathrm{H}) \mathrm{ppm} ;{ }^{13} \mathrm{C} \mathrm{NMR}\left(\mathrm{CDCl}_{3}, 75 \mathrm{MHz}\right) \delta-0.30(3 \mathrm{C}), 97.64,99.60$, $113.89,131.58 \mathrm{ppm}$.

(E)-1-Chloro-1-penten-3-yne (3c). The title compound was prepared according to Representative Procedure A: Yield 67\%; colorless oil; ${ }^{1} \mathrm{H}$ NMR $\left(\mathrm{CDCl}_{3}, 300 \mathrm{MHz}\right) \delta 1.91(\mathrm{~d}, J=2.3 \mathrm{~Hz}, 3 \mathrm{H}), 5.87$ (dq, $J$ $=13.5$ and $2.3 \mathrm{~Hz}, 1 \mathrm{H}), 6.41(\mathrm{~d}, J=13.5 \mathrm{~Hz}, 1 \mathrm{H}) \mathrm{ppm} ;{ }^{13} \mathrm{C} \mathrm{NMR}\left(\mathrm{CDCl}_{3}, 75 \mathrm{MHz}\right) \delta$ 4.01, 74.84, 88.71, 114.17, 128.74 ppm; IR (neat) 3080, 3023, 2914, 2847, $2222 \mathrm{~cm}^{-1}$; HRMS m/z calcd for $\mathrm{C}_{5} \mathrm{H}_{5} \mathrm{Cl}$ : 100.0080 ; found: 100.0080 .

Representative Procedure A' (E)-1-Chloro-6-phenyl-1-hexene-3,5-diyne (4a). To a solution of (E)-1-Chloro-4-phenyl-1-butene-3-yne (1.49 g, $9.2 \mathrm{mmol})$ in THF $(27 \mathrm{~mL})$ was added ${ }^{n} \mathrm{BuLi}(2.5 \mathrm{M}$ in hexane, $7.4 \mathrm{~mL}, 18.4 \mathrm{mmol}$ ) at $-78{ }^{\circ} \mathrm{C}$. The reaction mixture was first stirred $-78^{\circ} \mathrm{C}$ for $30 \mathrm{~min}$ and then at $-30{ }^{\circ} \mathrm{C}$ for $30 \mathrm{~min}$ and treated with anhydrous $\mathrm{ZnBr}_{2}(1.24 \mathrm{~g}, 5.5 \mathrm{mmol})$ in THF $(10 \mathrm{~mL})$. (E)-Iodochloroethylene $(1.74 \mathrm{~g}, 9.2 \mathrm{mmol})$ and $\mathrm{Pd}\left(\mathrm{PPh}_{3}\right)_{4}(213 \mathrm{mg}, 0.18 \mathrm{mmol})$ in THF $(10 \mathrm{~mL})$ were added to the reaction at $0{ }^{\circ} \mathrm{C}$ which was then stirred at $0{ }^{\circ} \mathrm{C}$. After $6 \mathrm{~h}$ GLC analysis indicated that the starting material had been completely consumed. The reaction mixture was quenched with aqueous $\mathrm{NH}_{4} \mathrm{Cl}$, extracted with ether, washed successively with saturated $\mathrm{NaHCO}_{3}$ and brine, dried over $\mathrm{MgSO}_{4}$, filtered, and concentrated. Purification by column chromatography (silica gel, pentane) afforded $1.33 \mathrm{~g}(78 \%)$ of the title compound: $\mathrm{mp} 41-42{ }^{\circ} \mathrm{C}$; colorless solid; ${ }^{1} \mathrm{H} \mathrm{NMR}\left(\mathrm{CDCl}_{3}, 300 \mathrm{MHz}\right) \delta 6.15(\mathrm{~d}, J=13.5 \mathrm{~Hz}, 1 \mathrm{H})$, $6.80(\mathrm{~d}, J=13.5 \mathrm{~Hz}, 1 \mathrm{H}), 7.4-7.5(\mathrm{~m}, 3 \mathrm{H}), 7.6-7.65(\mathrm{~m}, 2 \mathrm{H}) \mathrm{ppm} ;{ }^{13} \mathrm{C} \mathrm{NMR}\left(\mathrm{CDCl}_{3}, 75 \mathrm{MHz}\right) \delta 73.49$, 76.52 (2C), 82.56, 112.91, 121.36, 128.41 (2C), 129.39, 132.48 (2C), 133.77 ppm; IR (neat) 3069, 3049, 2304, 2217, 1576, 1488, $1439 \mathrm{~cm}^{-1}$; HRMS m/z calcd for $\mathrm{C}_{12} \mathrm{H}_{7} \mathrm{Cl}$ : 186.0236; found: 186.0235 .

(1E,5E)-1-Chloro-1,5-heptadien-3-yne (3d). The title compound was prepared according to Representative Procedure $\mathrm{A}^{\prime}$ from $(E)$-1,1-dibromo-1,3-pentadiene except that the reaction mixture was

\footnotetext{
${ }^{6}$ Alami, M.; Gueugnot, S.; Domingues, E.; Linstrumelle, G.; Tetrahedron 1995, 51, 1209.
} 
stirred at $-78^{\circ} \mathrm{C}$ for $1 \mathrm{~h}$ after addition of ${ }^{n} \mathrm{BuLi}$ : Yield $74 \%$; colorless oil; ${ }^{1} \mathrm{H}$ NMR $\left(\mathrm{CDCl}_{3}, 300 \mathrm{MHz}\right) \delta 1.75$ (dd, $J=6.9$ and $1.8 \mathrm{~Hz}, 3 \mathrm{H}), 5.54(\mathrm{~d}, J=15.8 \mathrm{~Hz}, 1 \mathrm{H}), 5.98(\mathrm{~d}, J=13.5 \mathrm{~Hz}, 1 \mathrm{H}), 6.12(\mathrm{dq}, J=15.8$ and 6.9 $\mathrm{Hz}, 1 \mathrm{H}), 6.43(\mathrm{~d}, J=13.5 \mathrm{~Hz}, 1 \mathrm{H}) \mathrm{ppm} ;{ }^{13} \mathrm{C} \mathrm{NMR}\left(\mathrm{CDCl}_{3}, 75 \mathrm{MHz}\right) \delta 18.51,82.73,90.84,110.34,113.93$, 128.97, 140.29 ppm; IR (neat) 3073, 3028, 2960, 2932, $2193 \mathrm{~cm}^{-1}$; HRMS m/z calcd for $\mathrm{C}_{7} \mathrm{H}_{7} \mathrm{Cl}$ : 126.0236 ; found: 126.0235 .

(1E,5E)-1-(2-Furyl)-6-chloro-1,5-hexadien-3-yne (3e). The title compound was prepared according to Representative Procedure A' from 1,1-dibromo-4-(2-furyl)-1,3-butadiene except that the reaction mixture was stirred at $-78{ }^{\circ} \mathrm{C}$ for $1 \mathrm{~h}$ after addition of ${ }^{n} \mathrm{BuLi}$ : Yield $70 \%$; colorless oil; ${ }^{1} \mathrm{H}$ NMR $\left(\mathrm{CDCl}_{3}, 300 \mathrm{MHz}\right) \delta$ 6.0-6.2 (m, 2H), 6.3-6.35 (m, 2H), $6.49(\mathrm{~d}, J=13.5 \mathrm{~Hz}, 1 \mathrm{H}), 6.66(\mathrm{~d}, J=15.8 \mathrm{~Hz}, 1 \mathrm{H}), 7.32(\mathrm{~s}, 1 \mathrm{H}) \mathrm{ppm} ;{ }^{13} \mathrm{C}$ $\mathrm{NMR}\left(\mathrm{CDCl}_{3}, 75 \mathrm{MHz}\right) \delta 87.64,91.65,105.41,110.49,111.81,113.95,128.63,129.61,143.11,151.87$ ppm; HRMS m/z calcd for $\mathrm{C}_{10} \mathrm{H}_{7} \mathrm{ClO}$ : 178.0185; found: 178.0187 .

(E)-1-Chloro-6-trimethylsilyl-1-hexene-3,5-diyne (4b). The title compound was prepared according to Representative Procedure A': Yield 80\%; colorless oil; ${ }^{1} \mathrm{H}$ NMR $\left(\mathrm{CDCl}_{3}, 300 \mathrm{MHz}\right) \delta 0.19(\mathrm{~s}, 9 \mathrm{H}), 5.95$ $(\mathrm{d}, J=13.5 \mathrm{~Hz}, 1 \mathrm{H}), 6.67(\mathrm{~d}, J=13.5 \mathrm{~Hz}, 1 \mathrm{H}) \mathrm{ppm} ;{ }^{13} \mathrm{C} \mathrm{NMR}\left(\mathrm{CDCl}_{3}, 75 \mathrm{MHz}\right) \delta-0.54(3 \mathrm{C}), 71.64,76.72$, 87.30, 91.94, 112.60, 134.41 ppm; IR (neat) 3073, 3024, 2961, 2900, 2202, $2102 \mathrm{~cm}^{-1}$; HRMS m/z calcd for $\mathrm{C}_{9} \mathrm{H}_{11} \mathrm{ClSi}$ : 182.0319 ; found: 180.0320 .

(E)-1-Chloro-1-heptene-3,5-diyne (4c). The title compound was prepared according to Representative Procedure A': Yield 73\%; mp 39-40 ${ }^{\circ} \mathrm{C}$; colorless solid; ${ }^{1} \mathrm{H}$ NMR $\left(\mathrm{CDCl}_{3}, 300 \mathrm{MHz}\right) \delta 2.03$ (s, 3H), $6.98(\mathrm{~d}, J=13.5 \mathrm{~Hz}, 1 \mathrm{H}), 6.67(\mathrm{~d}, J=13.5 \mathrm{~Hz}, 1 \mathrm{H}) \mathrm{ppm} ;{ }^{13} \mathrm{C} \mathrm{NMR}\left(\mathrm{CDCl}_{3}, 75 \mathrm{MHz}\right): \delta 4.48$, 63.98, 69.11, 77.00, 81.49, 112.94, 133.13 ppm; IR (neat) 3074, 3028, 2910, 2839, $2232 \mathrm{~cm}^{-1}$; HRMS m/z calcd for $\mathrm{C}_{7} \mathrm{H}_{5} \mathrm{Cl}$ : 124.0080; found: 124.0077 .

(E)-2-Decene-4,6,8-triyn-1-ol (5e). ${ }^{7}$ The title compound was prepared according to Representative Procedure $\mathrm{A}^{\prime}$ except after except that the reaction mixture was stirred at $-78^{\circ} \mathrm{C}$ for $1 \mathrm{~h}$ after addition of ${ }^{n} \mathrm{BuLi}$, and that $(E)-\mathrm{ICH}=\mathrm{CHCH}_{2} \mathrm{OZnBr}$ was used instead of $(E)$-1-iodo-2-chloroethylene: Yield $69 \%$; mp 127-128 ${ }^{\circ} \mathrm{C}$; brown solid; ${ }^{1} \mathrm{H}$ NMR (acetone- $\left.d_{6}, 300 \mathrm{MHz}\right) \delta 2.1(\mathrm{~s}, 3 \mathrm{H}), 4.25-4.3(\mathrm{~m}, 3 \mathrm{H}), 5.90(\mathrm{~d}, J=$ $14.6 \mathrm{~Hz}, 1 \mathrm{H}), 6.55-6.6(\mathrm{~m}, 1 \mathrm{H}) \mathrm{ppm} ;{ }^{13} \mathrm{C}$ NMR (acetone- $\left.d_{6}, 75 \mathrm{MHz}\right) \delta 3.42,58.73,61.48,64.20,66.62$, 73.83, 74.56, 78.94, 105.98, 149.97 ppm; IR $\left(\mathrm{CCl}_{4}\right)$ 3680-3650, 3006, 2962, 2917, 2849, 2225, $2201 \mathrm{~cm}^{-1}$; HRMS m/z calcd for $\mathrm{C}_{10} \mathrm{H}_{8} \mathrm{O}: 144.0575$; found: 144.0576 .

\footnotetext{
${ }^{7}$ Mahtabuddin, A.; Keeping, J. W.; Macrides, T. A.; Thaller, V. J. Chem. Soc. Perkin Trans 1 1978, 12, 1487.
} 
(E)-1-(tert-Butyldimethylsilyloxy)-2-decene-4,6,8-triyne (5f). The title compound was prepared according to Representative Procedure $\mathrm{A}^{\prime}$ except that the reaction mixture was stirred at $-78{ }^{\circ} \mathrm{C}$ for $1 \mathrm{~h}$ after addition of ${ }^{n} \mathrm{BuLi}$, and that $(E)$ - $\mathrm{ICH}=\mathrm{CHCH}_{2} \mathrm{OTBS}$ was used instead of $(E)$-1-iodo-2-chloroethylene: Yield 71\%; mp 50-51 ${ }^{\circ} \mathrm{C}$; yellow solid; ${ }^{1} \mathrm{H}$ NMR $\left(\mathrm{CDCl}_{3}, 300 \mathrm{MHz}\right) \delta 0.12(\mathrm{~s}, 6 \mathrm{H}), 0.96(\mathrm{~s}, 9 \mathrm{H}), 2.03(\mathrm{~s}, 3 \mathrm{H}), 4.31$ (dd, $J=3.5$ and $1.7 \mathrm{~Hz}, 2 \mathrm{H}), 5.85(\mathrm{dt}, J=15.8$ and $1.7 \mathrm{~Hz}, 1 \mathrm{H}), 6.75(\mathrm{dt}, J=15.7$ and $3.5 \mathrm{~Hz}, 1 \mathrm{H}) \mathrm{ppm} ;{ }^{13} \mathrm{C}$ NMR $\left(\mathrm{CDCl}_{3}, 75 \mathrm{MHz}\right) \delta-5.56(2 \mathrm{C}), 4.51,18.21,25.71$ (3C), 59.03, 62.65, 64.90, 66.84, 74.02, 74.70, 77.87, 106.65, 147.66 ppm; IR ( $\left(\mathrm{CCl}_{4}\right)$ 2956, 2945, 2885, 2857, 2224, 2201, $2183 \mathrm{~cm}^{-1}$; HRMS m/z calcd for $\mathrm{C}_{16} \mathrm{H}_{22} \mathrm{OSi}: 258.1440$; found: 258.1439 .

Ethyl (E)-2-Decene-4,6,8-triynoate (5g). The title compound was prepared according to Representative Procedure $\mathrm{A}^{\prime}$ except that the reaction mixture was stirred at $-78{ }^{\circ} \mathrm{C}$ for $1 \mathrm{~h}$ after addition of ${ }^{n} \mathrm{BuLi}$, and that $(E)-\mathrm{BrCH}=\mathrm{CHCO}_{2} \mathrm{Et}$ was used instead of $(E)$-1-iodo-2-chloroethylene: Yield $68 \%$; mp 64-65 ${ }^{\circ} \mathrm{C}$; yellow solid; ${ }^{1} \mathrm{H}$ NMR $\left(\mathrm{CDCl}_{3}, 400 \mathrm{MHz}\right) \delta 1.23(\mathrm{t}, J=7.1 \mathrm{~Hz}, 3 \mathrm{H}), 1.95$ (s, 3H), 4.15 (q, $J=$ $7.1 \mathrm{~Hz}, 2 \mathrm{H}), 6.29(\mathrm{~d}, J=15.9 \mathrm{~Hz}, 1 \mathrm{H}), 6.67(\mathrm{~d}, J=15.9 \mathrm{~Hz}, 1 \mathrm{H}) \mathrm{ppm} ;{ }^{13} \mathrm{C} \mathrm{NMR}\left(\mathrm{CDCl}_{3}, 100 \mathrm{MHz}\right) \delta 4.69$, $14.07,58.06,60.99,64.6,70.88,71.55,80.46,82.75,123.22,134.03,165.17 \mathrm{ppm}$; IR $\left(\mathrm{CCl}_{4}\right) 2983,2939$, 2915, 2873, 2225, 2178, $1717 \mathrm{~cm}^{-1}$; HRMS m/z calcd for $\mathrm{C}_{12} \mathrm{H}_{10} \mathrm{O}_{2}$ : 186.0681 ; found: 186.0682 .

(E)-1-Chloro-8-phenyl-1-octene-3,5,7-triyne (6a). The title compound was prepared according to Representative Procedure $\mathrm{A}^{\prime}$ except that the reaction mixture was stirred at $-78{ }^{\circ} \mathrm{C}$ for $1 \mathrm{~h}$ after addition of ${ }^{n}$ BuLi: Yield 74\%; mp 61-62 ${ }^{\circ} \mathrm{C}$; yellow solid; ${ }^{1} \mathrm{H}$ NMR $\left(\mathrm{CDCl}_{3}, 300 \mathrm{MHz}\right) \delta 6.00(\mathrm{~d}, J=13.5 \mathrm{~Hz}, 1 \mathrm{H})$, $6.74(\mathrm{~d}, J=13.5 \mathrm{~Hz}, 1 \mathrm{H}), 7.3-7.4(\mathrm{~m}, 3 \mathrm{H}), 7.5-7.55(\mathrm{~m}, 2 \mathrm{H}) \mathrm{ppm} ;{ }^{13} \mathrm{C} \mathrm{NMR}\left(\mathrm{CDCl}_{3}, 75 \mathrm{MHz}\right) \delta 65.94$, $67.54,73.55,74.22,77.00,79.25,112.32,120.57,128.40$ (2C), 129.72, 132.89 (2C), 135.56 ppm; IR (neat) 3075, 2196, 2175, 1573, $1490 \mathrm{~cm}^{-1}$; HRMS m/z calcd for $\mathrm{C}_{14} \mathrm{H}_{7} \mathrm{Cl}: 210.0236$; found: 210.0236.

(E)-1-Chloro-8-trimethylsilyl-1-octene-3,5,7-triyne (6b). The title compound was prepared according to Representative Procedure $\mathrm{A}^{\prime}$ except that the reaction mixture was stirred at $-78{ }^{\circ} \mathrm{C}$ for $1 \mathrm{~h}$ after addition of ${ }^{n} \mathrm{BuLi}$ : Yield 76\%; yellow oil; ${ }^{1} \mathrm{H}$ NMR $\left(\mathrm{CDCl}_{3}, 300 \mathrm{MHz}\right) \delta 0.19(\mathrm{~s}, 9 \mathrm{H}), 5.95(\mathrm{~d}, J=13.5 \mathrm{~Hz}$, 1H), $6.73(\mathrm{~d}, J=13.5 \mathrm{~Hz}, 1 \mathrm{H}) \mathrm{ppm} ;{ }^{13} \mathrm{C} \mathrm{NMR}\left(\mathrm{CDCl}_{3}, 75 \mathrm{MHz}\right) ; \delta-0.62(3 \mathrm{C}), 60.94,67.79,71.75,76.83$, 87.72, 89.83, 112.26, 135.95 ppm; IR (neat) $3072,3023,2962,2899,2167,2079 \mathrm{~cm}^{-1} ; \mathrm{HRMS} \mathrm{m} / \mathrm{z}$ calcd for $\mathrm{C}_{11} \mathrm{H}_{11} \mathrm{ClSi}$ : 206.0319; found: 206.0321.

(E)-1-Chloro-1-nonene-3,5,7-triyne (6c). The title compound was prepared according to Representative Procedure $\mathrm{A}^{\prime}$ except that the reaction mixture was stirred at $-78{ }^{\circ} \mathrm{C}$ for $1 \mathrm{~h}$ after addition of 
${ }^{n} \mathrm{BuLi}$ : Yield 63\%; mp 95-96 ${ }^{\circ} \mathrm{C}$; yellow solid; ${ }^{1} \mathrm{H}$ NMR $\left(\mathrm{CDCl}_{3}, 300 \mathrm{MHz}\right) \delta 1.97$ (s, 3H), $6.93(\mathrm{~d}, J=$ $13.5 \mathrm{~Hz}, 1 \mathrm{H}), 6.70(\mathrm{~d}, J=13.5 \mathrm{~Hz}, 1 \mathrm{H}) \mathrm{ppm} ;{ }^{13} \mathrm{C} \mathrm{NMR}\left(\mathrm{CDCl}_{3}, 75 \mathrm{MHz}\right) \delta 4.58,58.33,64.59,68.30$, 70.09, 76.89, 78.99, 112.29, 135.15 ppm; IR $\left(\mathrm{CCl}_{4}\right): 3074,3024,2915,2842,2226 \mathrm{~cm}^{-1}$; HRMS m/z calcd for $\mathrm{C}_{9} \mathrm{H}_{5} \mathrm{Cl}: 148.0080$; found: 148.0079 .

(2E, 10E)-1-(tert-Butyldimethylsilyloxy)-2,10-diene-4,6,8-dodecatriyne (8). The title compound was prepared according to Representative Procedure $\mathrm{A}^{\prime}$ except that the reaction mixture was stirred at $-78^{\circ} \mathrm{C}$ for $1 \mathrm{~h}$ after addition of ${ }^{n} \mathrm{BuLi}$, and that $(E)-\mathrm{ICH}=\mathrm{CHCH}_{2} \mathrm{OTBS}$ was used instead of (E)-1-iodo-2-chloroethylene: Yield 71\%; colorless oil; ${ }^{1} \mathrm{H}$ NMR $\left(\mathrm{CDCl}_{3}, 300 \mathrm{MHz}\right) \delta 0.04$ (s, 6H), 0.88 (s, 9H), $1.81(\mathrm{dd}, J=6.3$ and $1.8 \mathrm{~Hz}, 3 \mathrm{H}), 4.24(\mathrm{dd}, J=6.3$ and $1.8 \mathrm{~Hz}, 2 \mathrm{H}), 5.52(\mathrm{dd}, J=15.9$ and $1.8 \mathrm{~Hz}, 1 \mathrm{H})$, $5.81(\mathrm{td}, J=15.3$ and $2.1 \mathrm{~Hz}, 1 \mathrm{H}), 6.3-6.45(\mathrm{~m}, 2 \mathrm{H}) \mathrm{ppm} ;{ }^{13} \mathrm{C} \mathrm{NMR}\left(\mathrm{CDCl}_{3}, 75 \mathrm{MHz}\right) \delta-5.51(2 \mathrm{C}), 18.21$, 18.97, 25.74 (3C), 62.68, 65.49, 66.16, 72.87, 74.67, 76.92, 77.56, 106.73, 109.31, 145.53, 147.74 ppm; HRMS m/z calcd for $\mathrm{C}_{18} \mathrm{H}_{24} \mathrm{OSi}$ : 284.1596; found: 284.1598 .

(1E,7E)-1-Chloro-1,7-nonadiene-3,5-diyne (9). The title compound was prepared according to Representative Procedure $\mathrm{A}^{\prime}$, except that the reaction mixture was stirred at $-78^{\circ} \mathrm{C}$ for $1 \mathrm{~h}$ after addition of ${ }^{n} \mathrm{BuLi}$; Yield 68\%; yellow solid; ${ }^{1} \mathrm{H}$ NMR $\left(\mathrm{CDCl}_{3}, 300 \mathrm{MHz}\right) \delta 1.81(\mathrm{dd}, J=6.9$ and $1.8 \mathrm{~Hz}, 3 \mathrm{H}), .5 .54(\mathrm{~d}, J$ $=15.9 \mathrm{~Hz}, 1 \mathrm{H}), 5.99(\mathrm{~d}, J=13.5 \mathrm{~Hz}, 1 \mathrm{H}), 6.32(\mathrm{dq}, J=15.9$ and $6.9 \mathrm{~Hz}, 1 \mathrm{H}), 6.62(\mathrm{~d}, J=13.5 \mathrm{~Hz}, 1 \mathrm{H}) \mathrm{ppm}$; ${ }^{13} \mathrm{C} \mathrm{NMR}\left(\mathrm{CDCl}_{3}, 75 \mathrm{MHz}\right) \delta 18.92,71.81,75.38,76.68,81.79,109.55,113.01,133.25,144.39$ ppm; IR (neat): 3076, 3030, 2923, 2851, $2201 \mathrm{~cm}^{-1}$; HRMS m/z calcd for $\mathrm{C}_{9} \mathrm{H}_{7} \mathrm{Cl}: 150.0236$; found: 150.0239 .

\section{Representative Procedure B, 1-Phenyl-1,3-pentadiyne. ${ }^{1}$ To a solution of $^{2}$,} (E)-1-Chloro-4-phenyl-1-butene-3-yne $(324 \mathrm{mg}, 2 \mathrm{mmol})$ in THF $(20 \mathrm{~mL})$ was added ${ }^{n}$ BuLi $(2.5 \mathrm{M}$ in hexane, $1.6 \mathrm{~mL}, 4 \mathrm{mmol}$,) at $-78^{\circ} \mathrm{C}$, the reaction was stirred at $-78^{\circ} \mathrm{C}$ for $1 \mathrm{~h}$. Iodomethane $(852 \mathrm{mg}, 6 \mathrm{mmol})$ was then added to the reaction mixture which was warmed at $23{ }^{\circ} \mathrm{C}$ and kept at this temperature overnight. The reaction mixture was quenched with aqueous $\mathrm{NH}_{4} \mathrm{Cl}$, extracted with ether, washed successively with saturated $\mathrm{NaHCO}_{3}$ and brine, dried over $\mathrm{MgSO}_{4}$, filtered, and concentrated. Purification by column chromatography (silica gel, pentane) afforded $257 \mathrm{mg}$ (92\%) of the title compound: colorless oil; ${ }^{1} \mathrm{H}$ NMR $\left(\mathrm{CDCl}_{3}, 300 \mathrm{MHz}\right) \delta 1.82(\mathrm{~s}, 3 \mathrm{H}), 7.3-7.35(\mathrm{~m}, 3 \mathrm{H}), 7.45-7.5(\mathrm{~m}, 2 \mathrm{H}) \mathrm{ppm} ;{ }^{13} \mathrm{C} \mathrm{NMR}\left(\mathrm{CDCl}_{3}, 75 \mathrm{MHz}\right) \delta$ $4.43,64.28,74.02,74.38,80.28,121.89,128.23,128.74,132.38 \mathrm{ppm}$. 
1,7-Diphenyl-2,4,6-heptatriyn-1-ol (5a). ${ }^{8}$ The title compound was prepared according to Representative Procedure B except that benzaldehyde (1.2 eq) was used instead of iodomethane: Yield 86\%; red brown oil; ${ }^{1} \mathrm{H}$ NMR $\left(\mathrm{CDCl}_{3}, 300 \mathrm{MHz}\right) \delta 3.29$, (bs, $\left.1 \mathrm{H}\right), 5.52(\mathrm{~s}, 1 \mathrm{H}), 7.3-7.6(\mathrm{~m}, 10 \mathrm{H}) \mathrm{ppm} ;{ }^{13} \mathrm{C}$ NMR $\left(\mathrm{CDCl}_{3}, 75 \mathrm{MHz}\right) \delta 64.20,64.79,65.83,71.41,74.11,77.59,79.05,120.46,126.61(2 \mathrm{C}), 128.37$ (2C), 128.68 (3C), 129.72, 132.89 (2C), 139.01 ppm; IR (neat) 3652-3090, 3064, 3033, 2976, 2874, 2191, 1600, $1491 \mathrm{~cm}^{-1}$; HRMS m/z calcd for $\mathrm{C}_{19} \mathrm{H}_{12} \mathrm{O}: 256.0888$; found: 256.0888 .

1-(tert-Butyldimethylsilyl)-6-phenyl-1,3,5-hexatriyne (5b). The title compound was prepared according to representative Procedure B except that TBSCl (1.2 eq) was used instead of iodomethane: Yield 80\%; colorless oil; ${ }^{1} \mathrm{H}$ NMR $\left(\mathrm{CDCl}_{3}, 300 \mathrm{MHz}\right) \delta 0.18(\mathrm{~s}, 6 \mathrm{H}), 0.98(\mathrm{~s}, 9 \mathrm{H}), 7.3-7.35(\mathrm{~m}, 3 \mathrm{H})$, 7.5-7.55 (m, 2H) ppm; ${ }^{13} \mathrm{C}$ NMR $\left(\mathrm{CDCl}_{3}, 75 \mathrm{MHz}\right) \delta-4.97$ (2C), 16.79, 25.95 (3C), 61.18, 67.01, 74.29, 76.74, 87.85, 88.68, 120.75, 128.45 (2C), 129.73, 133.02 (2C) ppm; IR (neat) 2954, 2929, 2884, 2858, 2175, 2075, 1595, $1490 \mathrm{~cm}^{-1}$; HRMS m/z calcd for $\mathrm{C}_{18} \mathrm{H}_{20} \mathrm{Si}$ : 264.1334; found: 264.1336 .

1-Phenyl-1,3,5-heptatriyne (5c). ${ }^{9}$ The title compound was prepared according to Representative Procedure B: Yield 94\%; mp 57-58 ${ }^{\circ} \mathrm{C}$; colorless solid; ${ }^{1} \mathrm{H}$ NMR $\left(\mathrm{CDCl}_{3}, 300 \mathrm{MHz}\right) \delta 1.99$ (s, 3H), 7.3-7.4 (m, 3H), 7.5-7.52 (m, 2H) ppm; ${ }^{13} \mathrm{C} \mathrm{NMR}\left(\mathrm{CDCl}_{3}, 75 \mathrm{MHz}\right) \delta$ 4.50, 58.89, 64.84, 67.43, 74.50, 75.15, 78.35, 120.89, 128.35 (2C), 129.42, 132.79 (2C) ppm; IR $\left(\mathrm{CCl}_{4}\right)$ 3064, 3038, 2217, 1491, $1439 \mathrm{~cm}^{-1}$; HRMS m/z calcd for $\mathrm{C}_{13} \mathrm{H}_{8}: 164.0626$; found: 164.0625 .

1-Trimethylsilyl-1,3,5-heptatriyne (5d). ${ }^{10}$ The title compound was prepared according to Representative Procedure B: Yield 81\%; yellow oil; ${ }^{1} \mathrm{H}$ NMR $\left(\mathrm{CDCl}_{3}, 300 \mathrm{MHz}\right) \delta 0.19(\mathrm{~s}, 9 \mathrm{H}), 1.96$ (s, $3 \mathrm{H}) \mathrm{ppm} ;{ }^{13} \mathrm{C} \mathrm{NMR}\left(\mathrm{CDCl}_{3}, 75 \mathrm{MHz}\right) \delta-0.54$ (3C), 4.51, 59.26, 62.51, 64.70, 76.56, 85.14, 88.31 ppm; IR (neat) 2900, 2842, 2218, 2169, $2082 \mathrm{~cm}^{-1}$; HRMS m/z calcd for $\mathrm{C}_{10} \mathrm{H}_{12} \mathrm{Si}$ : 160.0708; found: 160.0707 .

1-Phenyl-1,3,5,7-nonatetrayne (7a). The title compound was prepared according to Representative Procedure B: Yield 82\%; mp 83-84 ${ }^{\circ} \mathrm{C}$ (decomposition); yellow solid; ${ }^{1} \mathrm{H}$ NMR $\left(\mathrm{CDCl}_{3}, 300 \mathrm{MHz}\right) \delta 2.07$ (s, 3H), 7.35-7.45 (m, 3H), 7.55-7.6 (m, 2H) ppm; ${ }^{13} \mathrm{C} \mathrm{NMR}\left(\mathrm{CDCl}_{3}, 75 \mathrm{MHz}\right) \delta$ 4.70, 59.68, 60.18, 64.76, 64.96, 67.57, 74.47, 75.96, 77.87, 120.58, 128.49 (2C), 129.87, 133.18 (2C) ppm; IR ( $\left.\mathrm{CCl}_{4}\right)$ 3053, 3038, 2222, 1491, $1441 \mathrm{~cm}^{-1}$; HRMS m/z calcd for $\mathrm{C}_{15} \mathrm{H}_{8}$ : 188.0626; found: 188.0628 .

\footnotetext{
${ }^{8}$ Morisaki, Y.; Luu, T.; Tykwinski, R. R. Org. Lett. 2006, 8, 689.

${ }^{9}$ Fiandanese, V.; Bottalico, D.; Marchese, G.; Punzi, A. Tetrahedron 2006, 62, 5126.

${ }^{10}$ Mukai, C.; Miyakoshi, N.; Hanaoka, M. J. Org. Chem. 2001, 66, 5875.
} 
1-Trimethylsilyl-8-phenyl-1,3,5,7-octatetrayne (7b). The title compound was prepared according to Representative Procedure B except that chlorotrimethylsilane (1.2 eq) was used instead of iodomethane: Yield 84\%; colorless oil; ${ }^{1} \mathrm{H}$ NMR $\left(\mathrm{CDCl}_{3}, 300 \mathrm{MHz}\right) \delta 0.34(\mathrm{~s}, 9 \mathrm{H}), 7.4-7.51(\mathrm{~m}, 3 \mathrm{H})$, 7.6-7.65 (m, 2H) ppm; ${ }^{13} \mathrm{C} \mathrm{NMR}\left(\mathrm{CDCl}_{3}, 75 \mathrm{MHz}\right) \delta-0.46$ (3C) 61.84, 62.15, 64.00, 67.12, 74.31, 76.86, 87.89, 88.76, 120.20, 128.49 (2C), 130.00, 133.18 (2C) ppm; IR (neat) 2961, 2899, 2195, 2132, 2059, 1491, $1442 \mathrm{~cm}^{-1}$; HRMS m/z calcd for $\mathrm{C}_{17} \mathrm{H}_{14} \mathrm{Si}: 246.0865$; found: 246.0867 .

(E)-1,1-Dibromo-1,3-pentadiene. ${ }^{11}$ This experiment was carried out in the dark by covering the reaction flask with aluminum foil. To a solution of $(E)$-crotonaldehyde $(0.41 \mathrm{~mL}, 5.0 \mathrm{mmol})$ and tetrabromomethane $(3.32 \mathrm{~g}, 10 \mathrm{mmol})$ in $\mathrm{CH}_{2} \mathrm{Cl}_{2}(30 \mathrm{~mL})$ was added $\mathrm{PPh}_{3}(5.24 \mathrm{~g}, 20 \mathrm{mmol})$ in several portions at $0{ }^{\circ} \mathrm{C}$ over a period of $3 \mathrm{~min}$, and the bright orange mixture was stirred for $10 \mathrm{~min}$ at $0{ }^{\circ} \mathrm{C}$. The reaction mixture was diluted with pentane $(200 \mathrm{~mL})$, filtered through a layer of silica gel and concentrated. Purification by column chromatography (silica gel, pentane) afforded $960 \mathrm{mg}(85 \%)$ of the title compound: colorless oil; ${ }^{1} \mathrm{H}$ NMR $\left(\mathrm{CDCl}_{3}, 300 \mathrm{MHz}\right) \delta 1.75(\mathrm{dd}, J=1.5$ and $6.8 \mathrm{~Hz}, 3 \mathrm{H}), 5.85-5.95(\mathrm{~m}, 1 \mathrm{H}), 6.0-6.1$ $(\mathrm{m}, 1 \mathrm{H}), 6.86(\mathrm{~d}, J=10.0 \mathrm{~Hz}, 1 \mathrm{H}) \mathrm{ppm} ;{ }^{13} \mathrm{C} \mathrm{NMR}\left(\mathrm{CDCl}_{3}, 75 \mathrm{MHz}, \mathrm{ppm}\right) \delta 18.5188 .15,128.41,133.99$, $136.89 \mathrm{ppm}$.

(E)-1,1-Dibromo-4-(2-furyl)-1,3-butadiene. The title compound was prepared according to preceding Procedure: Yield 74\%; ${ }^{1} \mathrm{H}$ NMR $\left(\mathrm{CDCl}_{3}, 300 \mathrm{MHz}\right) \delta$ 6.35-6.4 (m, 2H), $6.43(\mathrm{~d}, J=15.5 \mathrm{~Hz}, 1 \mathrm{H}), 6.63(\mathrm{dd}$, $J=10.5$ and $15.5 \mathrm{~Hz}, 1 \mathrm{H}), 6.98(\mathrm{~d}, J=10.5 \mathrm{~Hz}, 1 \mathrm{H}), 7.39(\mathrm{~s}, 1 \mathrm{H}) \mathrm{ppm} ;{ }^{13} \mathrm{C} \mathrm{NMR}\left(\mathrm{CDCl}_{3}, 75 \mathrm{MHz}\right) \delta 91.28$, $110.77,111.95,122.45,123.40,136.66,143.08,152.15$ ppm; HRMS m/z calcd for $\mathrm{C}_{8} \mathrm{H}_{6} \mathrm{Br}_{2} \mathrm{O}: 275.8785$ found: 275.8786 .

1,1-Dibromo-4-phenyl-1-buten-3-yne. ${ }^{12}$ To a solution of phenylacetylene $(3.06 \mathrm{~g}, 30 \mathrm{mmol})$ in dry THF $(50 \mathrm{~mL})$ was added ${ }^{n} \mathrm{BuLi}(2.5 \mathrm{M}$ in hexane, $12 \mathrm{~mL}, 30 \mathrm{mmol})$ at $-78^{\circ} \mathrm{C}$. After $30 \mathrm{~min}$, dry DMF $(6.6 \mathrm{~g}$, $90 \mathrm{mmol}$ ) was added at $-78^{\circ} \mathrm{C}$, and the reaction mixture was warm to $23^{\circ} \mathrm{C}$. After $1 \mathrm{~h}$ of stirring, the mixture was poured into a vigorously stirred biphasic solution prepared from a $10 \%$ aqueous solution of $\mathrm{KH}_{2} \mathrm{PO}_{4}$ and ether cooled at $0{ }^{\circ} \mathrm{C}$. The organic extract was washed with water, dried over $\mathrm{MgSO}_{4}$, filtered, and

\footnotetext{
${ }^{11}$ Baily, L.; Waldmann, H. Chem. Eur. J. 2004, 10, 2759.

${ }^{12}$ Kuroda, H.; Hanaki, E.; Izawa, H.; Kano, M.; Itahashi, H. Tetrahedron 2004, 60, 1913. Uenishi, J.; Kawahama, R.; Yonemitsu, O. J. Org. Chem. 1996, 61, 5716.
} 
concentrated. In another flask, a mixture of tetrabromomethane $(16.6 \mathrm{~g}, 50 \mathrm{mmol})$ and $\mathrm{PPh}_{3}(26.2 \mathrm{~g}, 100$ $\mathrm{mmol})$ in $\mathrm{CH}_{2} \mathrm{Cl}_{2}(150 \mathrm{~mL})$ was stirred at $0{ }^{\circ} \mathrm{C}$ for $20 \mathrm{~min}$. To this were added consecutively 2,6-lutidine (6.7 $\mathrm{g}, 62.5 \mathrm{mmol})$ and a solution of 3-phenylpropiolaldehyde generated above $(3.2 \mathrm{~g})$ in $\mathrm{CH}_{2} \mathrm{Cl}_{2}$ at $0{ }^{\circ} \mathrm{C}$. The reaction mixture was stirred for $30 \mathrm{~min}$ at $0{ }^{\circ} \mathrm{C}$ and poured into pentane. After filtration through a layer of silica gel and concentration, purification by column chromatography (silica gel, pentane) afforded $6.86 \mathrm{~g}$ (79\% from phenylacetylene) of the title compound: yellow oil; ${ }^{1} \mathrm{H} \mathrm{NMR}\left(\mathrm{CDCl}_{3}, 300 \mathrm{MHz}\right) \delta 6.79(\mathrm{~s}, 1 \mathrm{H})$, 7.3-7.4 (m, 3H), 7.5-7.55 (m, 2H) ppm; ${ }^{13} \mathrm{C} \mathrm{NMR}\left(\mathrm{CDCl}_{3}, 75 \mathrm{MHz}\right) \delta 86.17,97.13,101.81,119.54,122.31$, $128.35,128.95,131.47 \mathrm{ppm}$.

1,1-Dibromo-4-(p-tolyl)-1-buten-3-yne: The title compound was prepared according to the preceding procedure from $p$-tolylacetylene: Yield $75 \%$; mp 59-60 ${ }^{\circ} \mathrm{C}$; yellow solid; ${ }^{1} \mathrm{H} \mathrm{NMR}\left(\mathrm{CDCl}_{3}, 300 \mathrm{MHz}\right) \delta 2.32$ $(\mathrm{s}, 3 \mathrm{H}), 6.71(\mathrm{~s}, 1 \mathrm{H}), 7.10(\mathrm{~d}, J=7.8 \mathrm{~Hz}, 2 \mathrm{H}), 7.35(\mathrm{~d}, J=7.8 \mathrm{~Hz}, 2 \mathrm{H}) \mathrm{ppm} ;{ }^{13} \mathrm{C} \mathrm{NMR}\left(\mathrm{CDCl}_{3}, 75 \mathrm{MHz}\right) \delta$ 21.56, 85.65, 97.47, 101.20, 119.28, 119.70, 129.16 (2C), 131.41 (2C), 139.24 ppm; IR $\left(\mathrm{CCl}_{4}\right)$ 3077, 3015, 2975, 2939, 2203, 1506, $1445 \mathrm{~cm}^{-1}$; HRMS m/z calcd for $\mathrm{C}_{11} \mathrm{H}_{8} \mathrm{Br}_{2}: 297.8993$; found: 297.8991.

(Z)-1-(tert-Butyldimethylsilyloxy)-4-bromo-7-phenyl-4-heptene-2,6-diyne: $\quad$ To a solution of 1-(tert-butyldimethylsilyloxy)-2-propyne (408 mg, $2.4 \mathrm{mmol})$ in THF $(4 \mathrm{~mL})$ was added ${ }^{n} \mathrm{BuLi}(2.5 \mathrm{M}$ in hexane, $0.96 \mathrm{~mL}, 2.4 \mathrm{mmol}$ ) at $-78{ }^{\circ} \mathrm{C}$ for $30 \mathrm{~min}$ and treated with anhydrous $\mathrm{ZnBr}_{2}$ (324 mg, $1.44 \mathrm{mmol}$ ) in THF $(3 \mathrm{~mL})$. The mixture thus obtained was stirred for $5 \mathrm{~min}$ at $-78{ }^{\circ} \mathrm{C}$, warmed to $0{ }^{\circ} \mathrm{C}$ over $25 \mathrm{~min}$, and added via cannula to a mixture of 1,1-dibromo-4-phenyl-1-en-3-butyne (572 $\mathrm{mg}, 2 \mathrm{mmol}$ ) and $\mathrm{Pd}(\mathrm{DPEphos}) \mathrm{Cl}_{2}(71.5 \mathrm{mg}, 0.1 \mathrm{mmol})$ in THF $(5 \mathrm{~mL})$ at $0{ }^{\circ} \mathrm{C}$. After $8 \mathrm{~h} \mathrm{GLC}$ analysis indicated that the starting material had been completely consumed. The reaction mixture was quenched with aqueous $\mathrm{NH}_{4} \mathrm{Cl}$, extracted with ether, washed successively with saturated $\mathrm{NaHCO}_{3}$ and brine, dried over $\mathrm{MgSO}_{4}$, filtered, and concentrated. Purification by column chromatography (silica gel, pentane) afforded $532 \mathrm{mg}$ (71\%) of the title compound: colorless oil; ${ }^{1} \mathrm{H} \mathrm{NMR}\left(\mathrm{CDCl}_{3}, 300 \mathrm{MHz}\right) \delta 0.16(\mathrm{~s}, 6 \mathrm{H}), 0.94(\mathrm{~s}, 9 \mathrm{H}), 4.52(\mathrm{~s}, 2 \mathrm{H})$,

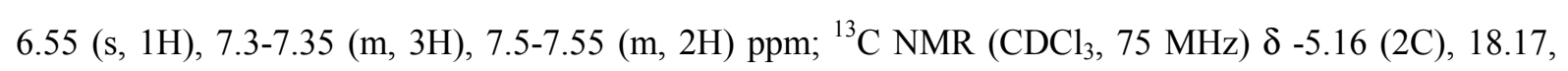
25.73 (3C), 51.96, 83.31, 86.73, 94.53, 101.03, 110.35, 119.21, 122.49, 128.35 (2C), 129.01, 131.62 (2C) ppm; IR (neat) 2955, 2929, 2857, 2216, $2192 \mathrm{~cm}^{-1}$; HRMS m/z calcd for $\mathrm{C}_{19} \mathrm{H}_{23} \mathrm{BrOSi}$ : 374.0702; found: 374.0703. 
7-Phenyl-2,4,6-heptatriyn-1-ol (10). ${ }^{13}$ To a solution of (Z)-1-(tert-butyldimethylsilyloxy) -4-bromo-7- phenyl-4-heptene-2,6-diyne $(532 \mathrm{mg}, 1.4 \mathrm{mmol})$ in methanol $(5 \mathrm{~mL})$ was added $\mathrm{K}_{2} \mathrm{CO}_{3}(580$ $\mathrm{mg}, 4.2 \mathrm{mmol}$ ) at $23^{\circ} \mathrm{C}$. After $12 \mathrm{~h}$, the reaction mixture was quenched with water, extracted with ethyl acetate, washed with brine, dried over $\mathrm{MgSO}_{4}$, filtered, and concentrated. Purification by column chromatography (silica gel, 1/4 ethyl acetate-hexane) afforded $204 \mathrm{mg}(81 \%)$ of the title compound: colorless oil; ${ }^{1} \mathrm{H}$ NMR $\left(\mathrm{CDCl}_{3}, 300 \mathrm{MHz}\right) \delta 2.12(\mathrm{~s}, 1 \mathrm{H}), 4.39(\mathrm{~s}, 2 \mathrm{H})$, 7.3-7.4 (m, 3H), 7.5-7.55 (m, 2H) ppm; ${ }^{13} \mathrm{C}$ NMR $\left(\mathrm{CDCl}_{3}, 75 \mathrm{MHz}\right): \delta$ 51.43, 63.38, 65.81, 70.71, 74.09, 77.28, 77.91, 120.61, 128.43, $129.74,132.97 \mathrm{ppm}$.

\section{Representative Procedure for the Convergent Synthesis: (E)-11-Phenyl-2-undecene-} 4,6,8,10-tetrayne (13). To a solution of ( $1 E, 5 E)$-1-chloro-1,5-heptadien-3-yne (278 mg, $2.2 \mathrm{mmol})$ in THF $(6 \mathrm{~mL})$ was added ${ }^{n} \mathrm{BuLi}(2.5 \mathrm{M}$ in hexane, $1.76 \mathrm{~mL}, 4.4 \mathrm{mmol})$ at $-78^{\circ} \mathrm{C}$ for $1 \mathrm{~h}$ and treated with anhydrous $\mathrm{ZnBr}_{2}(283 \mathrm{mg}, 1.26 \mathrm{mmol})$ in THF $(3 \mathrm{~mL})$. The mixture thus obtained was stirred for $5 \mathrm{~min}$ at $-78{ }^{\circ} \mathrm{C}$ and warmed to $0{ }^{\circ} \mathrm{C}$ over $25 \mathrm{~min}$. 1,1-Dibromo-4-phenyl-1-buten-3-yne (572 mg, $\left.2 \mathrm{mmol}\right)$ and $\mathrm{Pd}\left(\mathrm{PPh}_{3}\right)_{4}(213$ $\mathrm{mg}, 0.18 \mathrm{mmol})$ in THF $(10 \mathrm{~mL})$ and $\mathrm{NEt}_{3}(0.28 \mathrm{~mL}, 2 \mathrm{mmol})$ were successively added to the reaction mixture at $0{ }^{\circ} \mathrm{C}$. The reaction mixture was stirred at $23{ }^{\circ} \mathrm{C}$ for $12 \mathrm{~h}$, quenched with aqueous $\mathrm{NH}_{4} \mathrm{Cl}$, extracted with ether, washed successively with saturated $\mathrm{NaHCO}_{3}$ and brine, dried over $\mathrm{MgSO}_{4}$, filtered, and concentrated. Purification by column chromatography (silica gel, pentane) afforded $280 \mathrm{mg}$ (66\%) of the title compound: $\mathrm{mp} 82-83{ }^{\circ} \mathrm{C}$; yellow solid; ${ }^{1} \mathrm{H} \mathrm{NMR}\left(\mathrm{CDCl}_{3}, 400 \mathrm{MHz}\right) \delta 1.85(\mathrm{dd}, J=6.8$ and $2.0 \mathrm{~Hz}, 3 \mathrm{H})$. $5.46(\mathrm{dd}, J=16.0$ and $2.0 \mathrm{~Hz}, 1 \mathrm{H}), 6.45(\mathrm{dq}, J=16.0$ and $6.8 \mathrm{~Hz}, 1 \mathrm{H}), 7.3-7.4(\mathrm{~m}, 5 \mathrm{H}) \mathrm{ppm} ;{ }^{13} \mathrm{C}$ NMR $\left(\mathrm{CDCl}_{3}, 100 \mathrm{MHz}\right) \delta 19.16,63.11,63.98,66.14,67.31,72.87,74.43,77.10,77.37,108.98,120.47,128.49$ (2C), 129.89, 133.12 (2C), 147.06 ppm; IR $\left(\mathrm{CCl}_{4}\right): 2983,2937,2196,1490,1442 \mathrm{~cm}^{-1}$; HRMS m/z calcd for $\mathrm{C}_{17} \mathrm{H}_{10}$ : 214.0783; found: 214.0783 .

1-Phenyl-8-(p-tolyl)-1,3,5,7-octatetrayne (14). ${ }^{8}$ The title compound was prepared according to the preceding procedure: Yield 68\%; mp 93-94 ${ }^{\circ} \mathrm{C}$; yellow solid; ${ }^{1} \mathrm{H}$ NMR $\left(\mathrm{CDCl}_{3}, 300 \mathrm{MHz}\right) \delta 2.33(\mathrm{~s}, 3 \mathrm{H})$, 7.05-7.1 (m, 2H), 7.3-7.4 (m, 5H),7.45-7.5 (m, 2H) ppm; ${ }^{13} \mathrm{C} \mathrm{NMR}\left(\mathrm{CDCl}_{3}, 75 \mathrm{MHz}\right) \delta$ 21.67, 63.53, 63.92, $66.92,67.29,73.91,74.45,77.62,78.15,117.17,120.40,128.46$ (2C), 129.300 (2C), 129.89, 133.09 (4C), $140.59 \mathrm{ppm}$.

\footnotetext{
${ }^{13}$ Luu, T.; Shi, W.; Lowary, T. L.; Tykwinski, R. R. Synthesis, 2005, 3167.
} 
1-Phenyl-1,3,5,7,9-undecapentayne (15a). The title compound was prepared according to the above-described the procedure for the synthesis of 13: Yield $43 \%$; decomposition at $75{ }^{\circ} \mathrm{C}$; yellow solid; ${ }^{1} \mathrm{H}$ $\mathrm{NMR}\left(\mathrm{CDCl}_{3}, 400 \mathrm{MHz}\right) \delta 2.01(\mathrm{~s}, 3 \mathrm{H}), 7.25-7.4(\mathrm{~m}, 3 \mathrm{H}), 7.5-7.55(\mathrm{~m}, 2 \mathrm{H}) \mathrm{ppm} ;{ }^{13} \mathrm{C} \mathrm{NMR}\left(\mathrm{CDCl}_{3}, 100\right.$ MHz) $\delta 4.74,59.73,60.93(2 \mathrm{C}), 63.91,64.92(2 \mathrm{C}), 67.42,74.39,76.51,77.81,120.28,128.56(2 \mathrm{C}), 130.11$, 133.32 (2C) ppm; IR $\left(\mathrm{CCl}_{4}\right) 3070,3040,2212,2188,1495,1441 \mathrm{~cm}^{-1} ;$ HRMS m/z calcd for $\mathrm{C}_{17} \mathrm{H}_{8}$ : 212.0626; found: 212.0627 .

(E)-14-Phenyl-2-tridecene-4,6,8,10,12-pentayne (15b): The title compound was prepared according to the above-described the procedure for the synthesis of 13 : Yield $48 \%$; decomposition started at $60{ }^{\circ} \mathrm{C}$; yellow solid; ${ }^{1} \mathrm{H}$ NMR $\left(\mathrm{CDCl}_{3}, 400 \mathrm{MHz}\right) \delta 1.85(\mathrm{dd}, J=6.8$ and $1.6 \mathrm{~Hz}, 3 \mathrm{H}) 5.56(\mathrm{dd}, J=16.0$ and $1.6 \mathrm{~Hz}$, $1 \mathrm{H}), 6.50(\mathrm{dq}, J=16.0$ and $6.8 \mathrm{~Hz}, 1 \mathrm{H}), 7.2-7.4(\mathrm{~m}, 3 \mathrm{H}), 7.5-7.55(\mathrm{~m}, 2 \mathrm{H}) \mathrm{ppm} ;{ }^{13} \mathrm{C} \mathrm{NMR}\left(\mathrm{CDCl}_{3}, 100 \mathrm{MHz}\right)$ $\delta 19.24,62.43,63.09,63.93,64.60,66.26,67.32,72.85,74.39,76.83,77.24,108.98,120.28,128.56(2 \mathrm{C})$, 130.13, 133.31 (2C), 147.71 ppm; IR $\left(\mathrm{CCl}_{4}\right)$ : 2918, 2178, 1495, $1441 \mathrm{~cm}^{-1}$; HRMS m/z calcd for $\mathrm{C}_{19} \mathrm{H}_{10}$ : 238.0783; found: 238.0780 .

1-Phenyl-10-(p-tolyl)-1,3,5,7,9-decapentayne (15c). The title compound was prepared according to the above-described the procedure for the synthesis of 13 : Yield $55 \%$; decomposition started at $110{ }^{\circ} \mathrm{C}$; yellow solid; ${ }^{1} \mathrm{H}$ NMR $\left(\mathrm{CDCl}_{3}, 400 \mathrm{MHz}\right) \delta 2.36(\mathrm{~s}, 3 \mathrm{H}), 7.1-7.15(\mathrm{~m}, 2 \mathrm{H}), 7.3-7.4(\mathrm{~m}, 5 \mathrm{H})$, 7.5-7.55 (m, 2H) ppm; ${ }^{13} \mathrm{C}$ NMR $\left(\mathrm{CDCl}_{3}, 100 \mathrm{MHz}\right) \delta 21.76,62.65,62.98,64.31,64.55,67.01,67.30,73.88,74.36,77.87$, 117.00, 120.23, 128.55 (2C), 129.38 (2C), 130.14, 133.30 (4C), 140.89 ppm; IR (CCl $): 2918,2842,2174$, $1441 \mathrm{~cm}^{-1}$; HRMS m/z calcd for $\mathrm{C}_{23} \mathrm{H}_{12}$ : 288.0939; found: 288.0939 . 


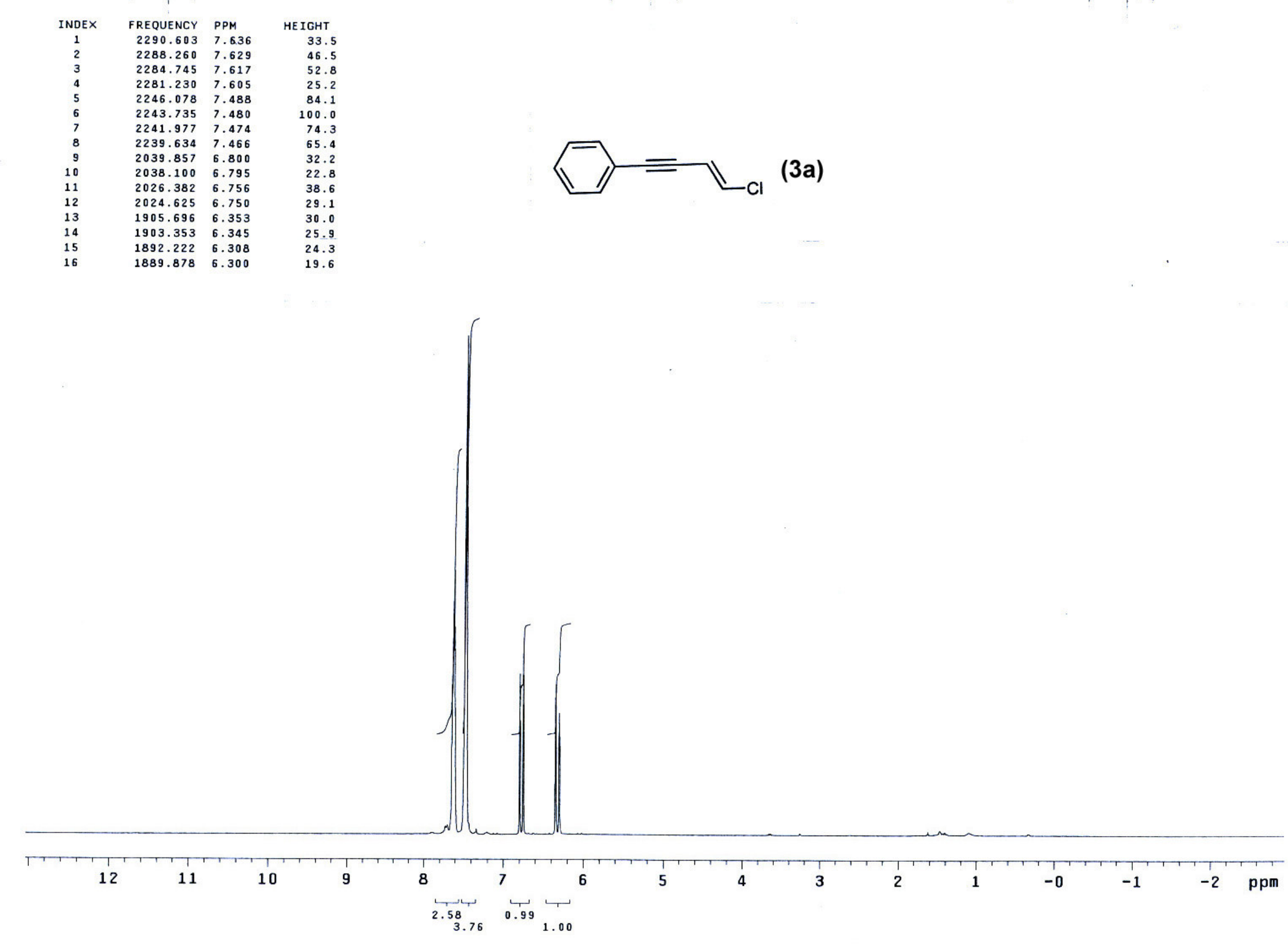




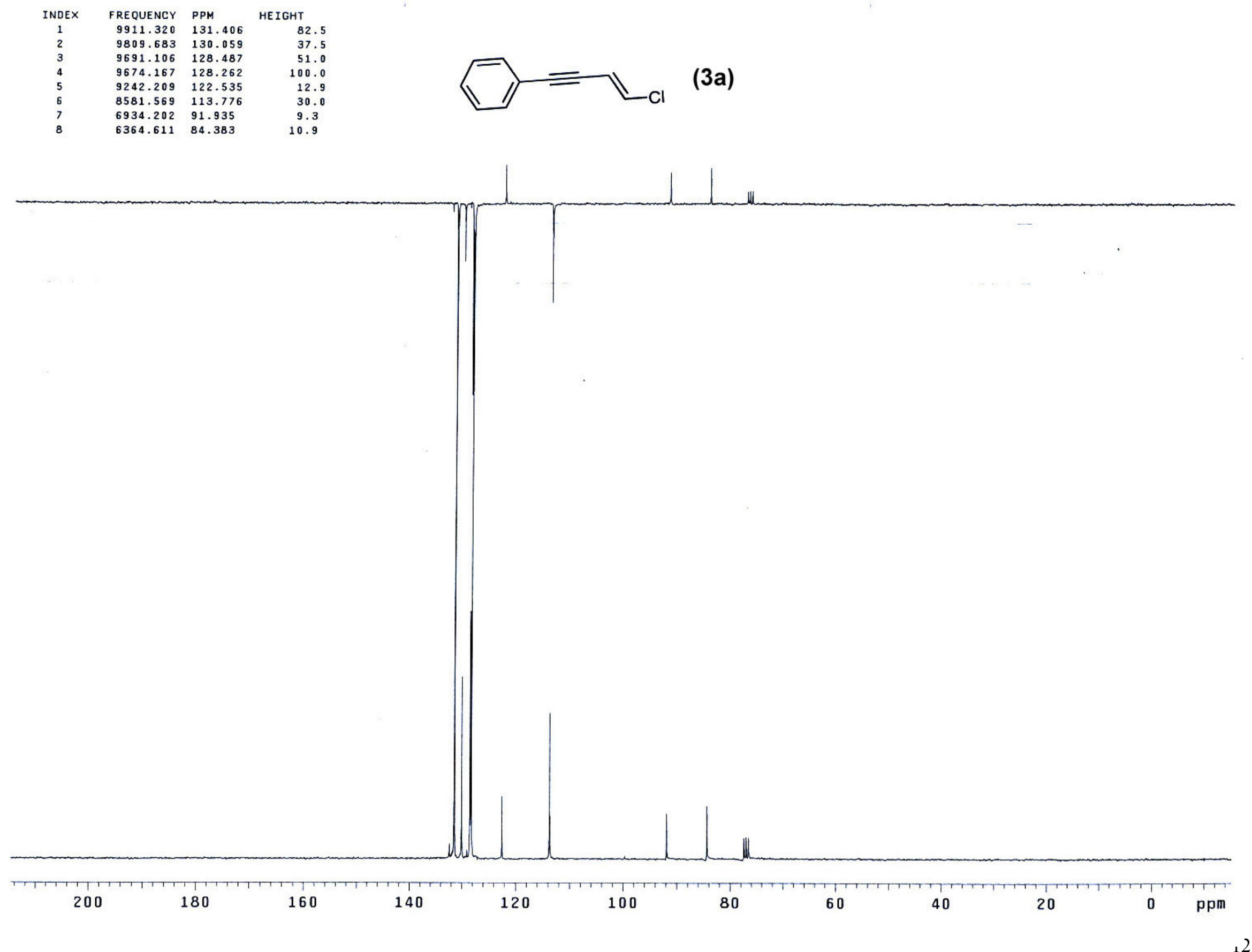




$\begin{array}{crlr}\text { INDEX } & \text { FREQUENCY } & \text { PPM } & \text { HEIGHT } \\ 1 & 1977.381 & 5.592 & 4.2 \\ 2 & 1963.320 & 6.545 & 5.9 \\ 3 & 1786.381 & 5.955 & 6.1 \\ 4 & 1772.905 & 5.910 & 4.7 \\ 5 & 58.004 & 0.193 & 2.9 \\ 6 & 54.488 & 0.182 & 75.0\end{array}$

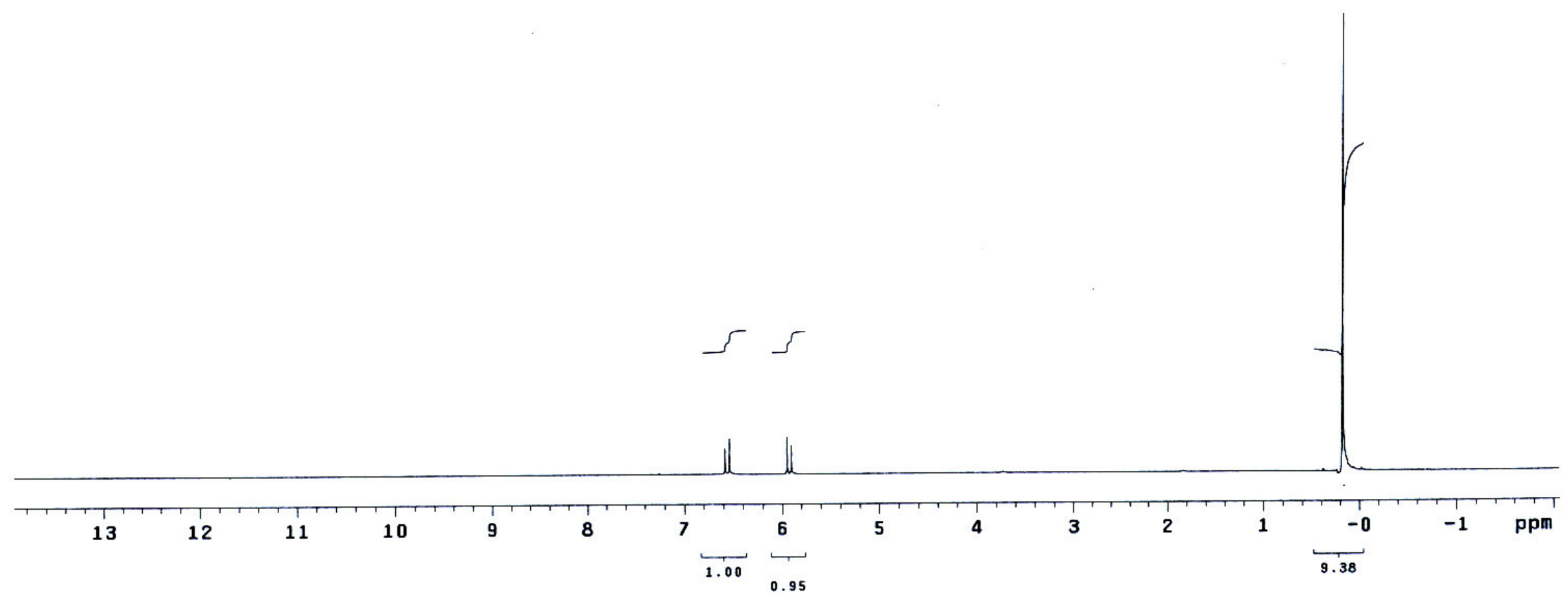



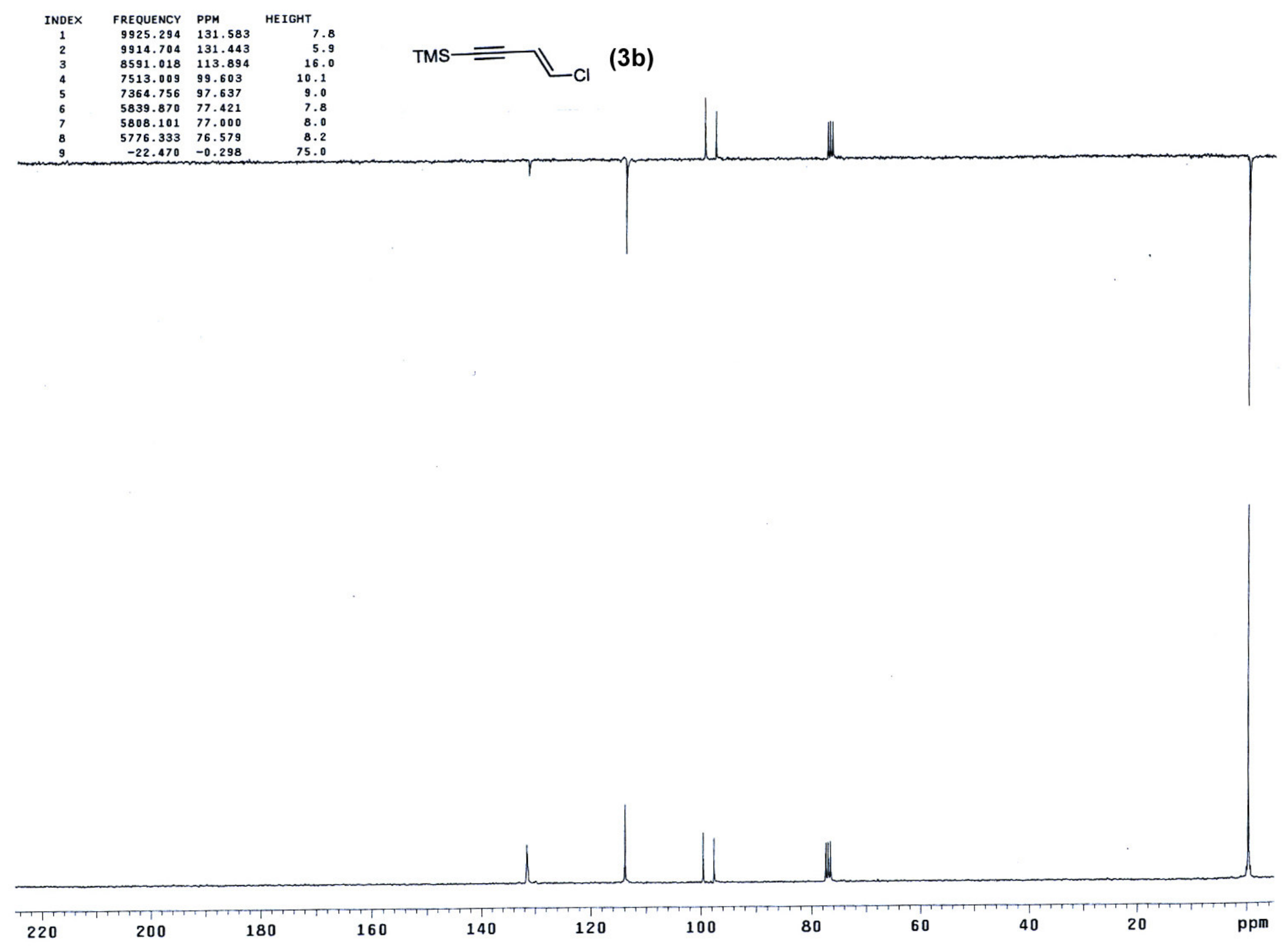


$\begin{array}{crlr}\text { INDEX } & \text { FREQUENCY } & \text { PPM } & \text { HEIGHT } \\ 1 & 1929.808 & 6.434 & 11.3 \\ 2 & 1916.333 & 6.389 & 14.6 \\ 3 & 1769.869 & 5.900 & 4.0 \\ 4 & 1767.526 & 5.893 & 11.4 \\ 5 & 1765.182 & 5.885 & 9.3 \\ 6 & 1756.395 & 5.855 & 3.2 \\ 7 & 1754.051 & 5.848 & 8.1 \\ 8 & 1751.122 & 5.838 & 7.3 \\ 9 & 574.138 & 1.914 & 75.0 \\ 10 & 571.795 & 1.906 & 73.2\end{array}$
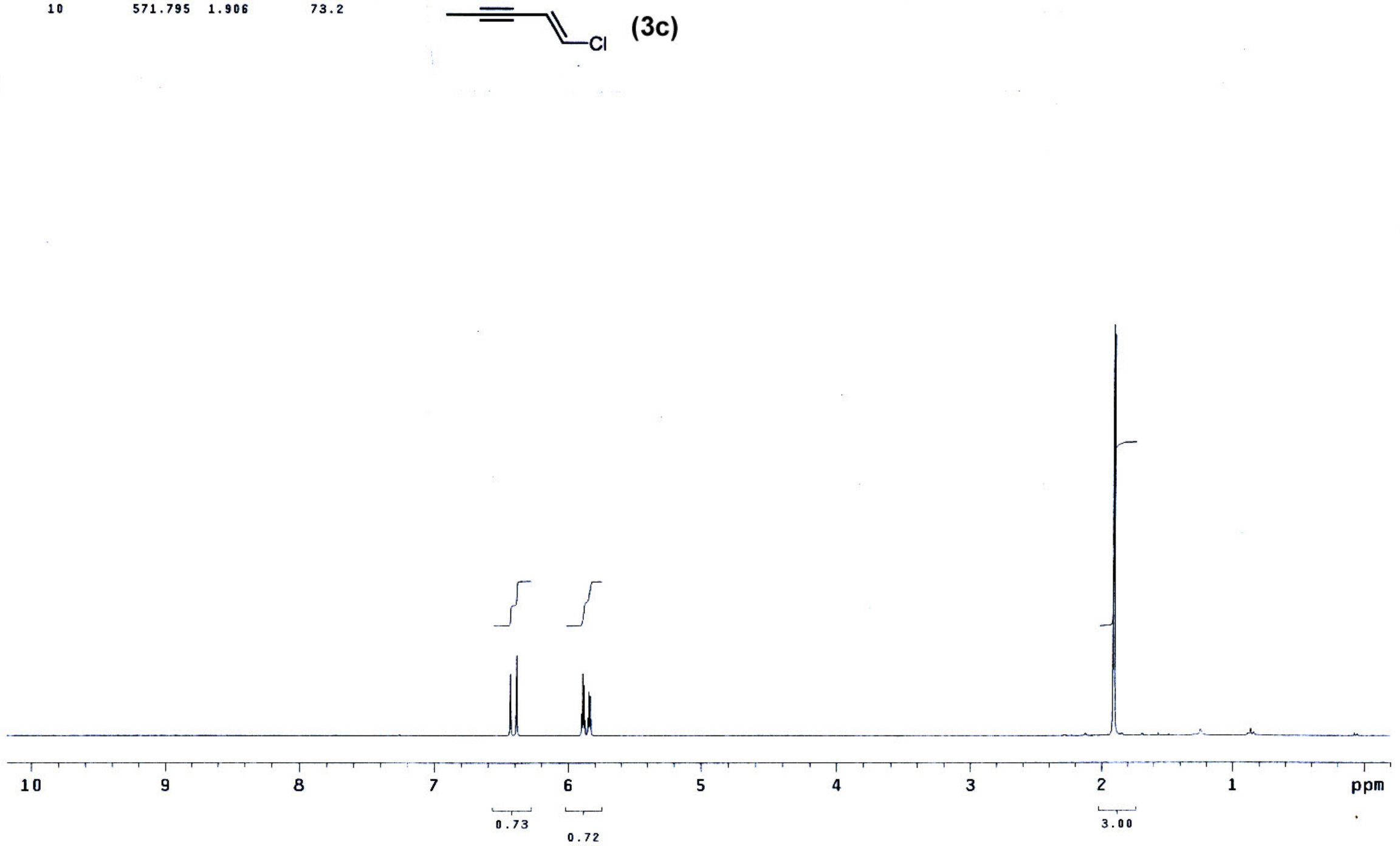

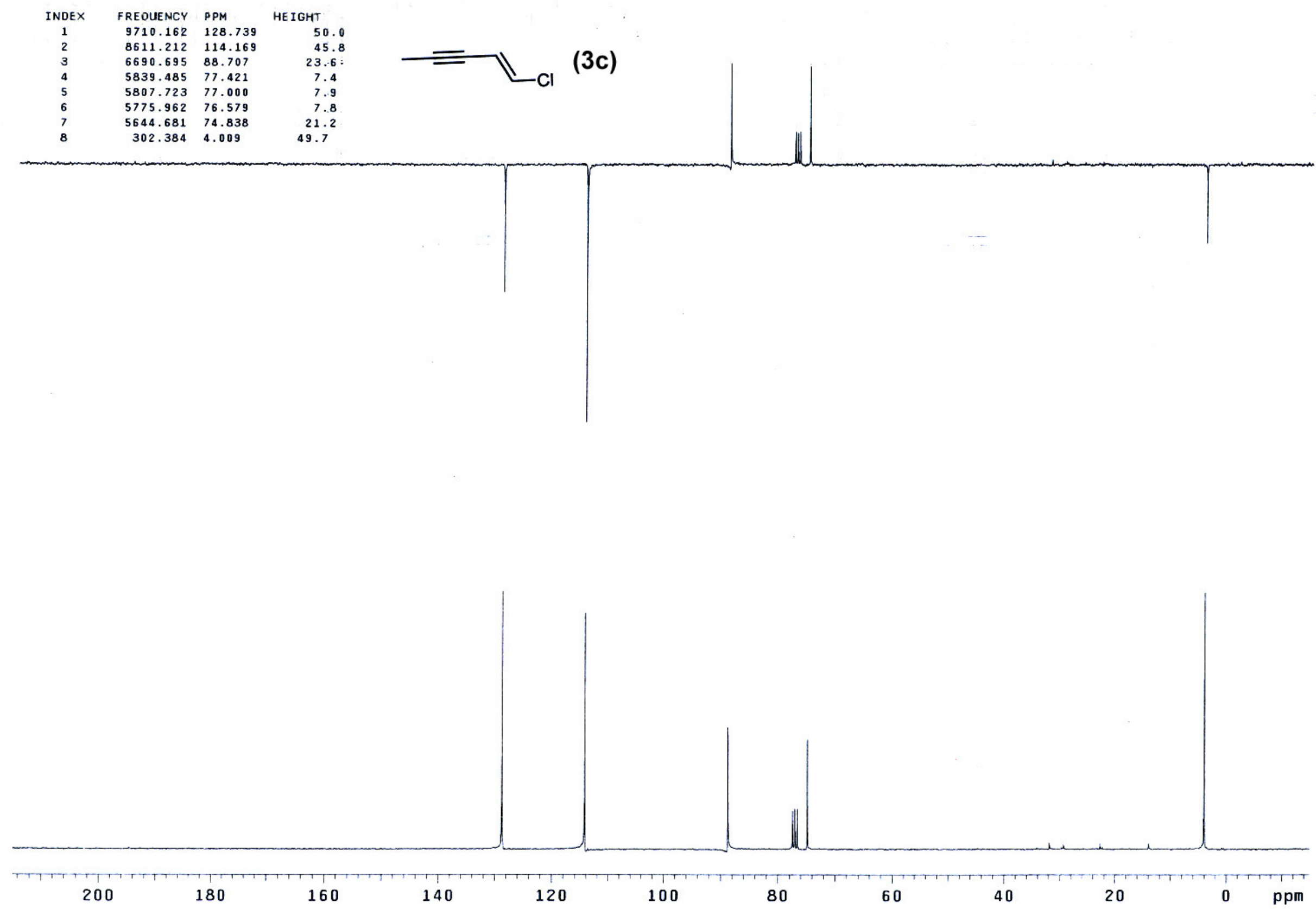


$\begin{array}{crlr}\text { INDEX } & \text { FREQUENCY } & \text { PPM } & \text { HEIGHT } \\ 1 & 2290.603 & 7.636 & 21.7 \\ 2 & 2288.845 & 7.631 & 27.3 \\ 3 & 2282.987 & 7.611 & 31.8 \\ 4 & 2281.229 & 7.605 & 31.4 \\ 5 & 2239.048 & 7.464 & 15.7 \\ 6 & 2237.290 & 7.459 & 28.9 \\ 7 & 2235.532 & 7.453 & 45.0 \\ 8 & 2228.502 & 7.429 & 31.4 \\ 9 & 2048.059 & 6.828 & 25.8 \\ 10 & 2034.584 & 6.783 & 30.7 \\ 11 & 1854.140 & 6.181 & 28.4 \\ 12 & 1840.080 & 6.134 & 24.3\end{array}$
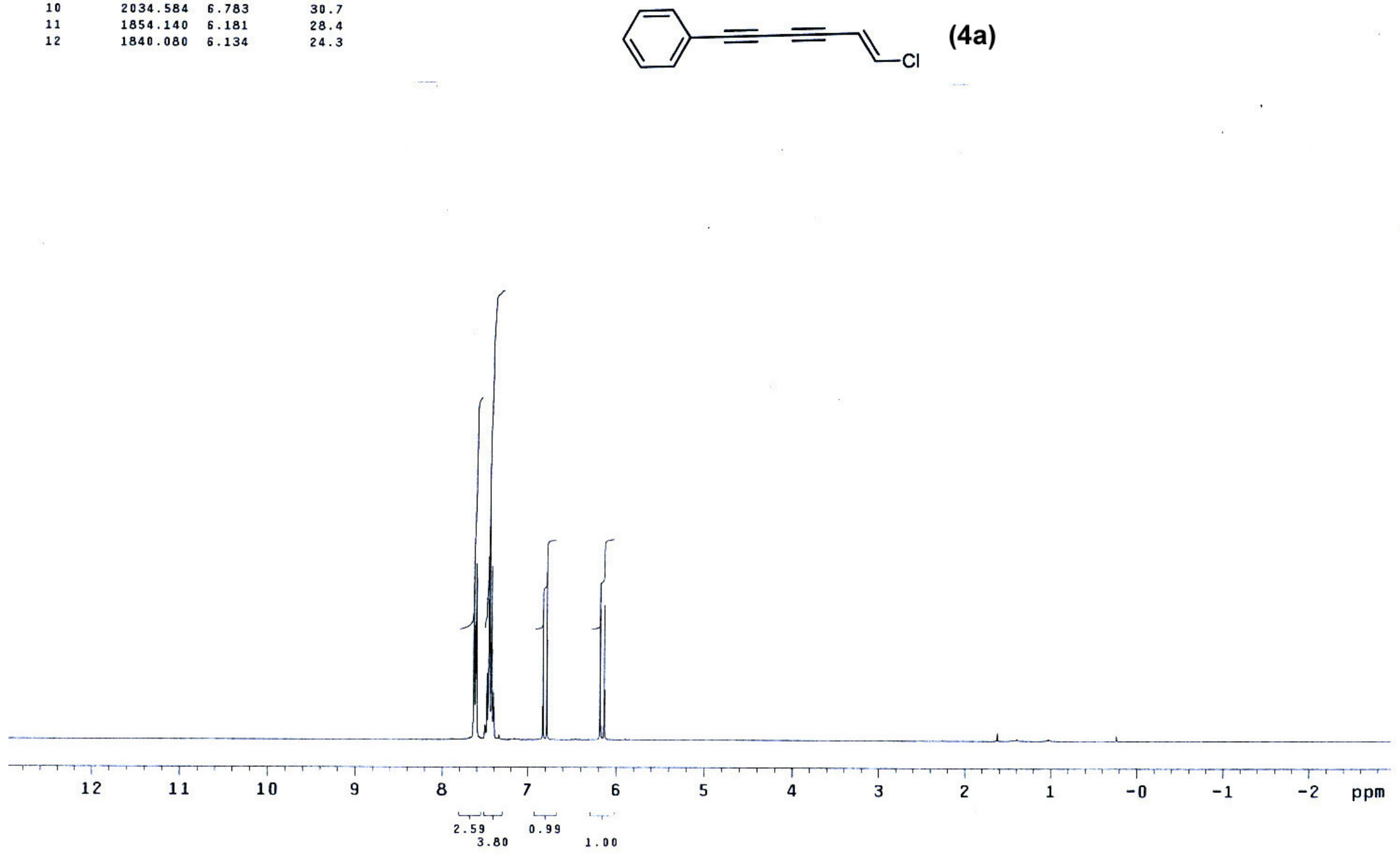


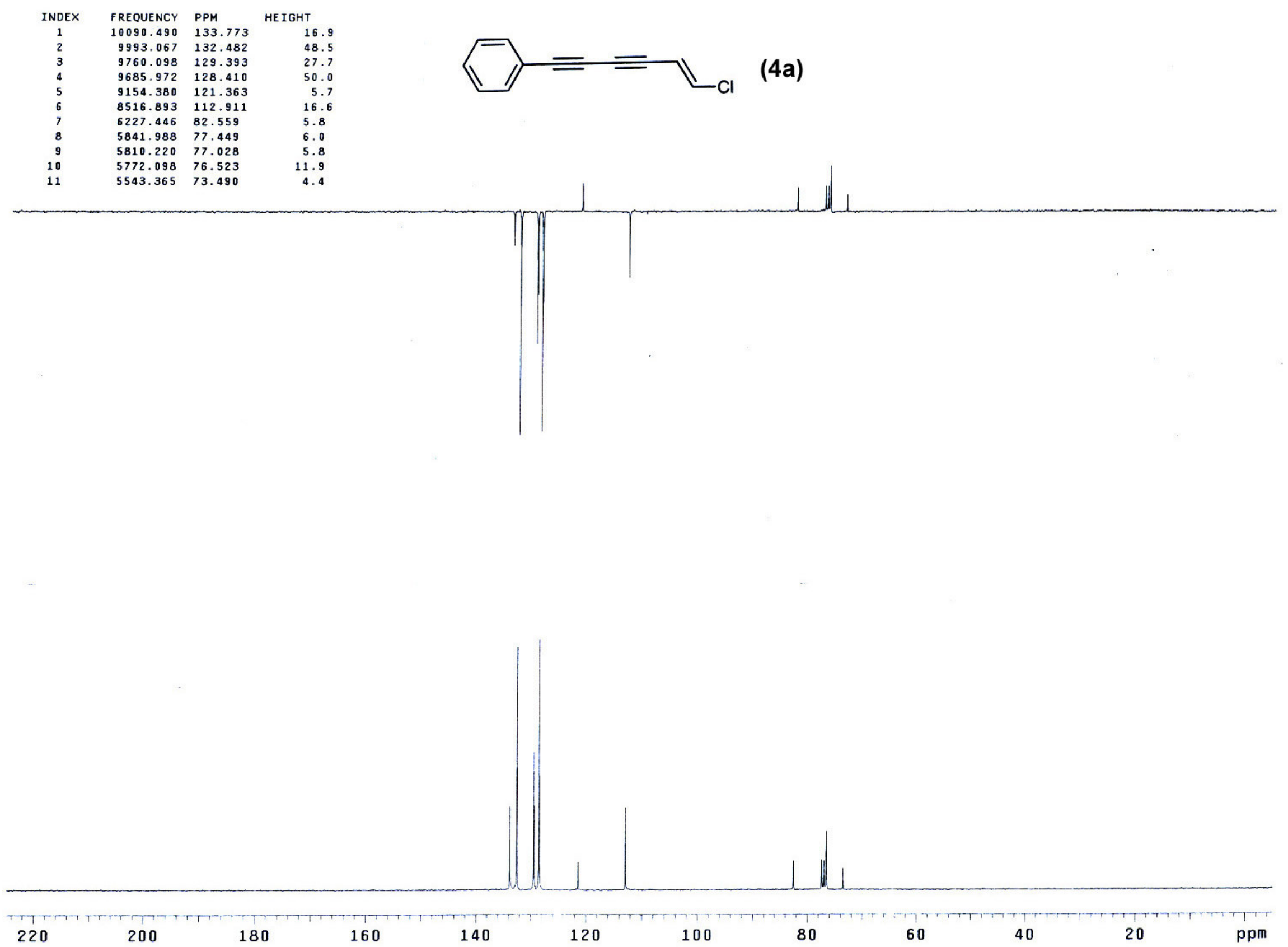




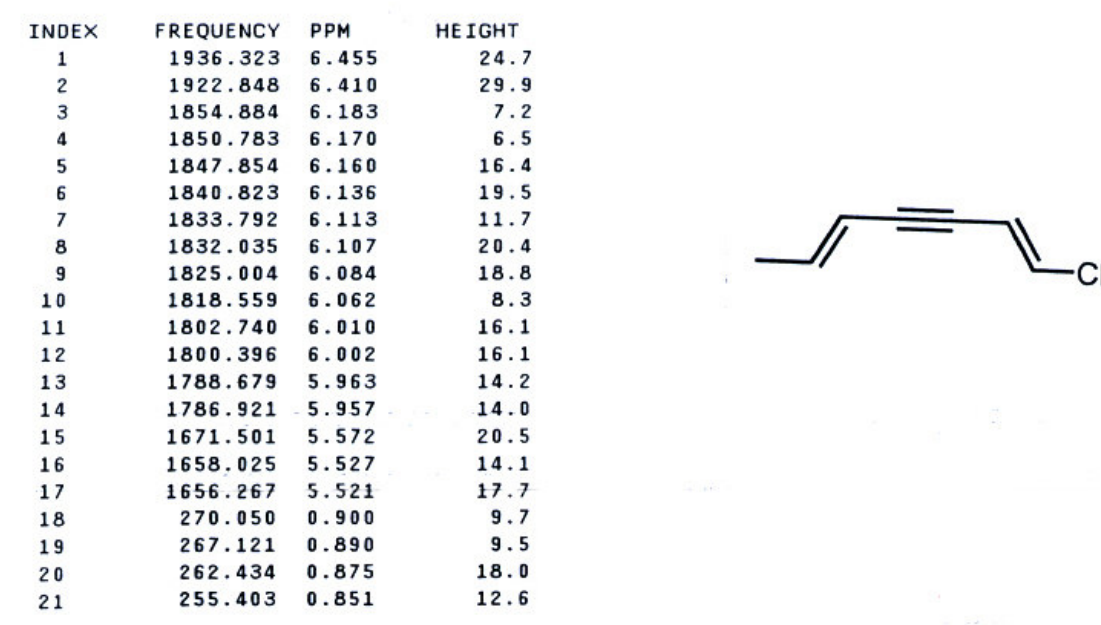

(3d)

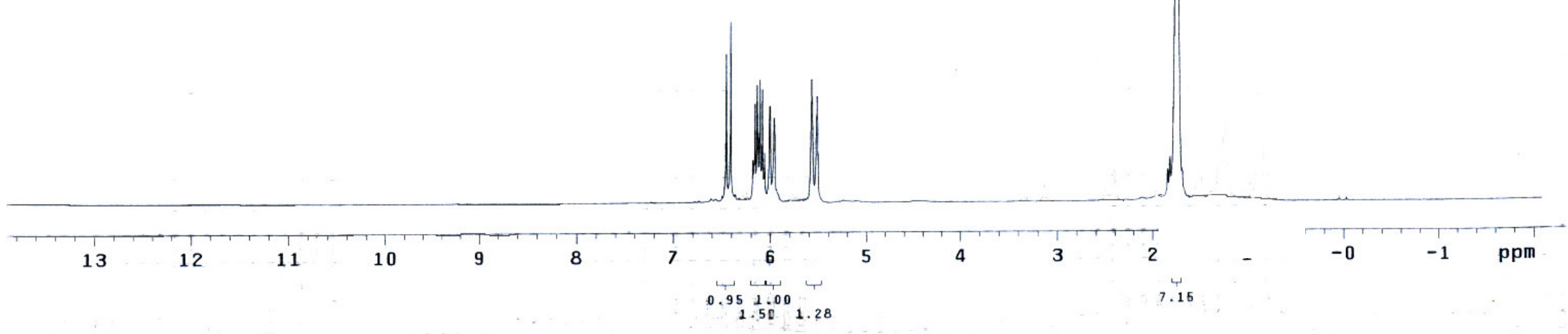



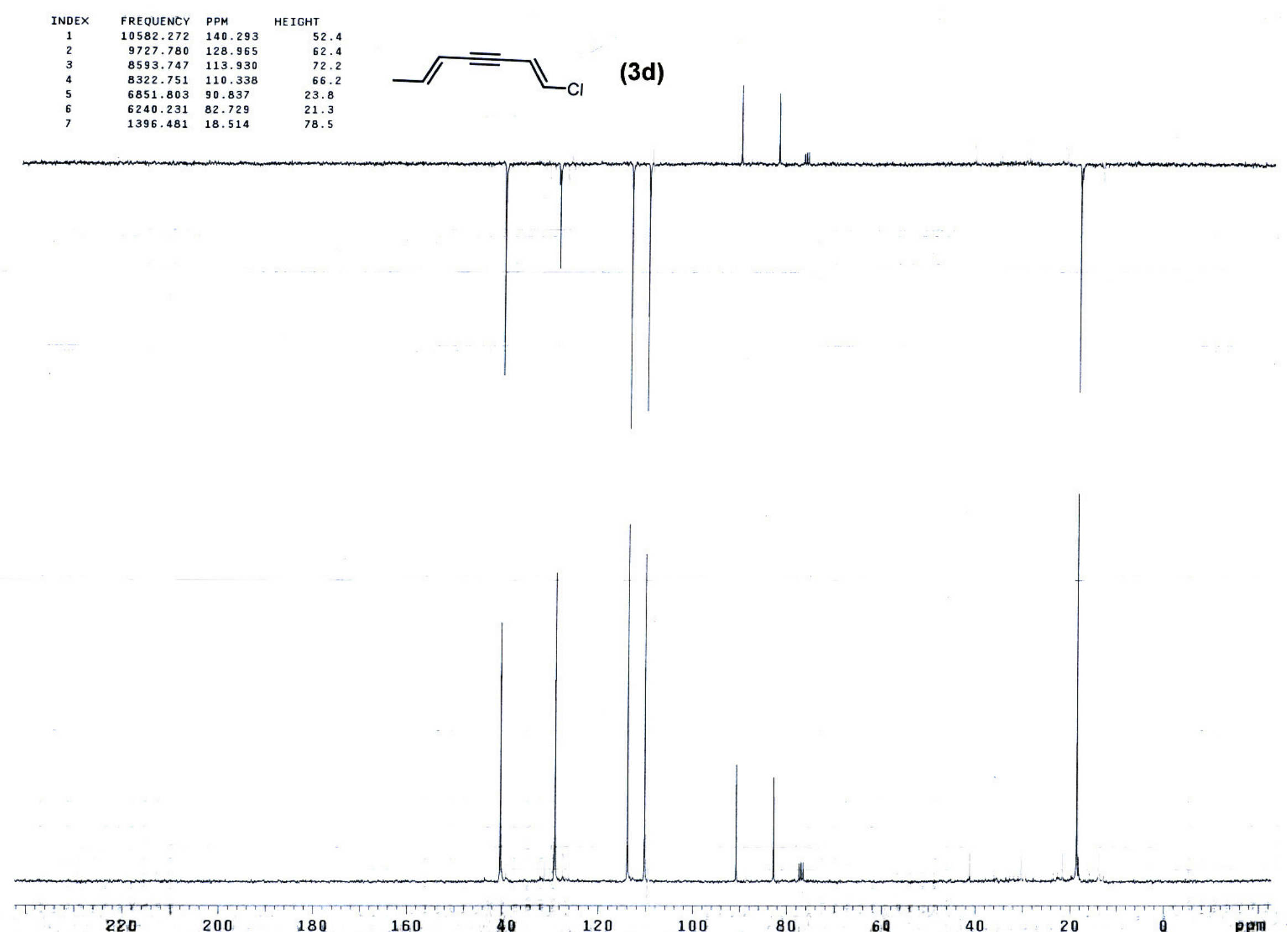

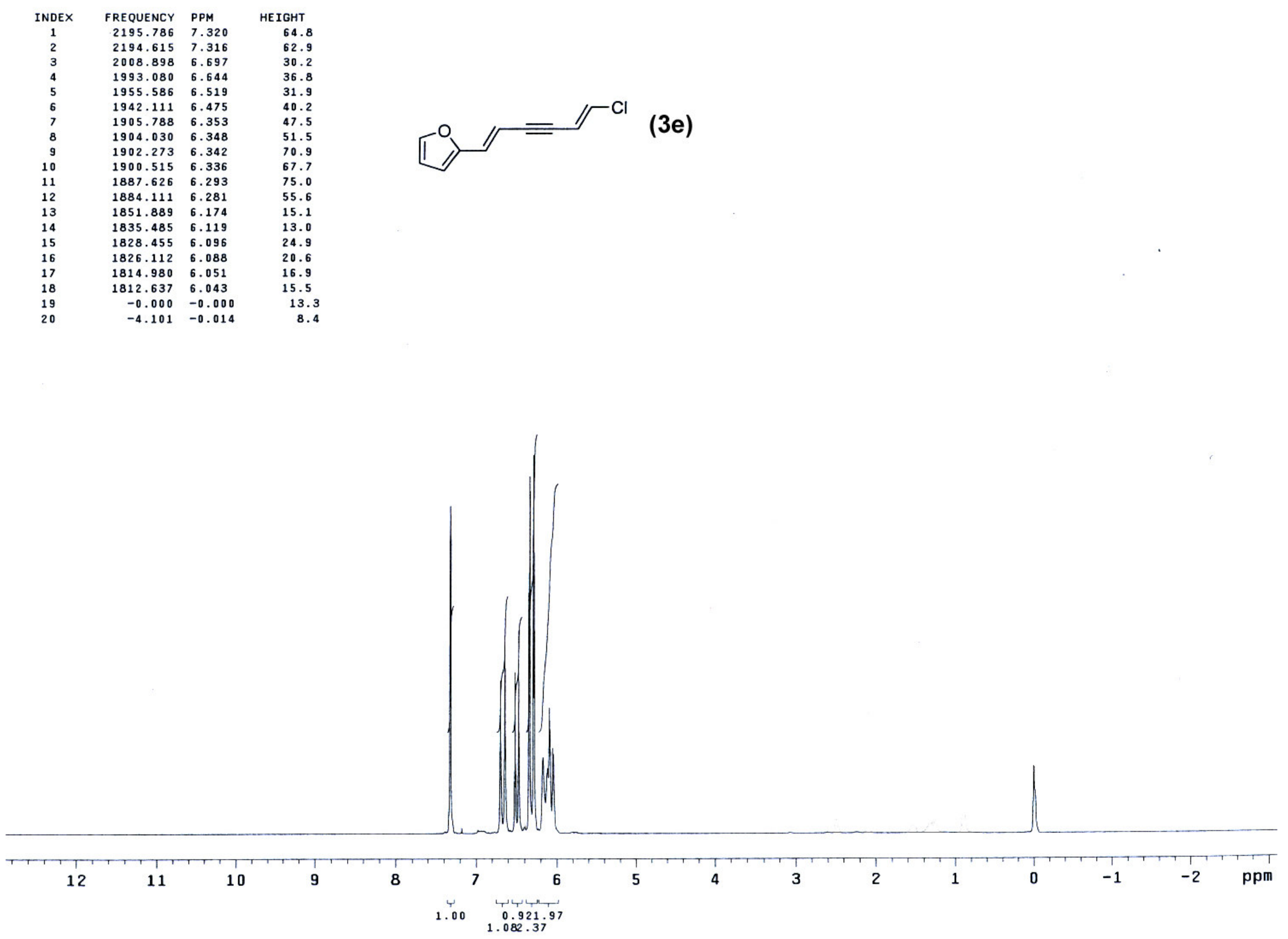


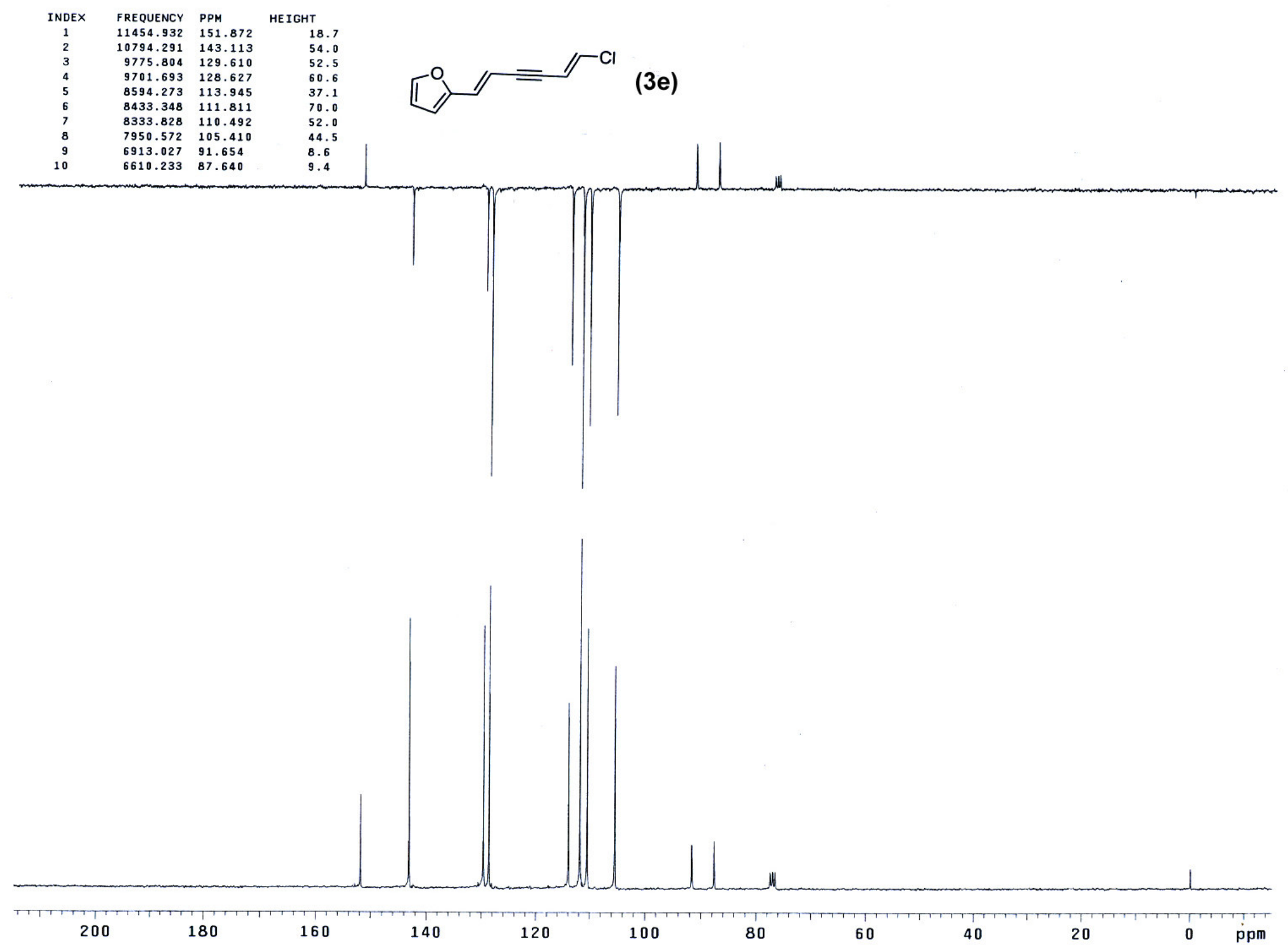




$\begin{array}{crlr}\text { INDEX } & \text { FREQUENCY } & \text { PPM } & \text { HEIGHT } \\ 1 & 2007.811 & 6.694 & 4.6 \\ 2 & 1993.750 & 6.647 & 3.5 \\ 3 & 1791.630 & 5.973 & 4.4 \\ 4 & 1778.155 & 5.928 & 5.0 \\ 5 & 59.841 & 0.199 & 75.0 \\ 6 & 56.326 & 0.188 & 3.1 \\ 7 & 54.569 & 0.182 & 4.0\end{array}$
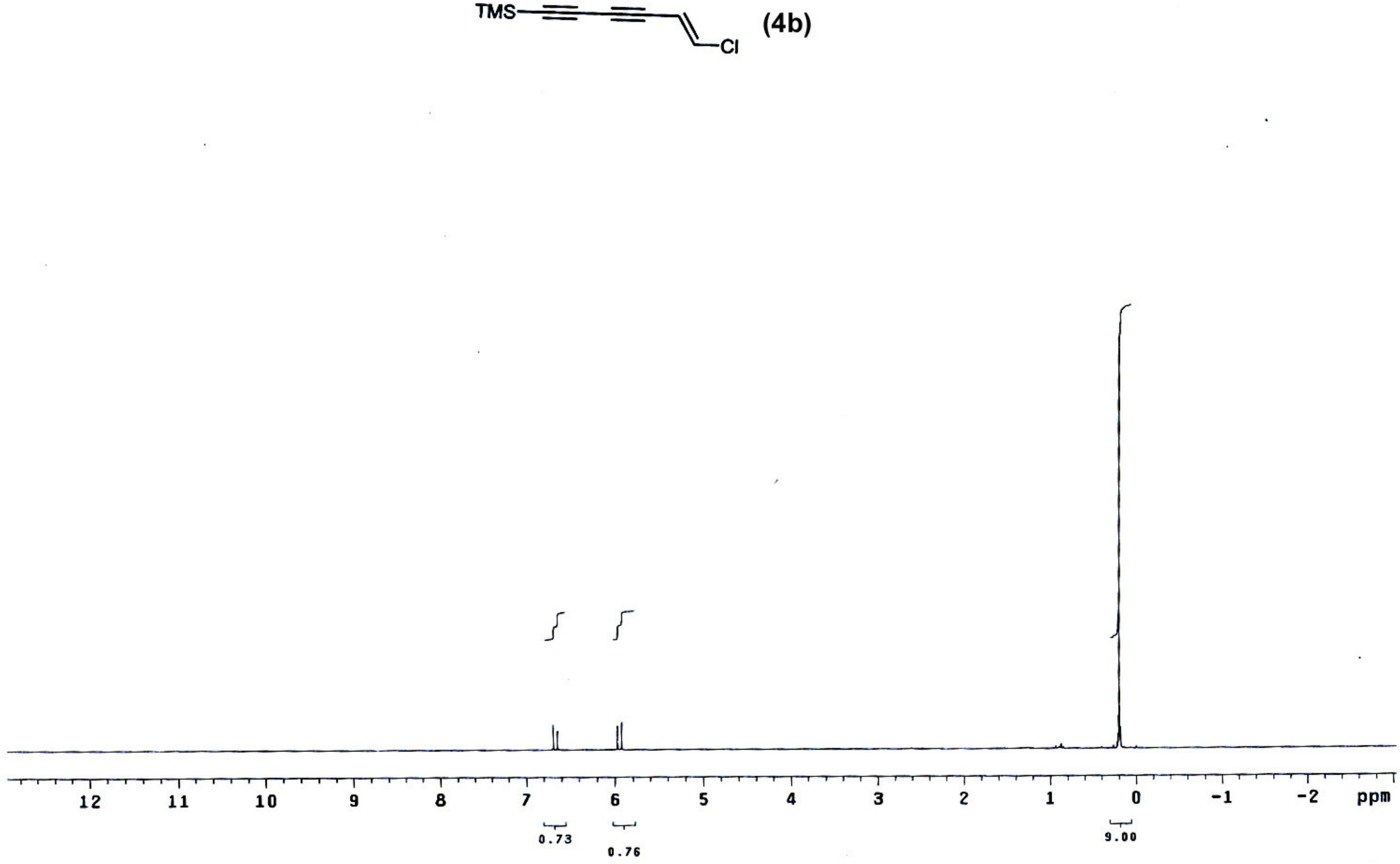

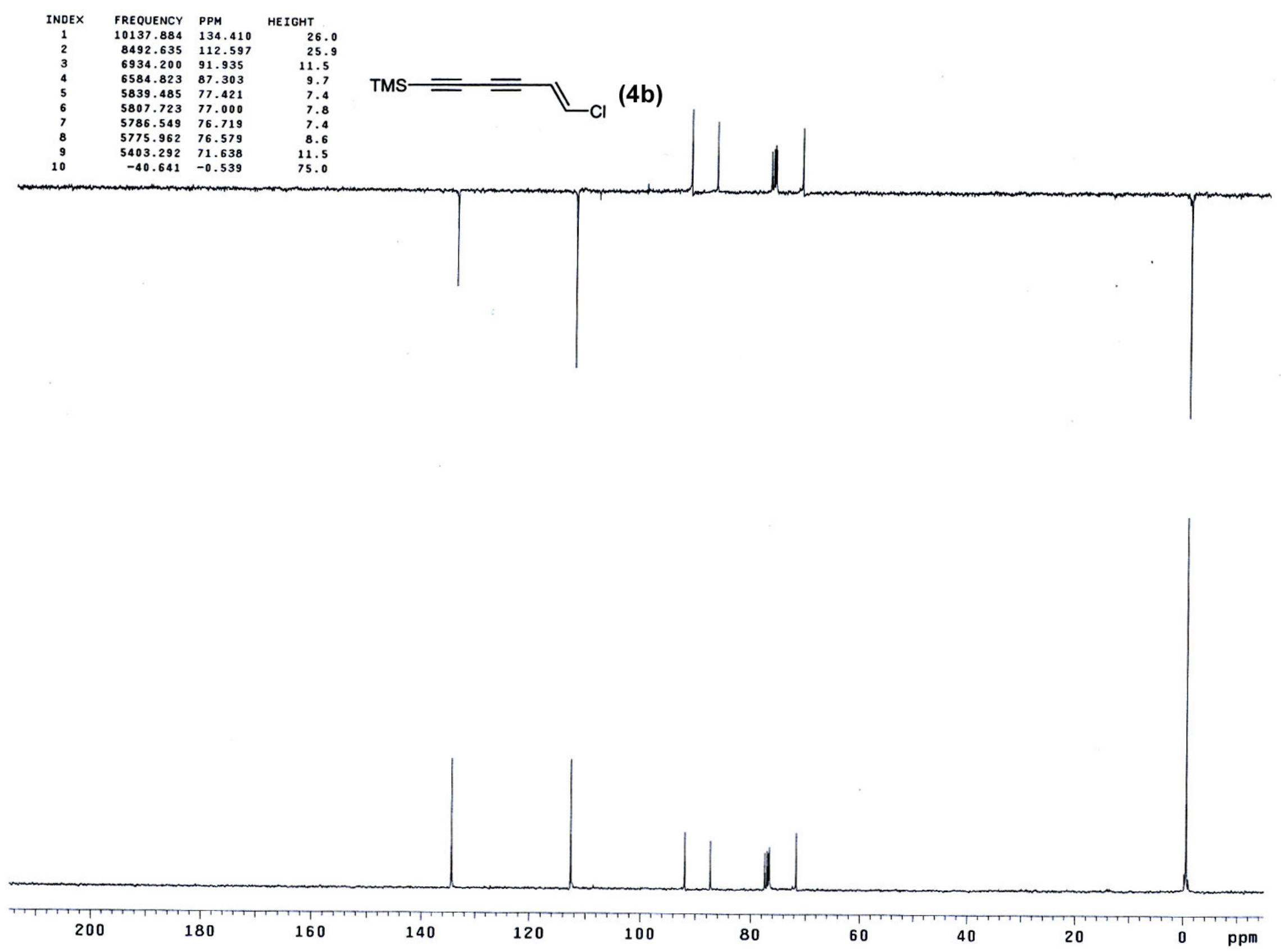


$\begin{array}{crlr}\text { INDEX } & \text { FREQUENCY } & \text { PPM } & \text { HEIGHT } \\ 1 & 2009.021 & 6.697 & 10.4 \\ 2 & 1995.545 & 6.652 & 11.9 \\ 3 & 1802.202 & 6.008 & 6.4 \\ 4 & 1788.726 & 5.963 & 5.9 \\ 5 & 1787.554 & 5.959 & 5.5 \\ 6 & 608.742 & 2.029 & 50.0\end{array}$

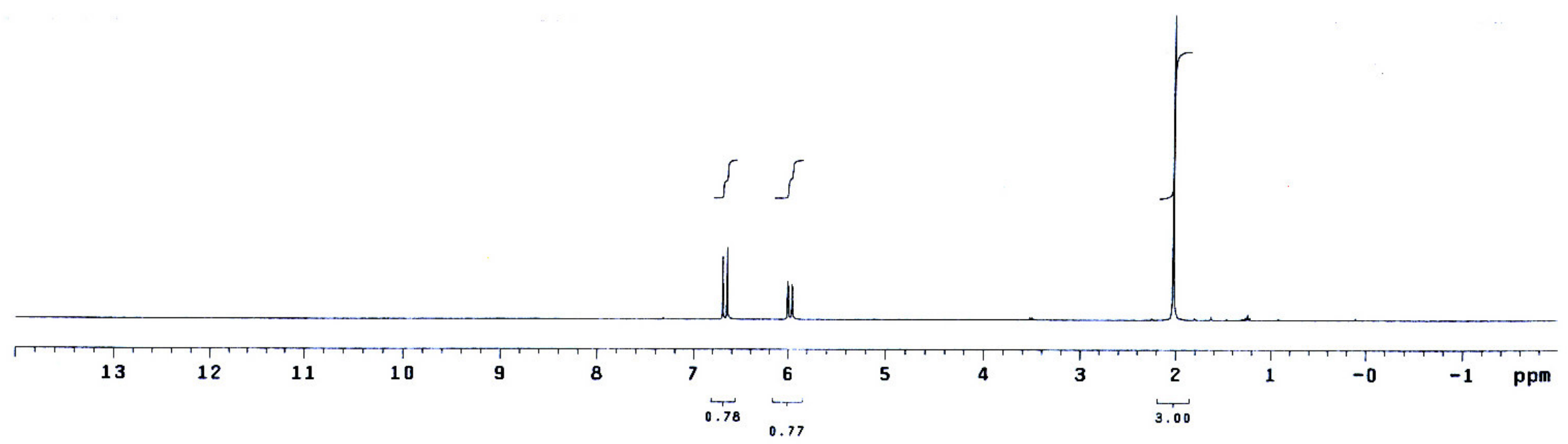



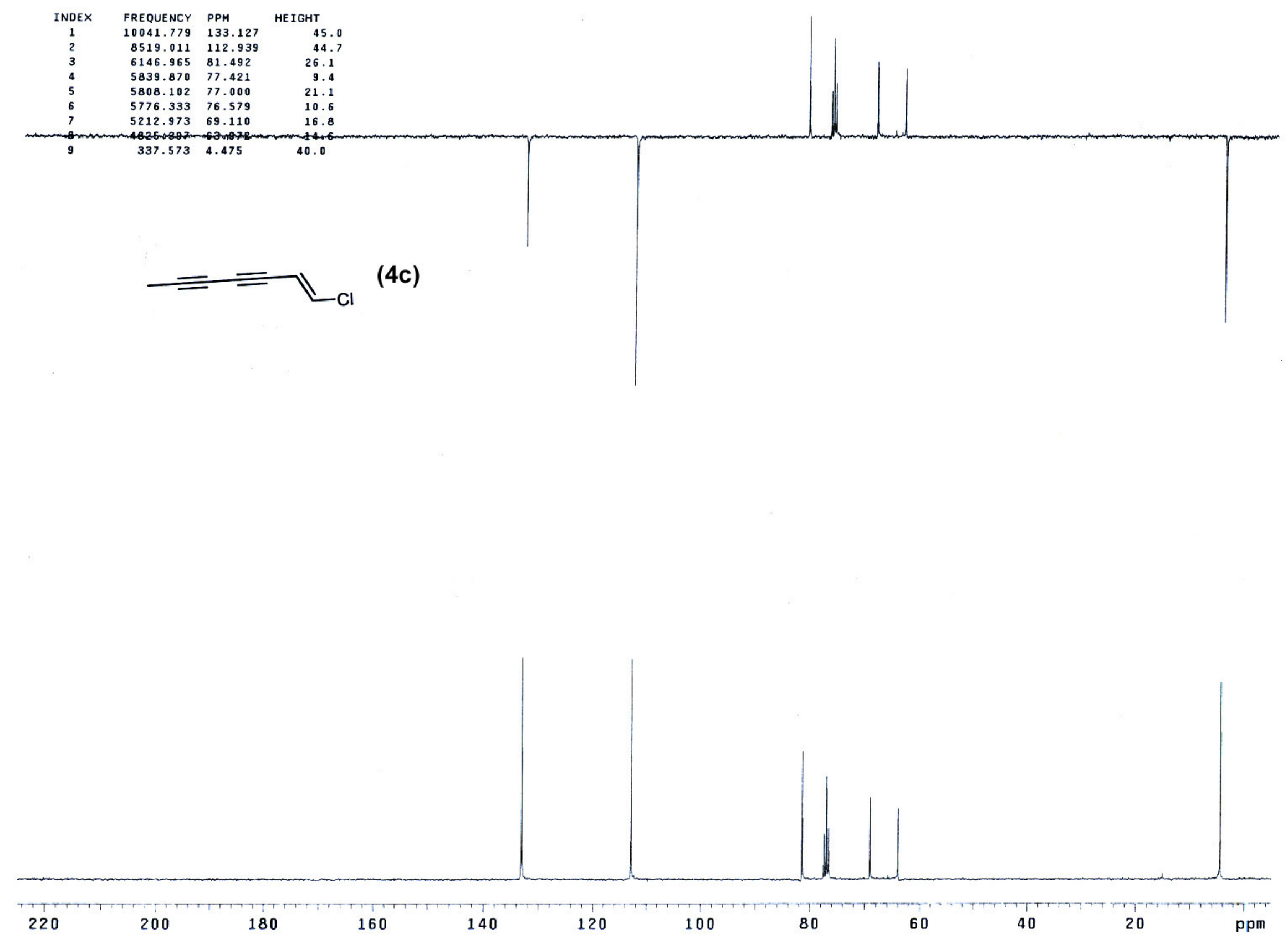


$\begin{array}{crlr}\text { INDEX } & \text { FREQUENCY } & \text { PPM } & \text { HEIGHT } \\ 1 & 1993.212 & 6.644 & 4.3 \\ 2 & 1989.111 & 6.631 & 5.5 \\ 3 & 1977.393 & 6.592 & 5.1 \\ 4 & 1973.292 & 6.578 & 6.6 \\ 5 & 1779.948 & 5.934 & 6.9 \\ 6 & 1765.301 & 5.885 & 5.7 \\ 7 & 1764.129 & 5.881 & 5.8 \\ 8 & 1289.558 & 4.299 & 7.6 \\ 9 & 1275.496 & 4.252 & 20.6 \\ 10 & 619.299 & 2.064 & 75.0\end{array}$
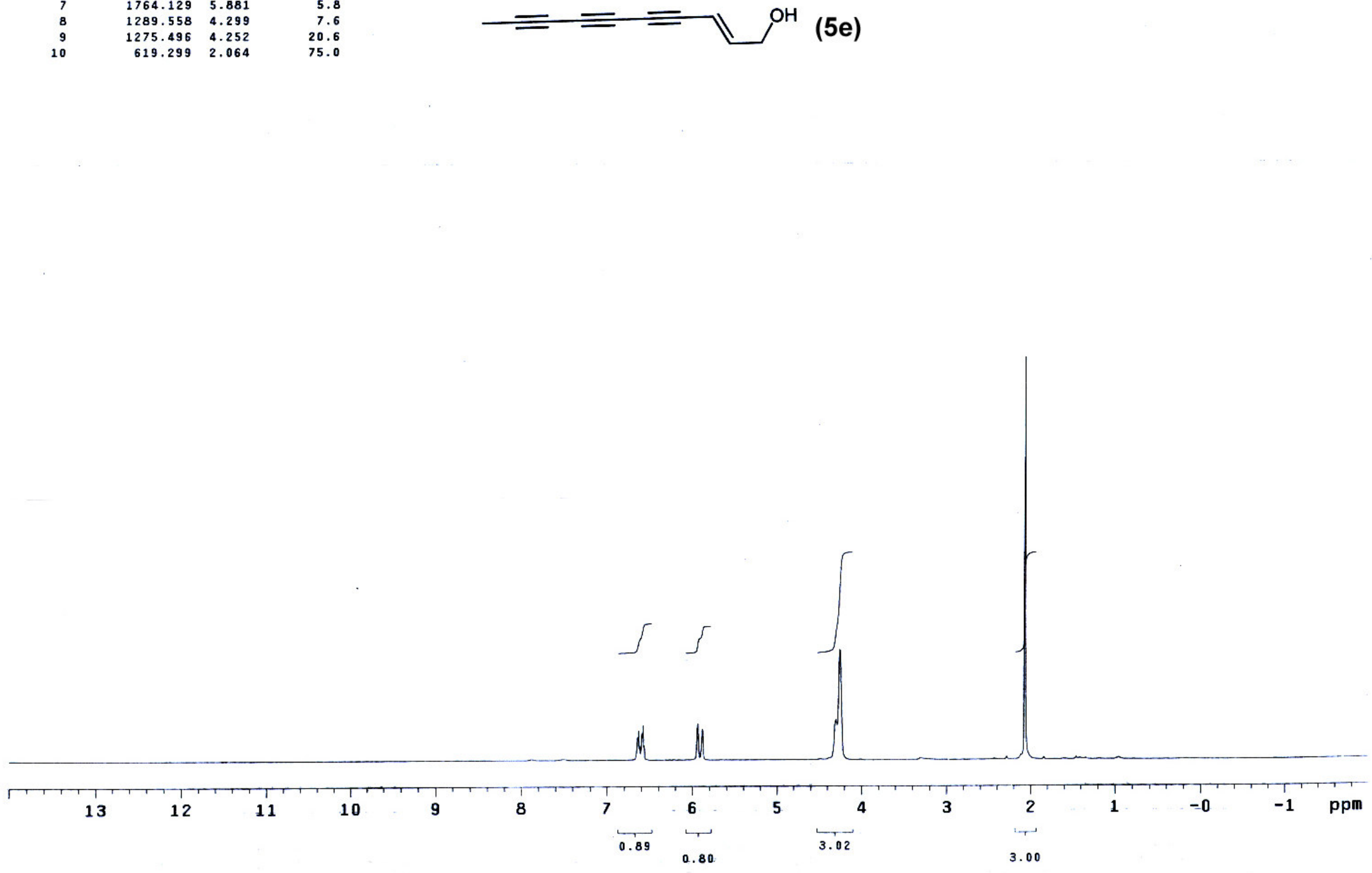

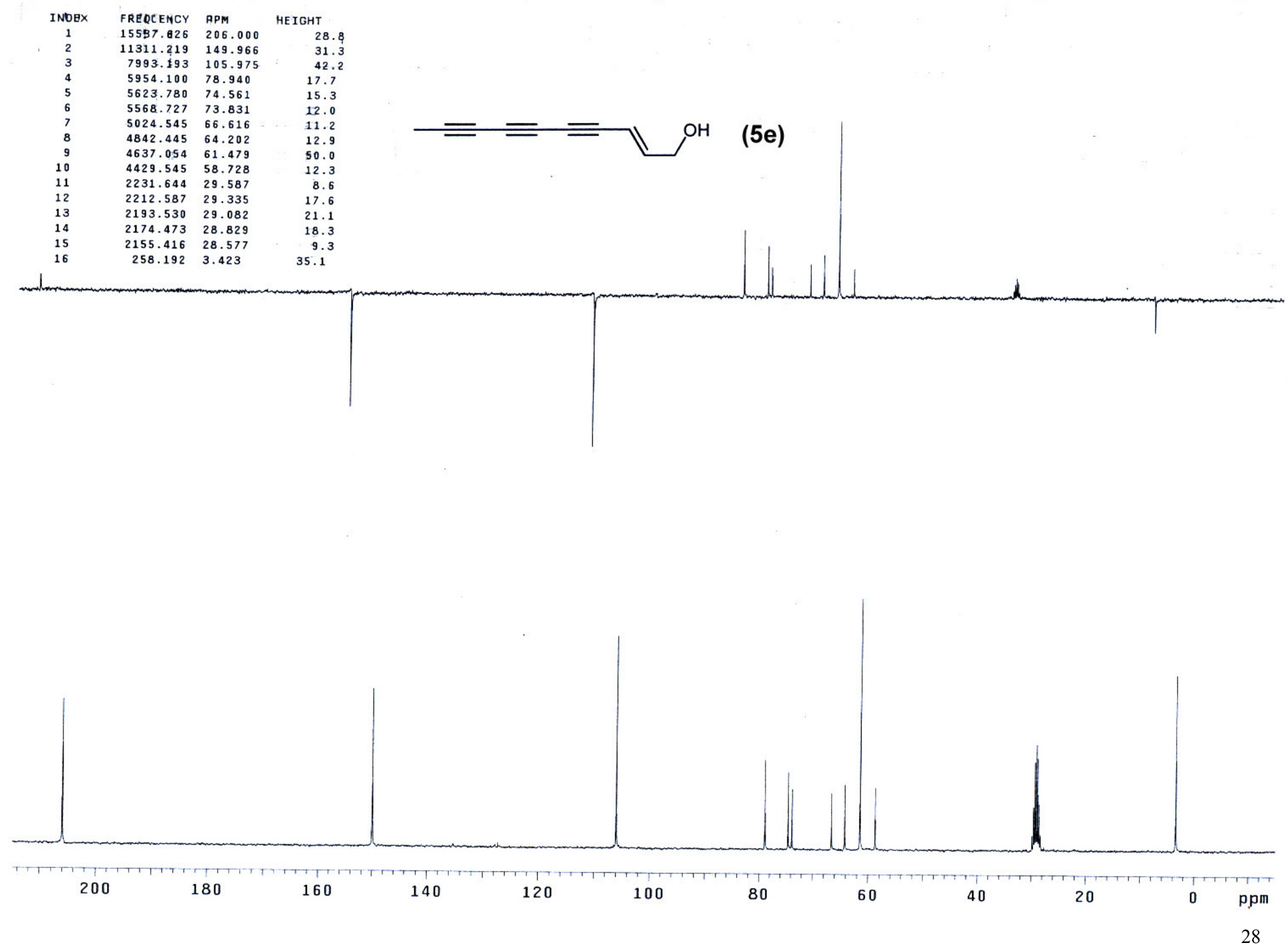


$\begin{array}{crlr}\text { INDEX } & \text { FREQUENCY } & \text { PPM } & \text { HEIGHT } \\ 1 & 1935.165 & 6.451 & 2.1 \\ 2 & 1931.064 & 6.438 & 4.6 \\ 3 & 1926.963 & 6.424 & 1.9 \\ 4 & 1919.347 & 6.399 & 2.5 \\ 5 & 1915.246 & 6.385 & 5.1 \\ 6 & 1911.730 & 6.373 & 2.3 \\ 7 & 1745.933 & 5.821 & 1.6 \\ 8 & 1743.590 & 5.813 & 3.0 \\ 9 & 1741.832 & 5.807 & 1.6 \\ 10 & 1730.115 & 5.768 & 1.3 \\ 11 & 1728.358 & 5.762 & 2.5 \\ 12 & 1726.014 & 5.754 & 1.4 \\ 13 & 1276.077 & 4.254 & 9.7 \\ 14 & 1273.734 & 4.246 & 10.8 \\ 15 & 1272.562 & 4.242 & 9.9 \\ 16 & 1270.219 & 4.235 & 8.6 \\ 17 & 1041.149 & 3.471 & 0.4 \\ 18 & 1034.119 & 3.448 & 0.5 \\ 19 & 590.040 & 1.967 & 42.4 \\ 20 & 363.314 & 1.211 & 0.5 \\ 21 & 356.284 & 1.188 & 1.0 \\ 22 & 349.254 & 1.164 & 0.5 \\ 23 & 329.335 & 1.098 & 0.8 \\ 24 & 287.739 & 0.959 & 1.2 \\ 25 & 270.163 & 0.901 & 9.6 \\ 26 & 267.234 & 0.891 & 150.0 \\ 27 & 260.790 & 0.869 & 1.1 \\ 28 & 204.548 & 0.682 & 0.8 \\ 29 & 75.074 & 0.250 & 0.5 \\ 30 & 36.407 & 0.121 & 1.0 \\ 31 & 19.417 & 0.065 & 5.8 \\ 32 & 16.488 & 0.055 & 137.8 \\ 33 & 13.559 & 0.045 & 4.1 \\ 34 & -43.269 & -0.144 & 0.5 \\ & & & \end{array}$

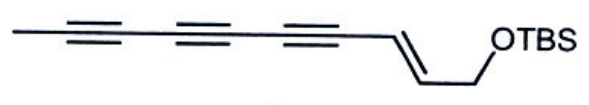

(5f)

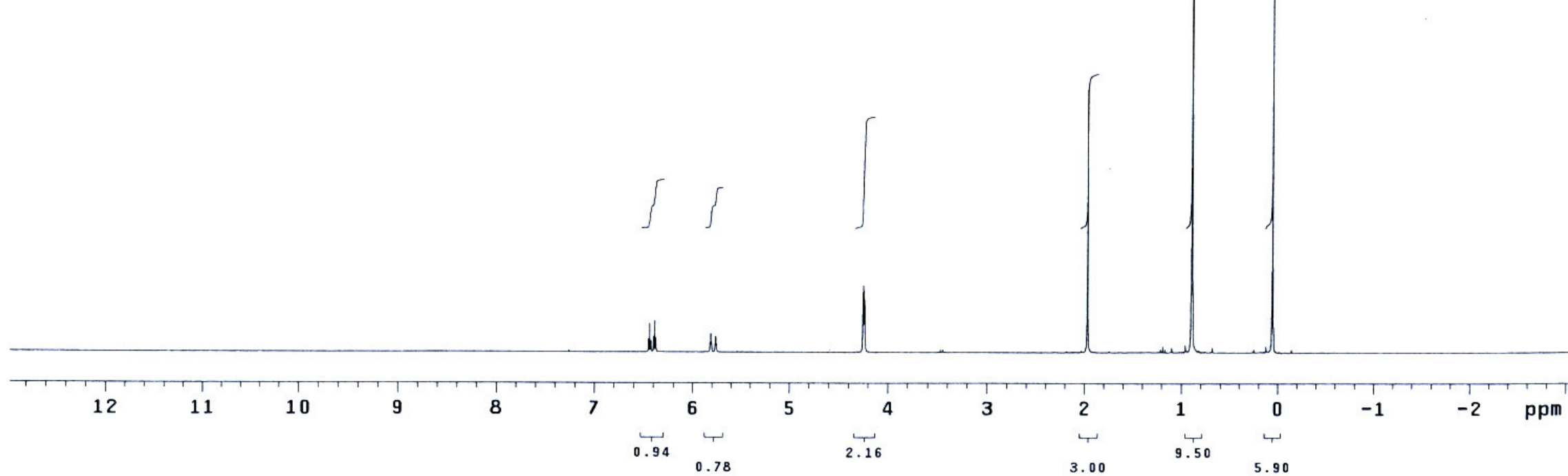



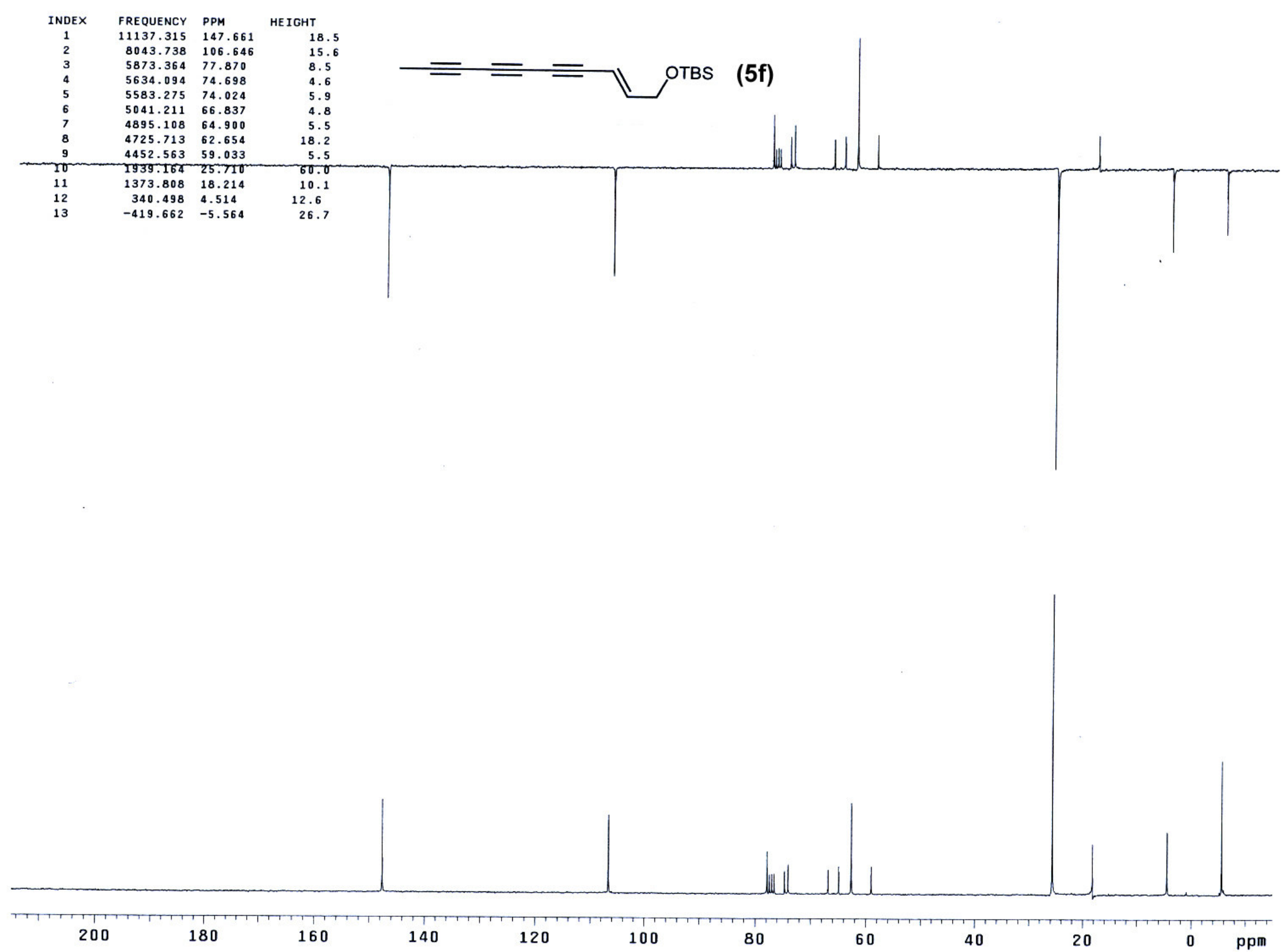
Highly Efficient and Selective Synthesis of Conjugated Triynes and Higher Oligoynes of ......

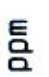

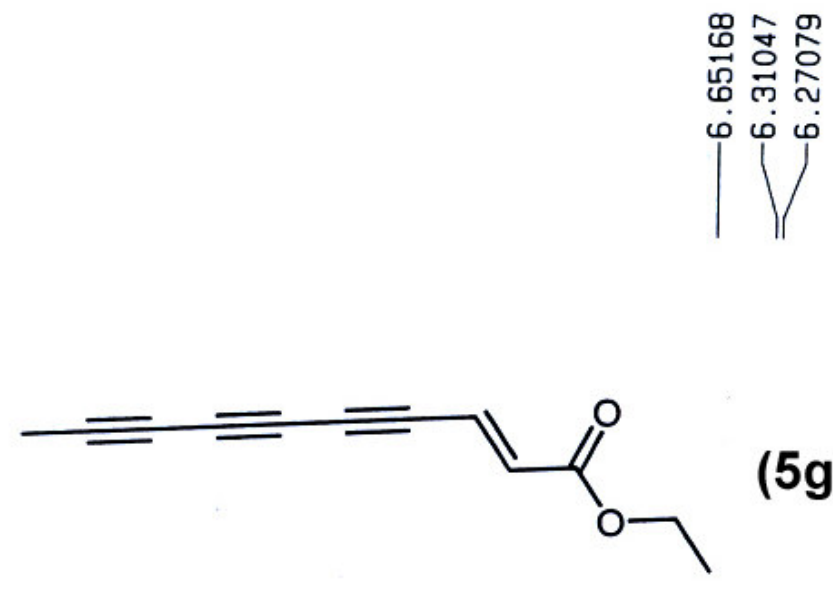

号 邑

$\nabla \nabla$

$\gamma$ (5g)
Métay, E., Hu, Q., Negishi, E.
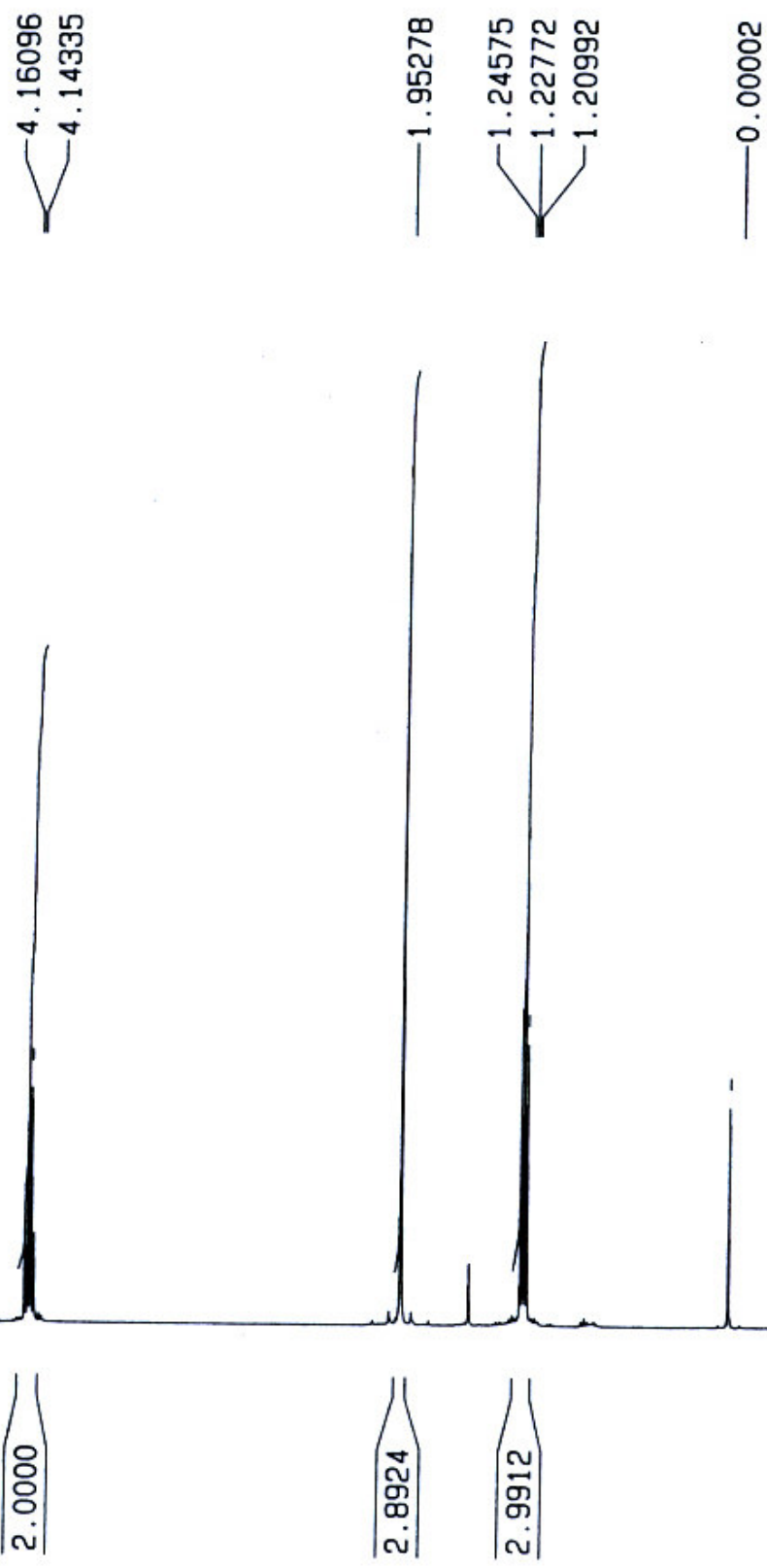

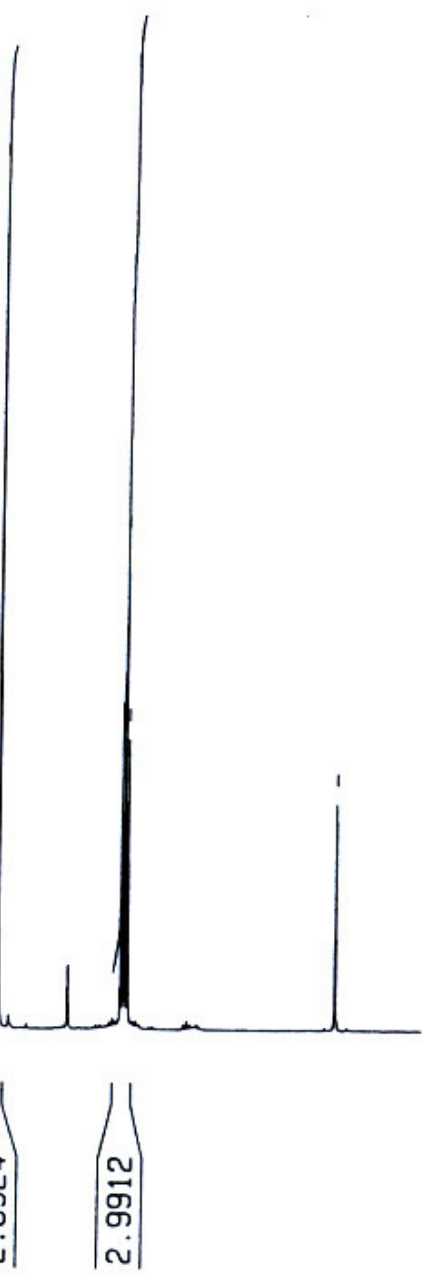

0 
Highly Efficient and Selective Synthesis of Conjugated Triynes and Higher Oligoynes of ......

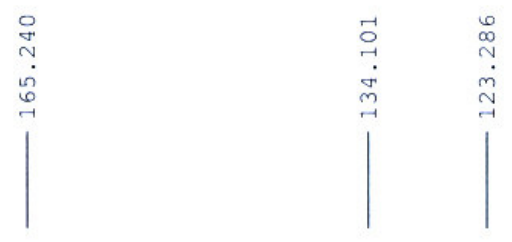

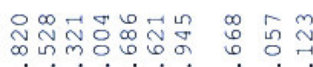

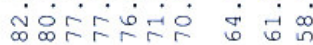

||$V||||$

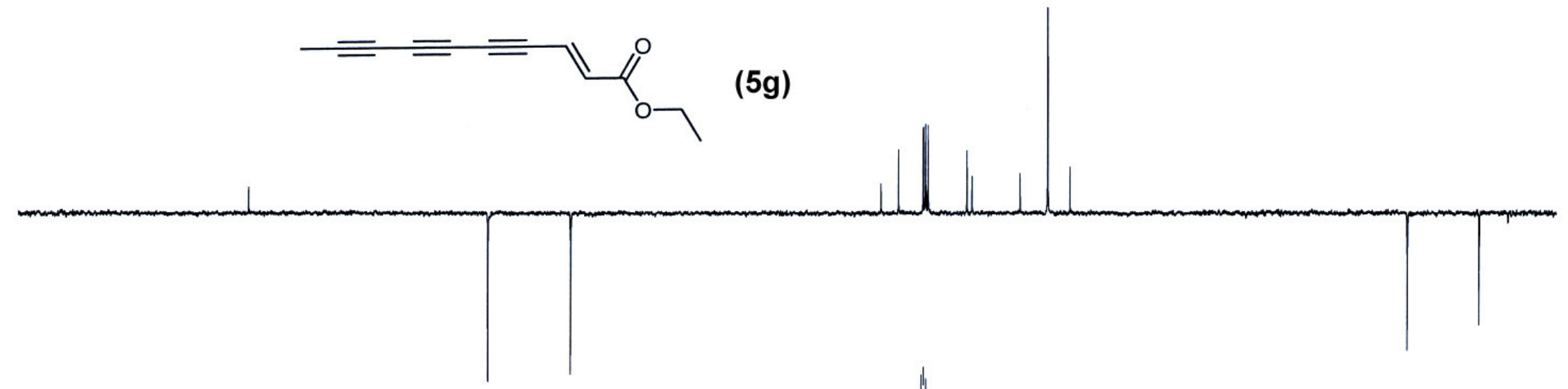

(5g)

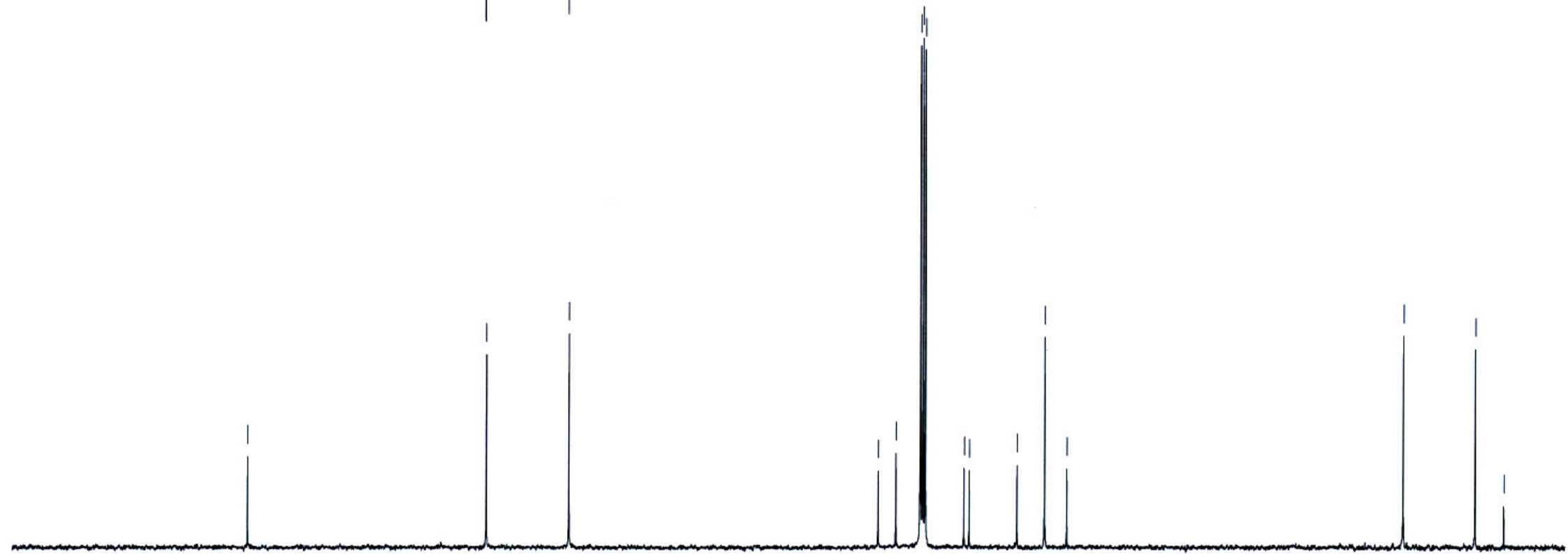




$\begin{array}{crlr}\text { INDEX } & \text { FREQUENCY } & \text { PPM } & \text { HEIGHT } \\ 1 & 2261.932 & 7.541 & 63.6 \\ 2 & 2254.901 & 7.517 & 70.2 \\ 3 & 2253.730 & 7.513 & 75.0 \\ 4 & 2218.578 & 7.396 & 28.0 \\ 5 & 2212.134 & 7.375 & 35.0 \\ 6 & 2205.690 & 7.353 & 69.7 \\ 7 & 2198.073 & 7.328 & 67.6 \\ 8 & 2191.629 & 7.306 & 23.4 \\ 9 & 2027.589 & 6.760 & 35.8 \\ 10 & 2014.115 & 6.715 & 43.6 \\ 11 & 1806.722 & 6.023 & 40.4 \\ 12 & 1793.247 & 5.978 & 38.5\end{array}$

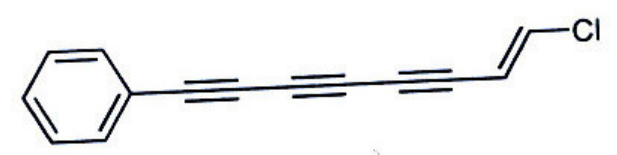

(6a)

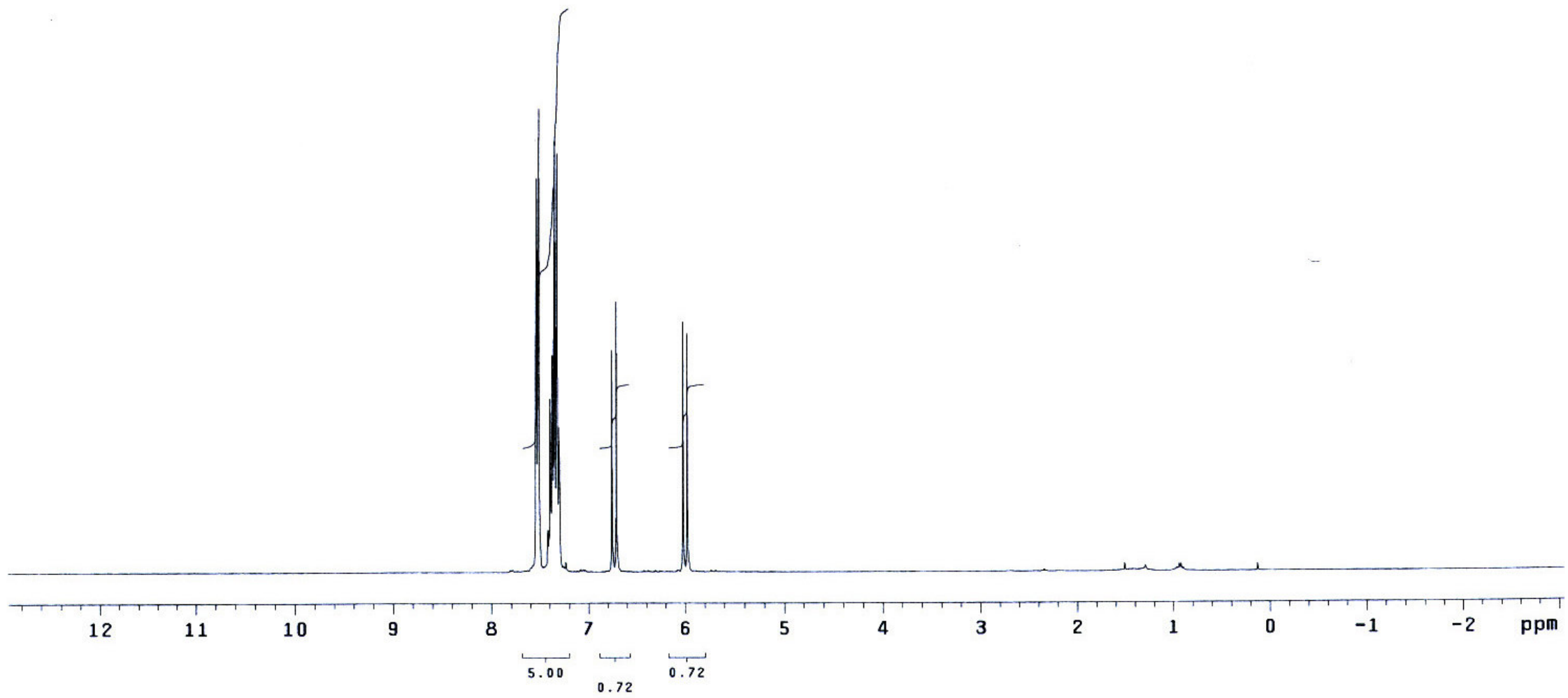




$\begin{array}{crlr}\text { INDEX } & \text { FREQUENCY } & \text { PPM } & \text { HEIGHT } \\ 1 & 10224.700 & 135.561 & 24.3 \\ 2 & 10023.544 & 132.894 & 65.0 \\ 3 & 9784.273 & 129.722 & 37.3 \\ 4 & 9684.754 & 128.402 & 53.4 \\ 5 & 9093.988 & 120.570 & 8.6 \\ 6 & 8471.461 & 112.316 & 25.0 \\ 7 & 5977.119 & 79.246 & 10.8 \\ 8 & 5837.368 & 77.393 & 4.8 \\ 9 & 5807.724 & 77.000 & 12.2 \\ 10 & 5773.845 & 76.551 & 5.7 \\ 11 & 5598.098 & 74.221 & 6.7 \\ 12 & 5547.279 & 73.547 & 11.0 \\ 13 & 5094.147 & 67.539 & 9.7 \\ 14 & 4973.453 & 65.939 & 9.5\end{array}$
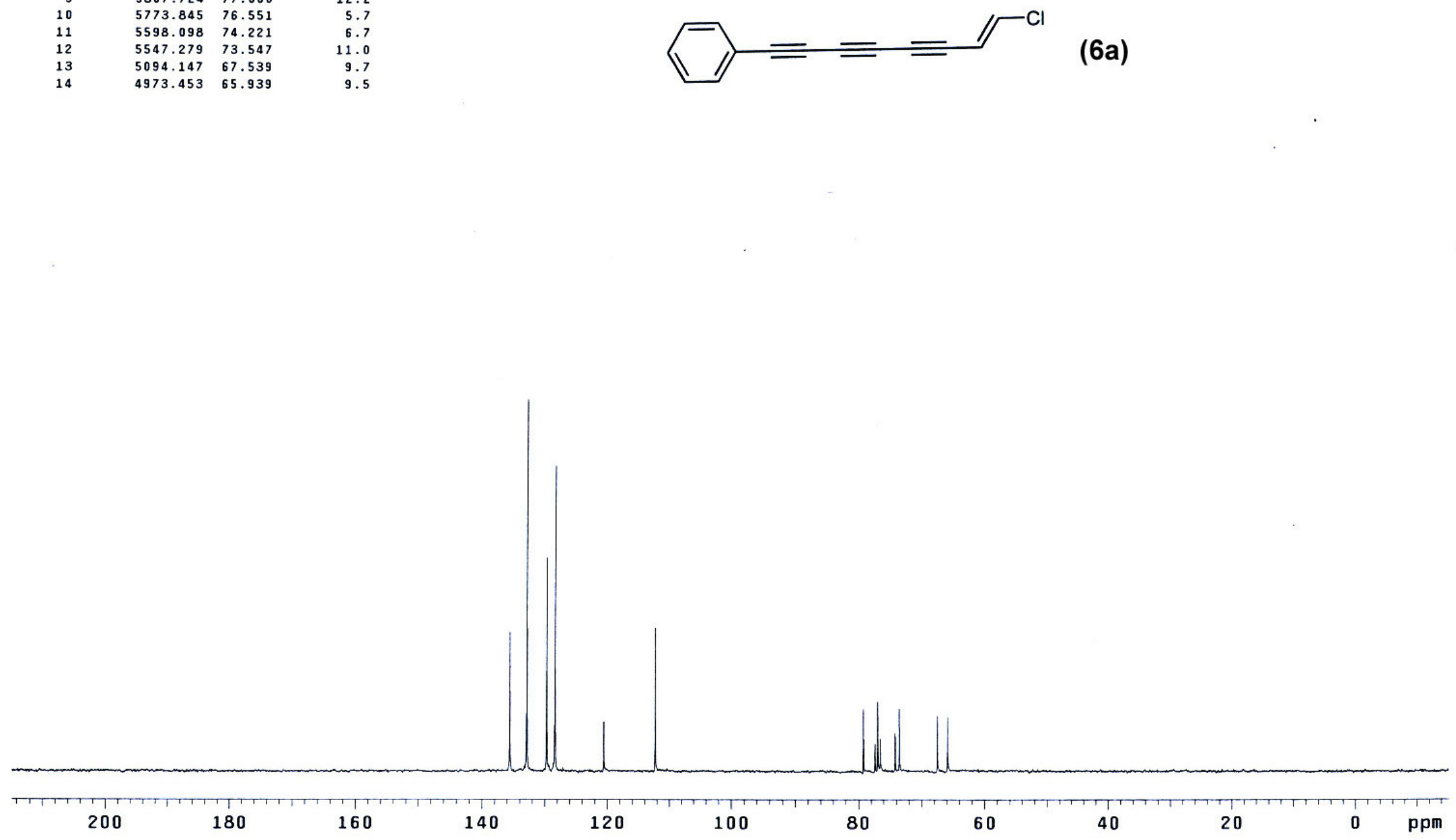


$\begin{array}{crlr}\text { INDEX } & \text { FREQUENCY } & \text { PPM } & \text { HEIGHT } \\ 1 & 2026.418 & 6.756 & 4.5 \\ 2 & 2012.943 & 6.711 & 4.3 \\ 3 & 1791.490 & 5.972 & 3.9 \\ 4 & 1778.015 & 5.928 & 4.0 \\ 5 & 60.873 & 0.203 & 4.3 \\ 6 & 57.357 & 0.191 & 100.0 \\ 7 & 53.842 & 0.179 & 3.7\end{array}$
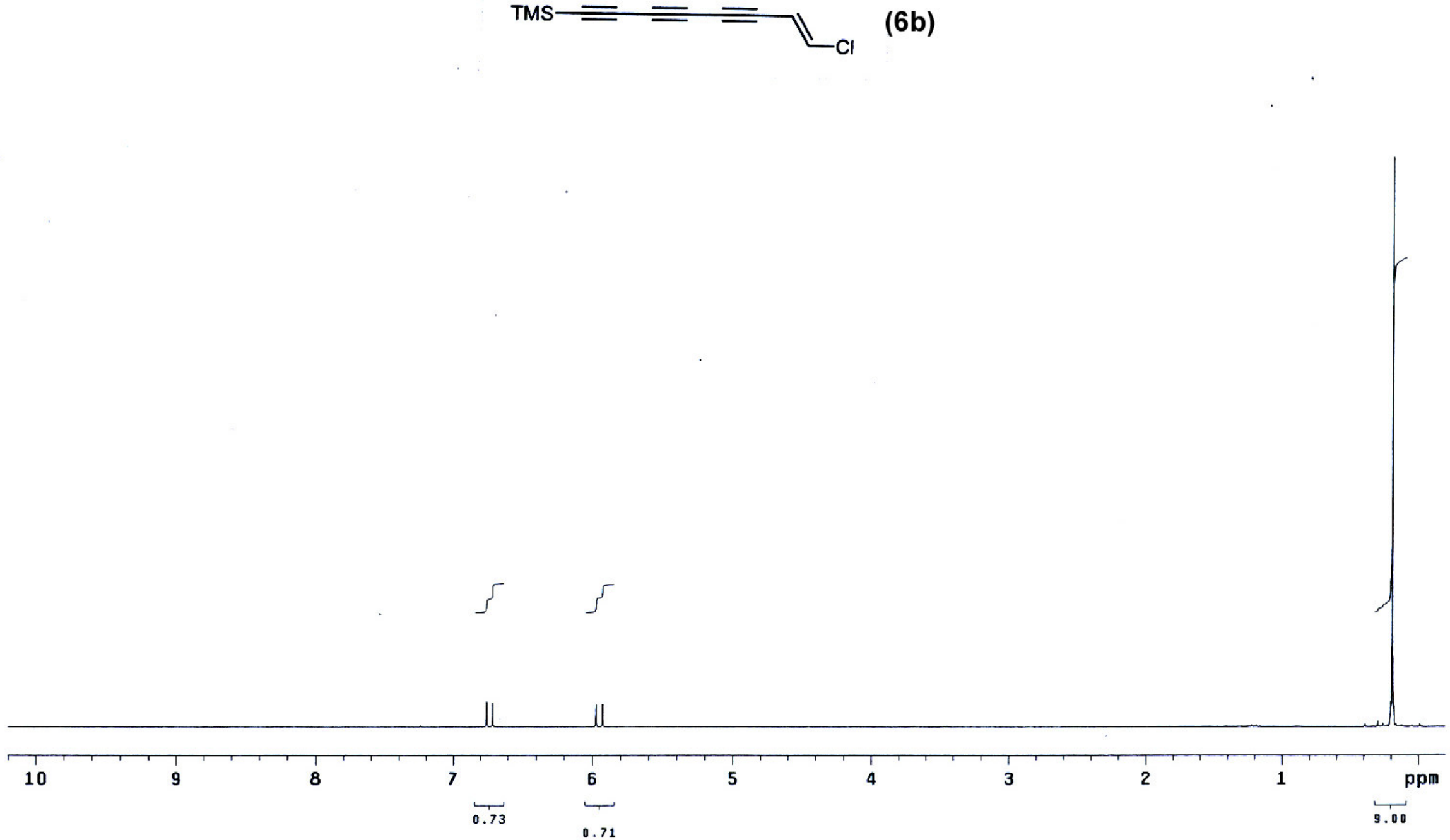

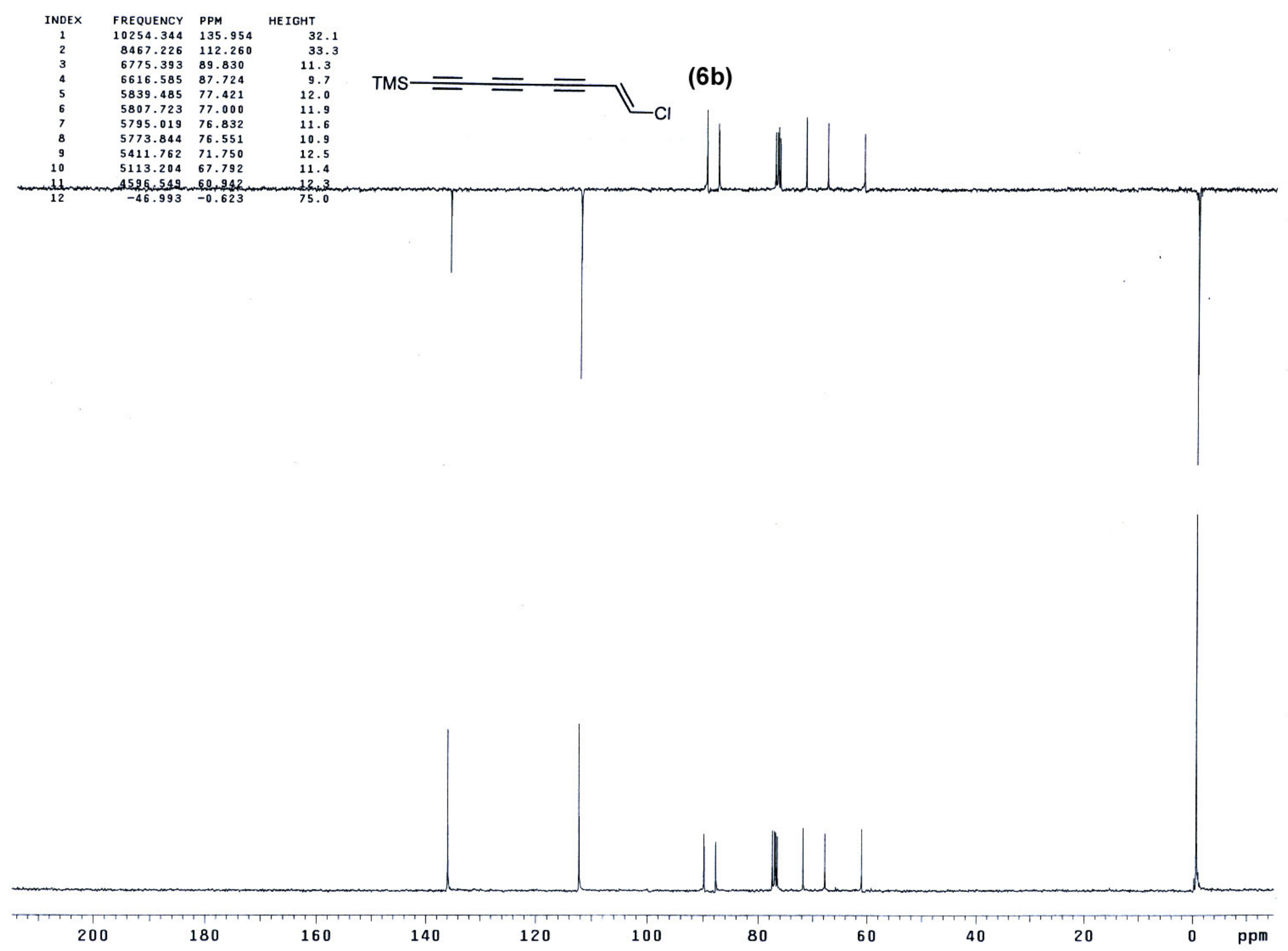


$\begin{array}{crlr}\text { INDEX } & \text { FREQUENCY } & \text { PPM } & \text { HEIGHT } \\ 1 & 2016.636 & 6.723 & 11.4 \\ 2 & 2002.574 & 6.676 & 13.1 \\ 3 & 1787.553 & 5.959 & 9.8 \\ 4 & 1774.077 & 5.914 & 9.1 \\ 5 & 592.336 & 1.975 & 75.0\end{array}$

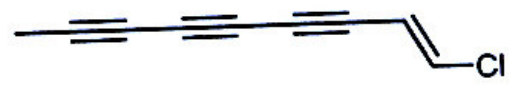

(6c)

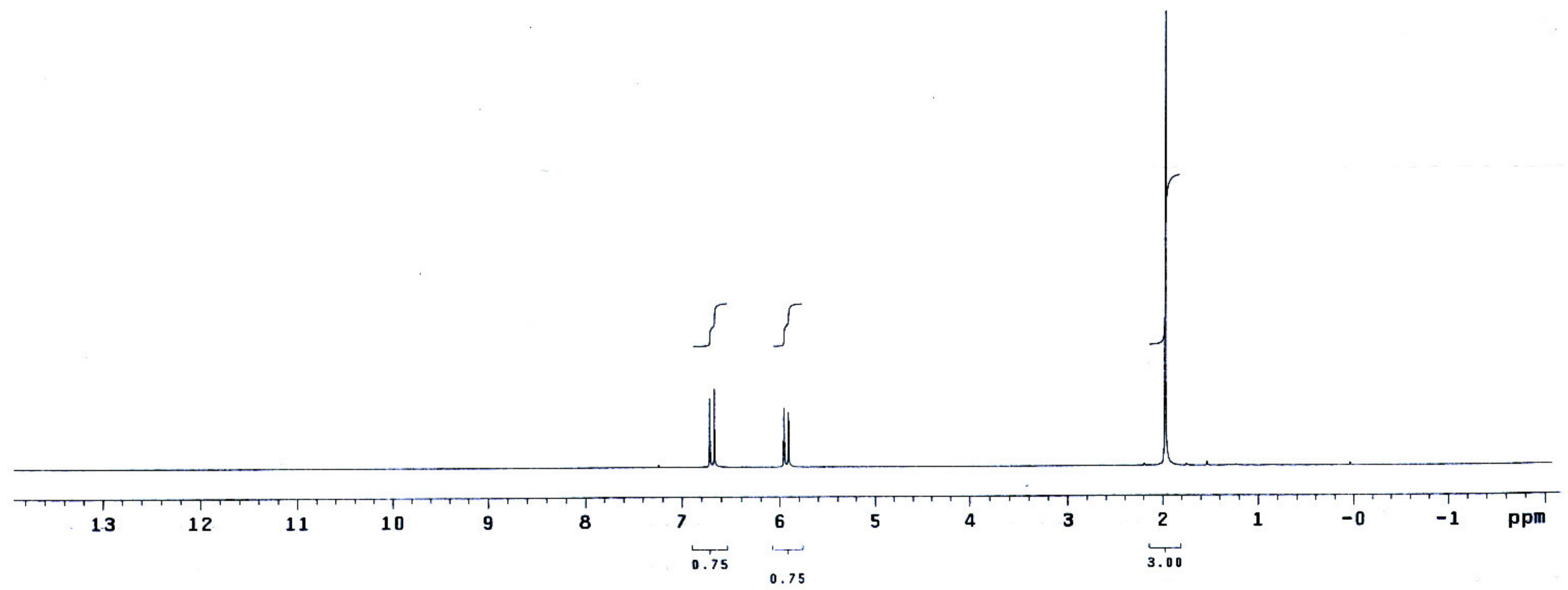



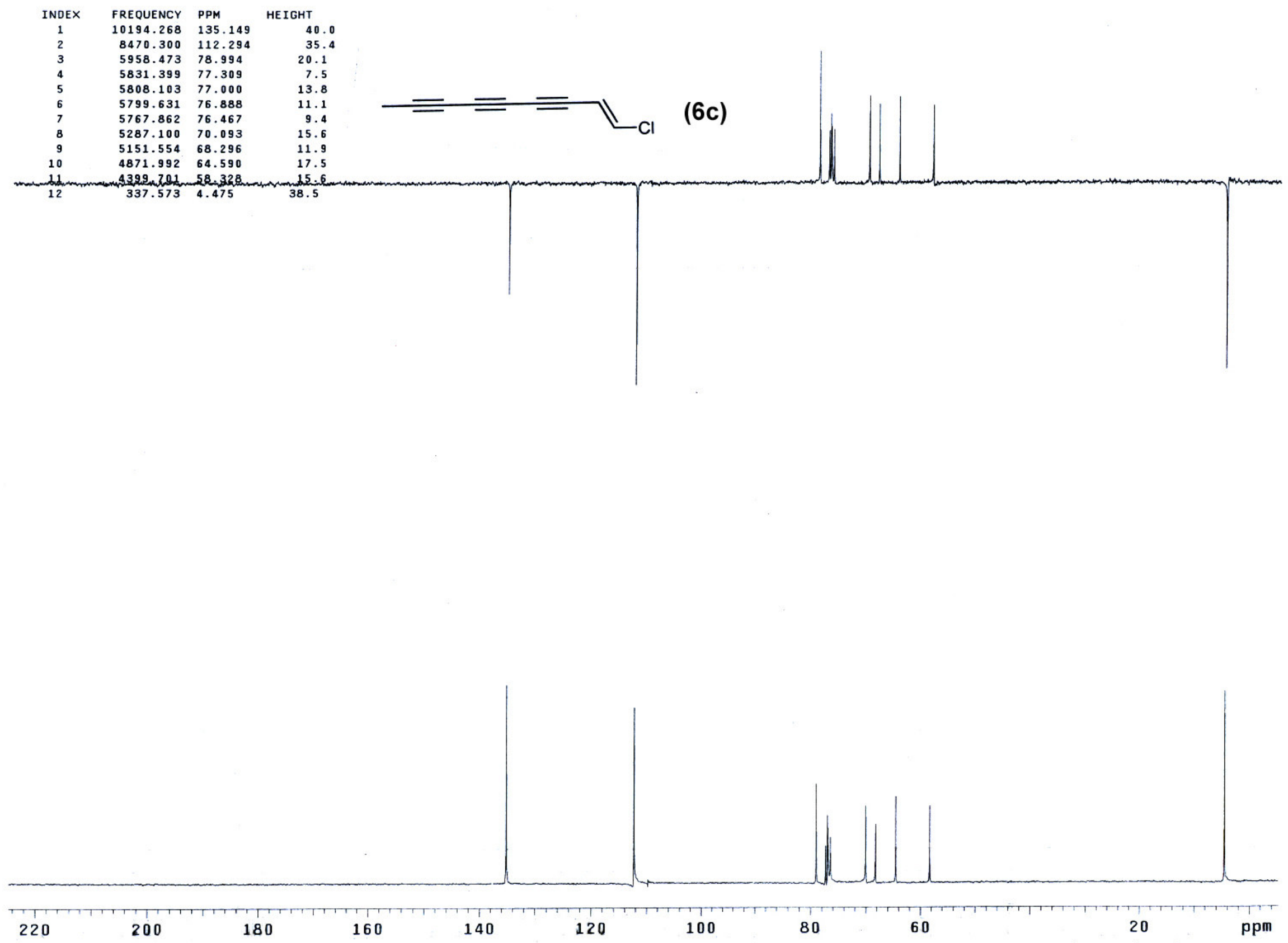


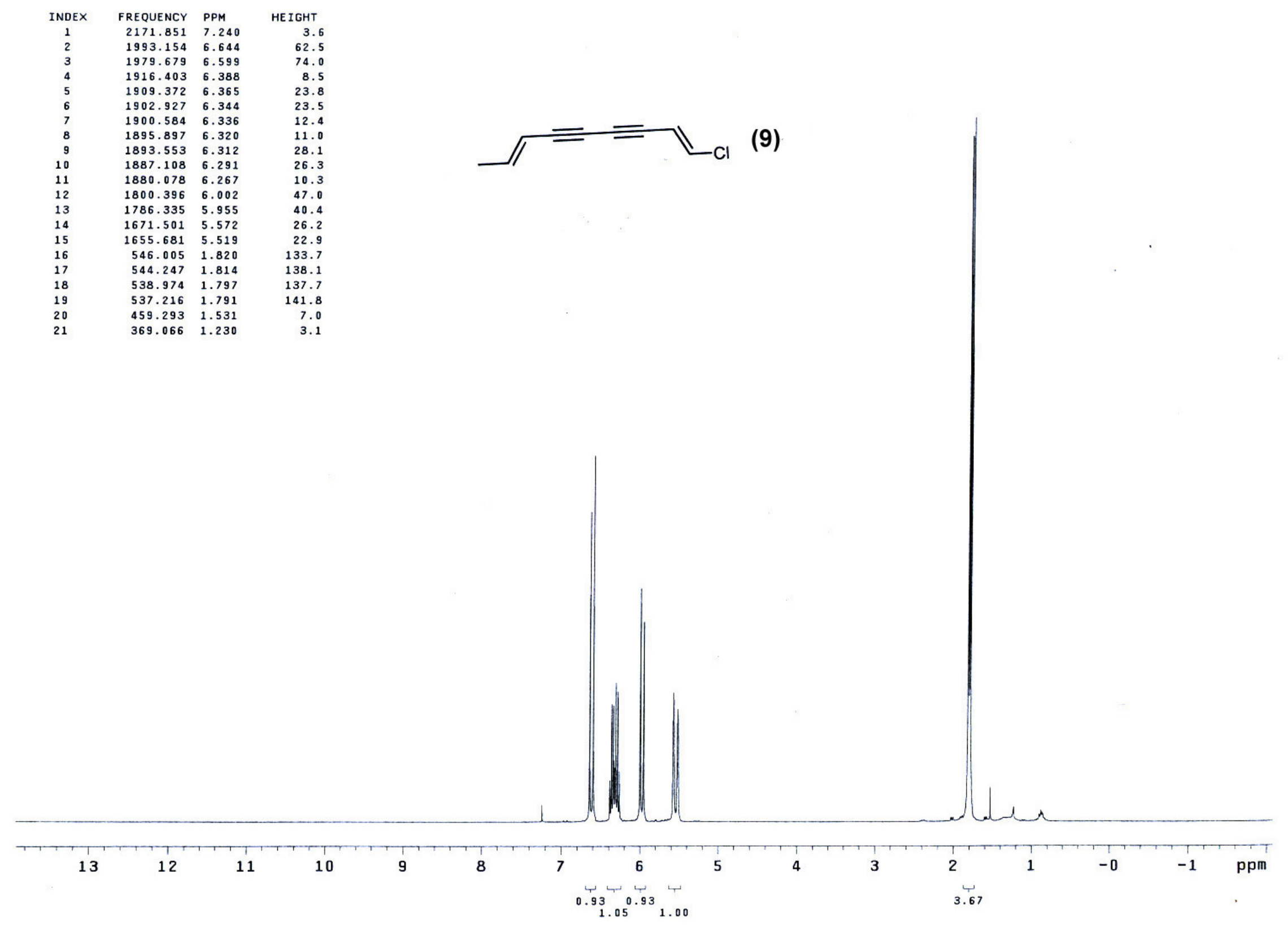



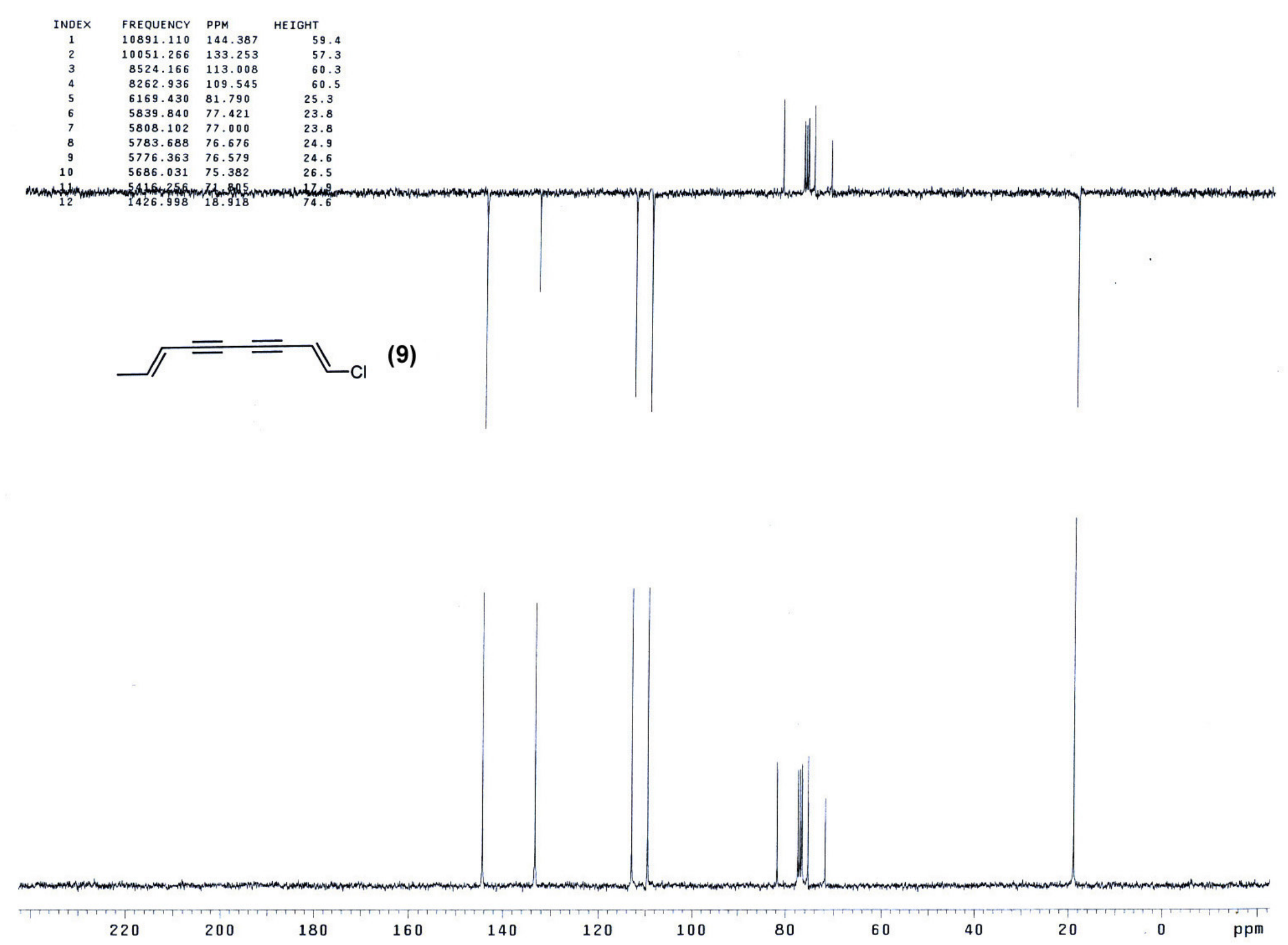


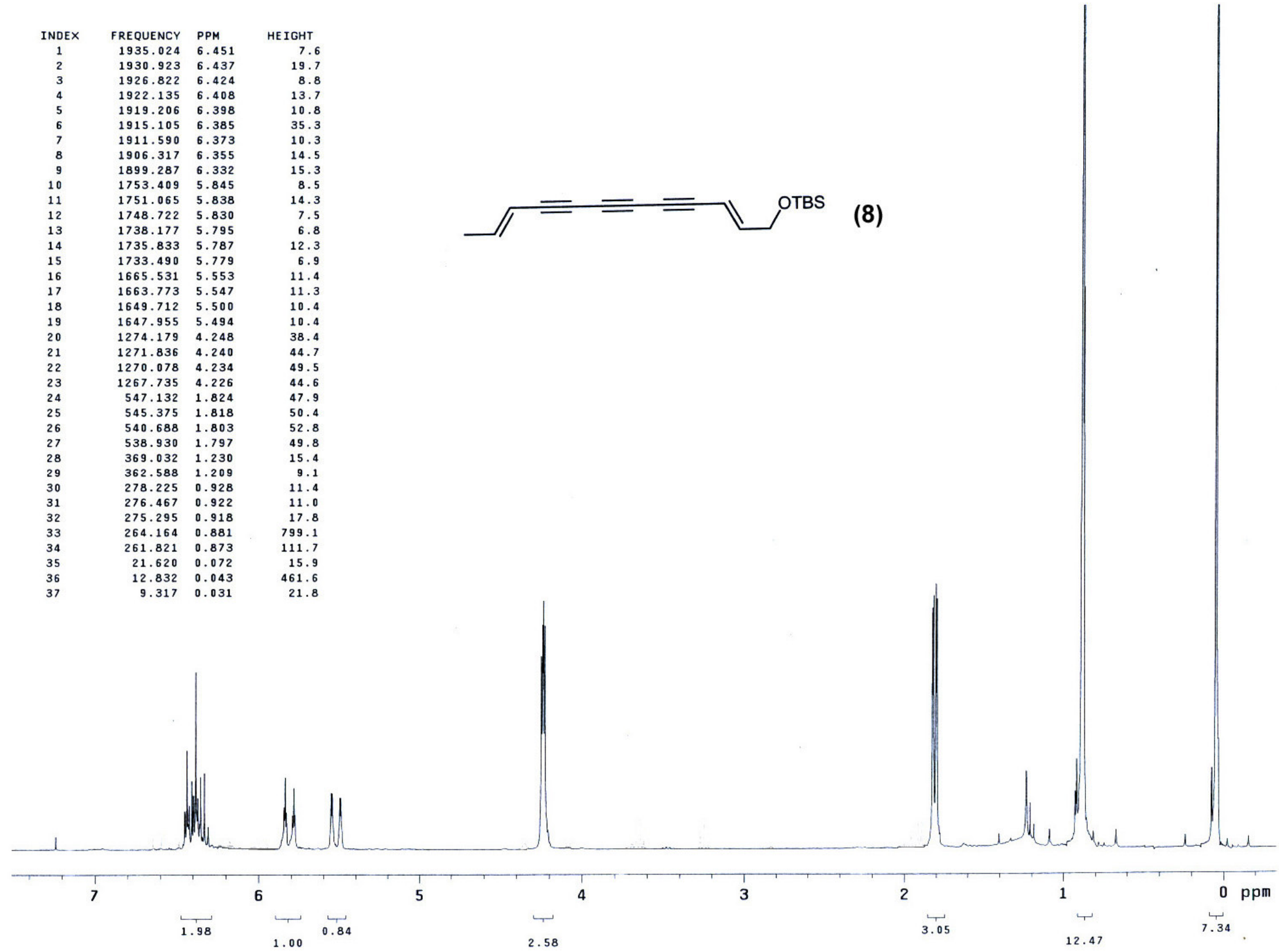



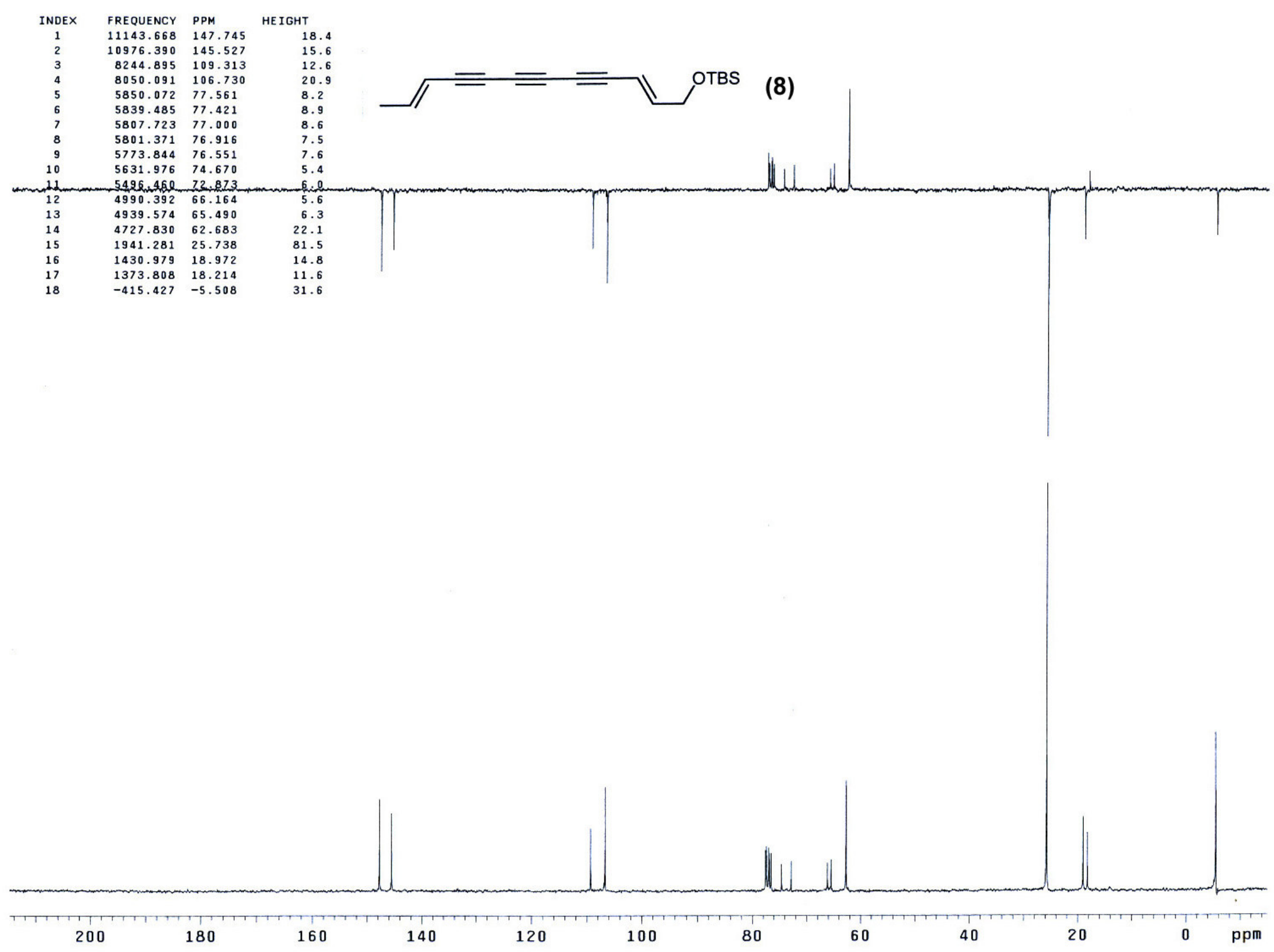


$\begin{array}{crlr}\text { INDEX } & \text { FREQUENCY } & \text { PPM } & \text { HEIGHT } \\ 1 & 2269.688 & 7.567 & 39.8 \\ 2 & 2262.658 & 7.543 & 64.0 \\ 3 & 2257.971 & 7.528 & 42.8 \\ 4 & 2242.153 & 7.475 & 11.7 \\ 5 & 2239.810 & 7.467 & 15.3 \\ 6 & 2237.466 & 7.459 & 13.5 \\ 7 & 2233.951 & 7.447 & 48.1 \\ 8 & 2226.921 & 7.424 & 75.0 \\ 9 & 2220.476 & 7.403 & 30.4 \\ 10 & 2212.274 & 7.375 & 42.7 \\ 11 & 2204.658 & 7.350 & 47.5 \\ 12 & 2197.628 & 7.326 & 16.8 \\ 13 & 1656.298 & 5.522 & 30.8 \\ 14 & 989.594 & 3.299 & 12.2\end{array}$
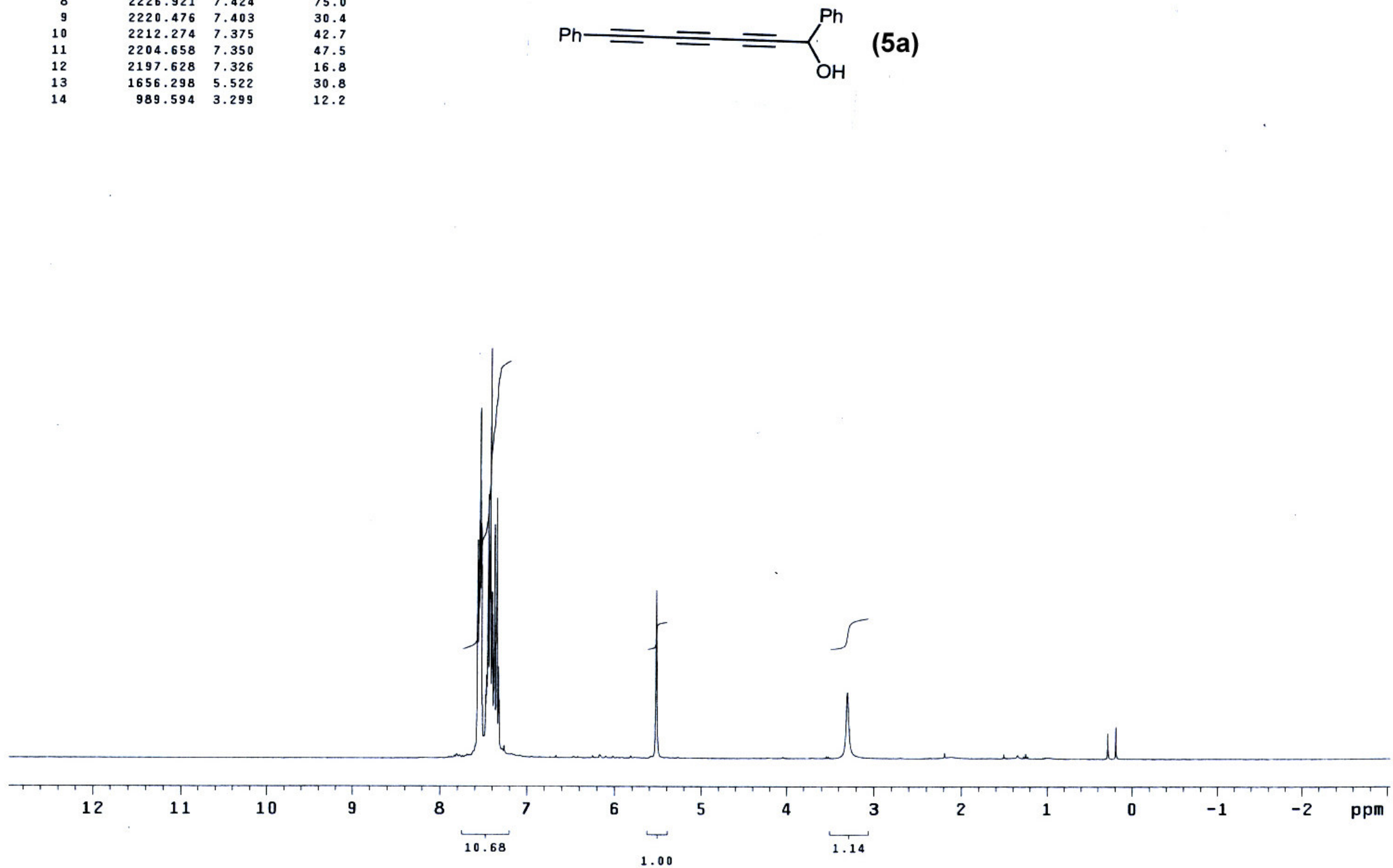


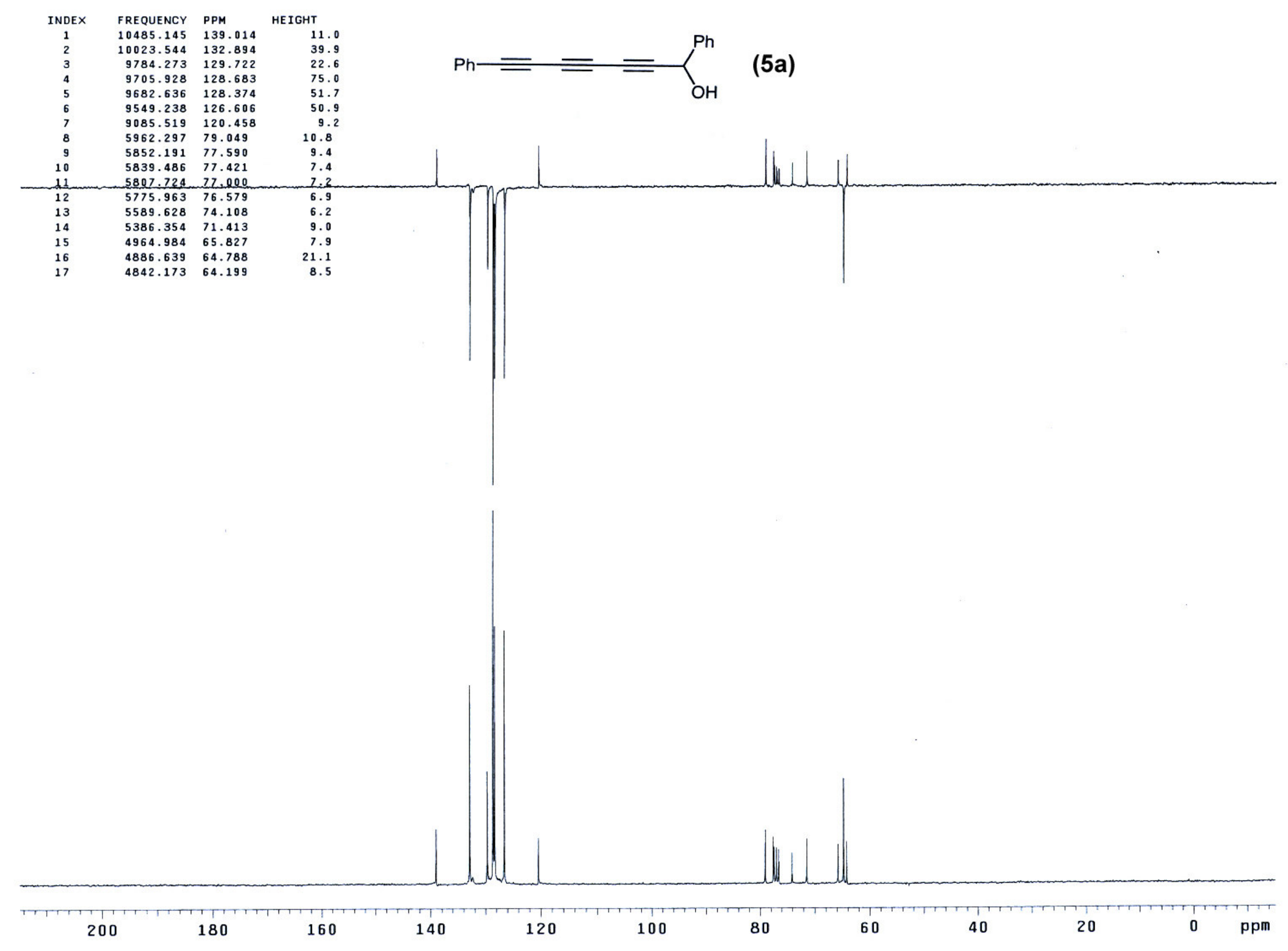




$\begin{array}{crlr}\text { INDEX } & \text { FREQUENCY } & \text { PPM } & \text { HEIGHT } \\ 1 & 2258.608 & 7.529 & 5.3 \\ 2 & 2257.437 & 7.525 & 5.2 \\ 3 & 2250.992 & 7.504 & 7.2 \\ 4 & 2204.706 & 7.350 & 5.6 \\ 5 & 2198.848 & 7.330 & 5.6 \\ 6 & 2197.676 & 7.326 & 5.4 \\ 7 & 296.461 & 0.988 & 61.2 \\ 8 & 295.289 & 0.984 & 75.0 \\ 9 & 54.488 & 0.182 & 42.7 \\ 10 & 53.316 & 0.178 & 52.6\end{array}$
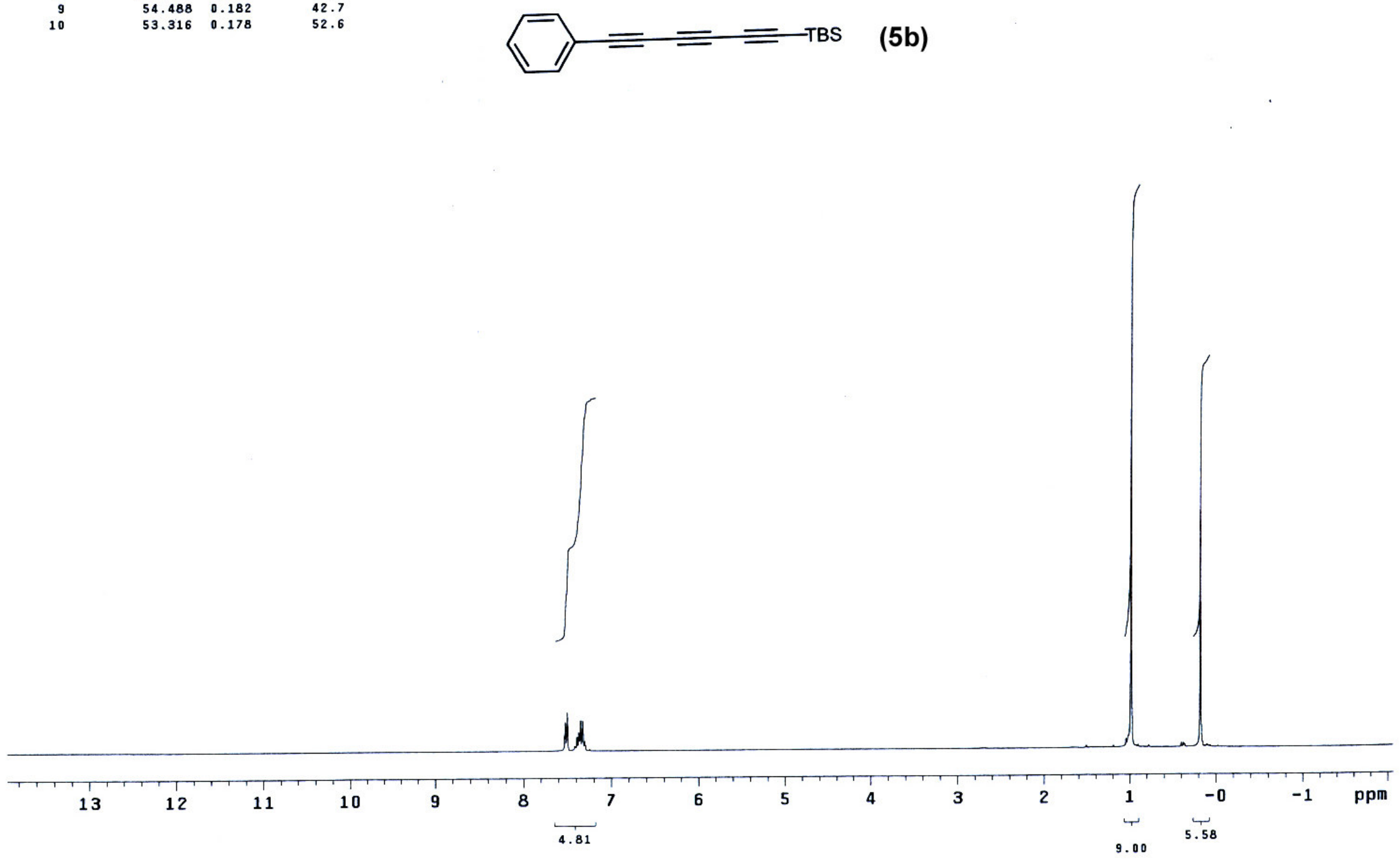


$\begin{array}{crlr}\text { INDEX } & \text { FREQUENCY } & \text { PPM } & \text { HEIGHT } \\ 1 & 10033.456 & 133.017 & 63.6 \\ 2 & 9785.315 & 129.727 & 28.9 \\ 3 & 9689.074 & 128.451 & 43.5 \\ 4 & 9108.145 & 120.750 & 20.2 \\ 5 & 6689.350 & 88.683 & 18.7 \\ 6 & 6626.735 & 87.853 & 19.6 \\ 7 & 5839.409 & 77.415 & 19.8 \\ 8 & 5808.102 & 77.000 & 20.1 \\ 9 & 5788.390 & 76.739 & 22.5 \\ 10 & 5775.635 & 76.570 & 20.7 \\ 11 & 5604.023 & 74.294 & 16.4 \\ 12 & 5054.402 & 67.008 & 17.0 \\ 13 & 4614.938 & 61.182 & 17.2 \\ 14 & 1963.075 & 26.025 & 56.4 \\ 15 & 1957.278 & 25.948 & 59.9 \\ 16 & 1266.193 & 16.786 & 30.4 \\ 17 & -374.552 & -4.966 & 70.0\end{array}$
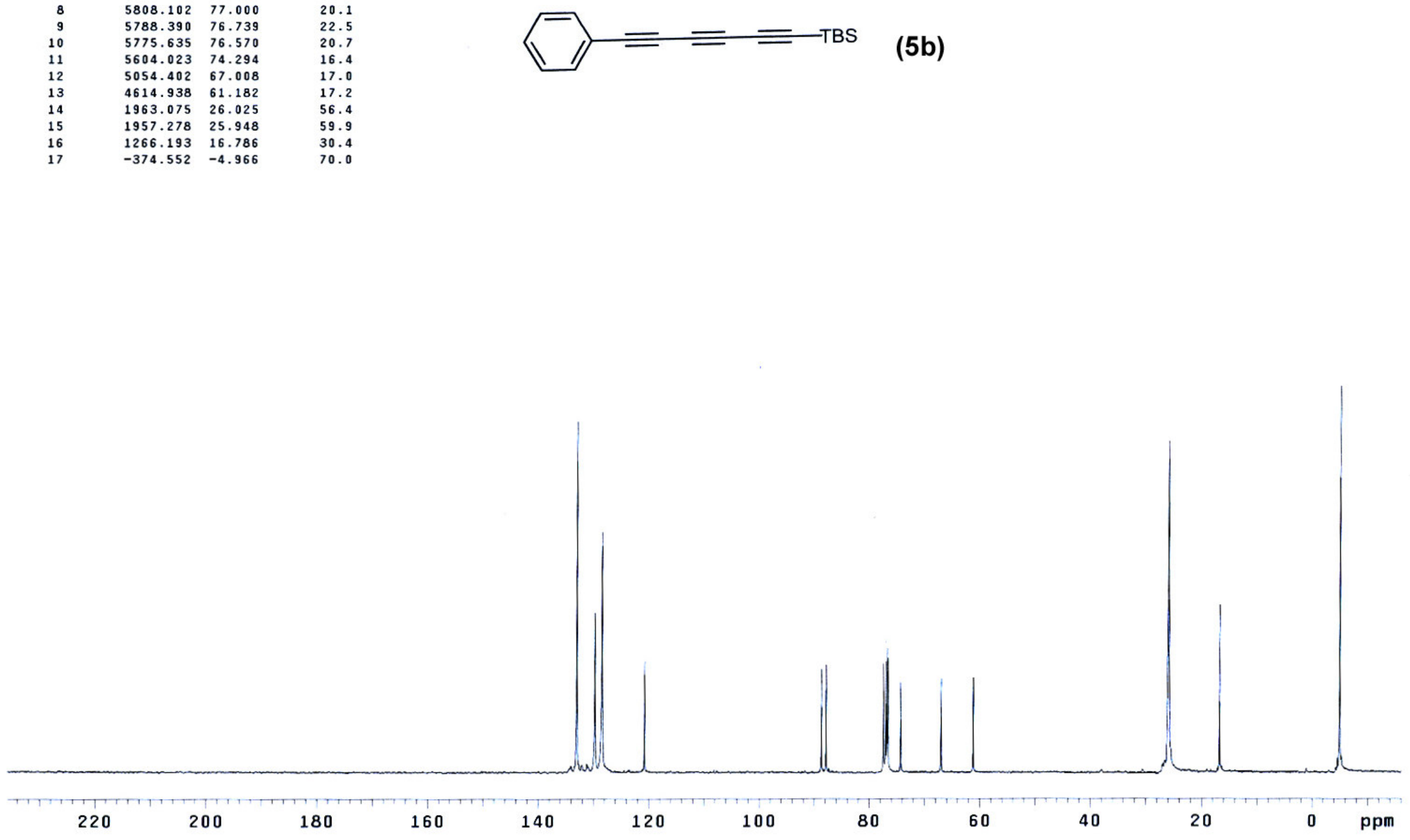


$\begin{array}{crlr}\text { INDEX } & \text { FREQUENCY } & \text { PPM } & \text { HEIGHT } \\ 1 & 2257.437 & 7.525 & 9.0 \\ 2 & 2256.265 & 7.521 & 13.0 \\ 3 & 2254.507 & 7.516 & 7.6 \\ 4 & 2249.820 & 7.500 & 15.7 \\ 5 & 2248.063 & 7.494 & 15.9 \\ 6 & 2214.667 & 7.383 & 6.6 \\ 7 & 2209.394 & 7.365 & 5.6 \\ 8 & 2208.222 & 7.361 & 8.9 \\ 9 & 2205.879 & 7.353 & 6.8 \\ 10 & 2203.535 & 7.346 & 15.4 \\ 11 & 2196.504 & 7.322 & 15.2 \\ 12 & 2189.474 & 7.299 & 5.0 \\ 13 & 598.195 & 1.994 & 75.0\end{array}$
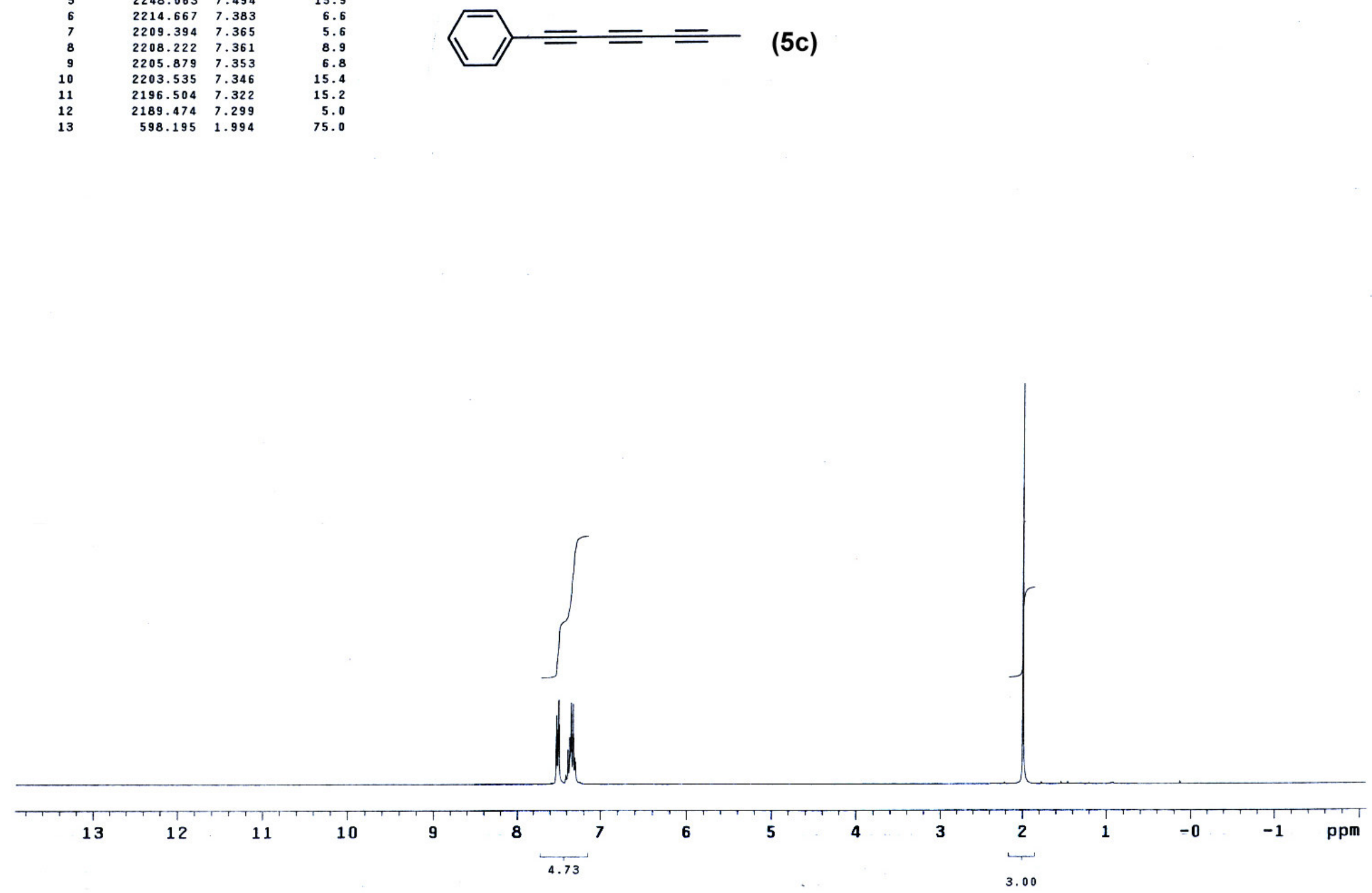

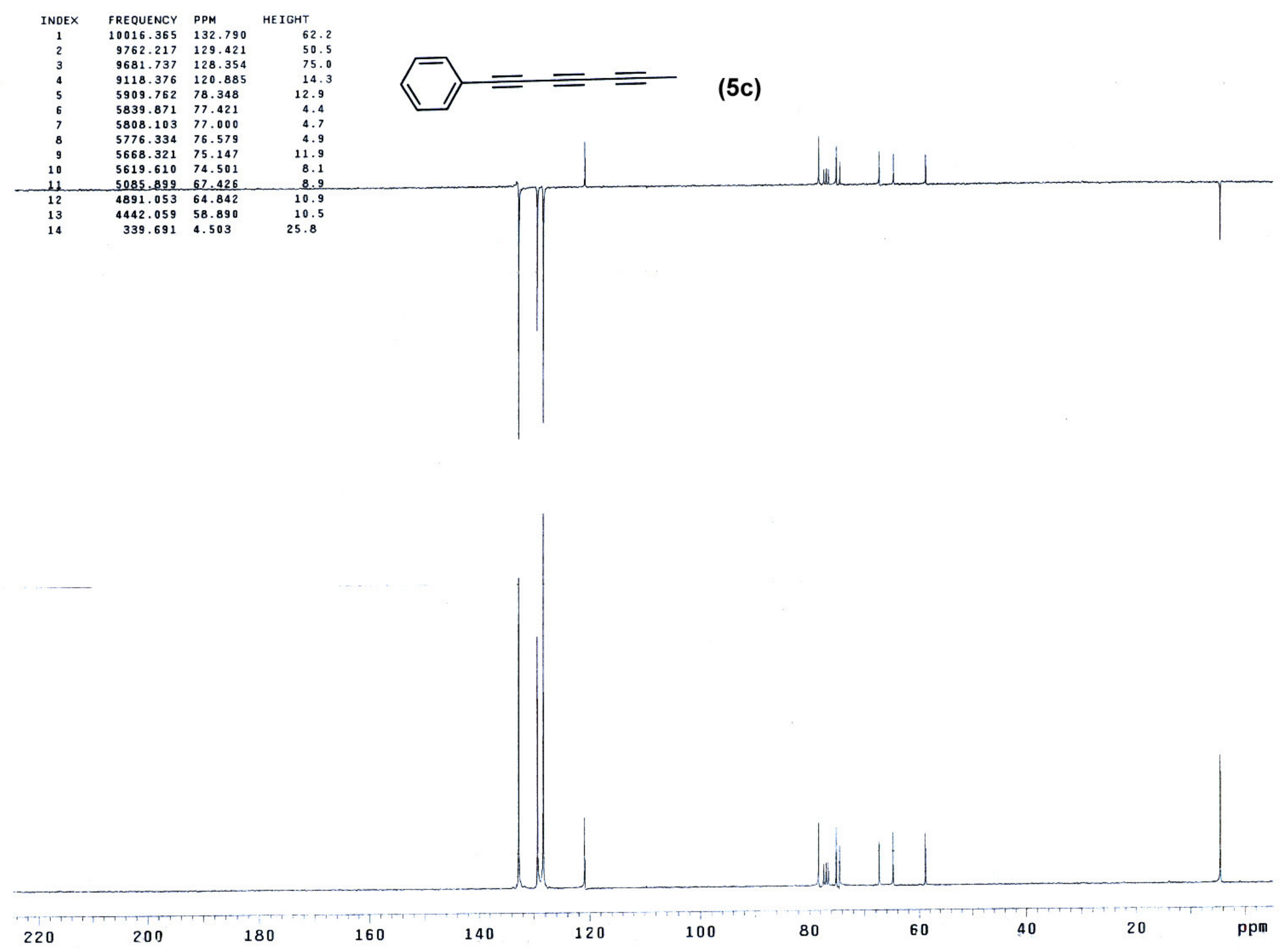
$\begin{array}{rrlr}\text { INOEX } & \text { FREQUENCY } & \text { PPM } & \text { HEIGHT } \\ 1 & 588.869 & 1.963 & 33.3\end{array}$

$\begin{array}{lrrr}1 & 588.869 & 1.963 & 33.3 \\ 2 & 56.326 & 0.188 & 75.0\end{array}$

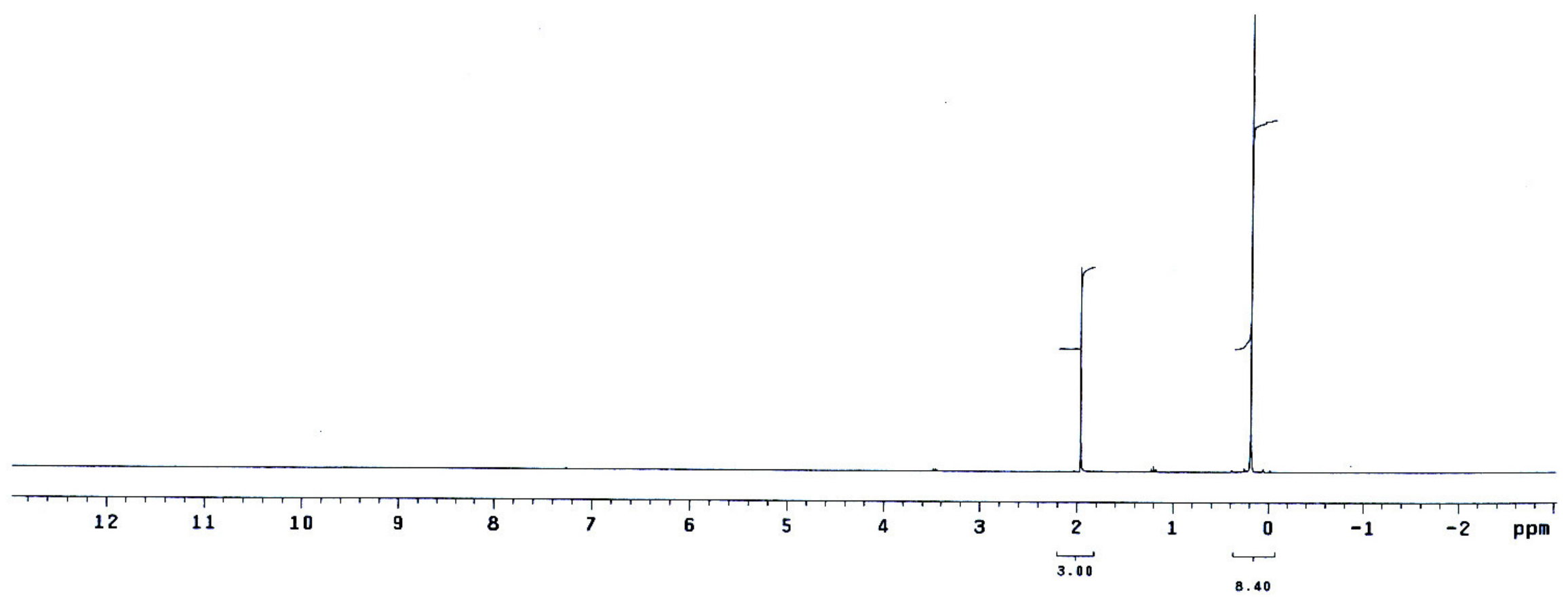



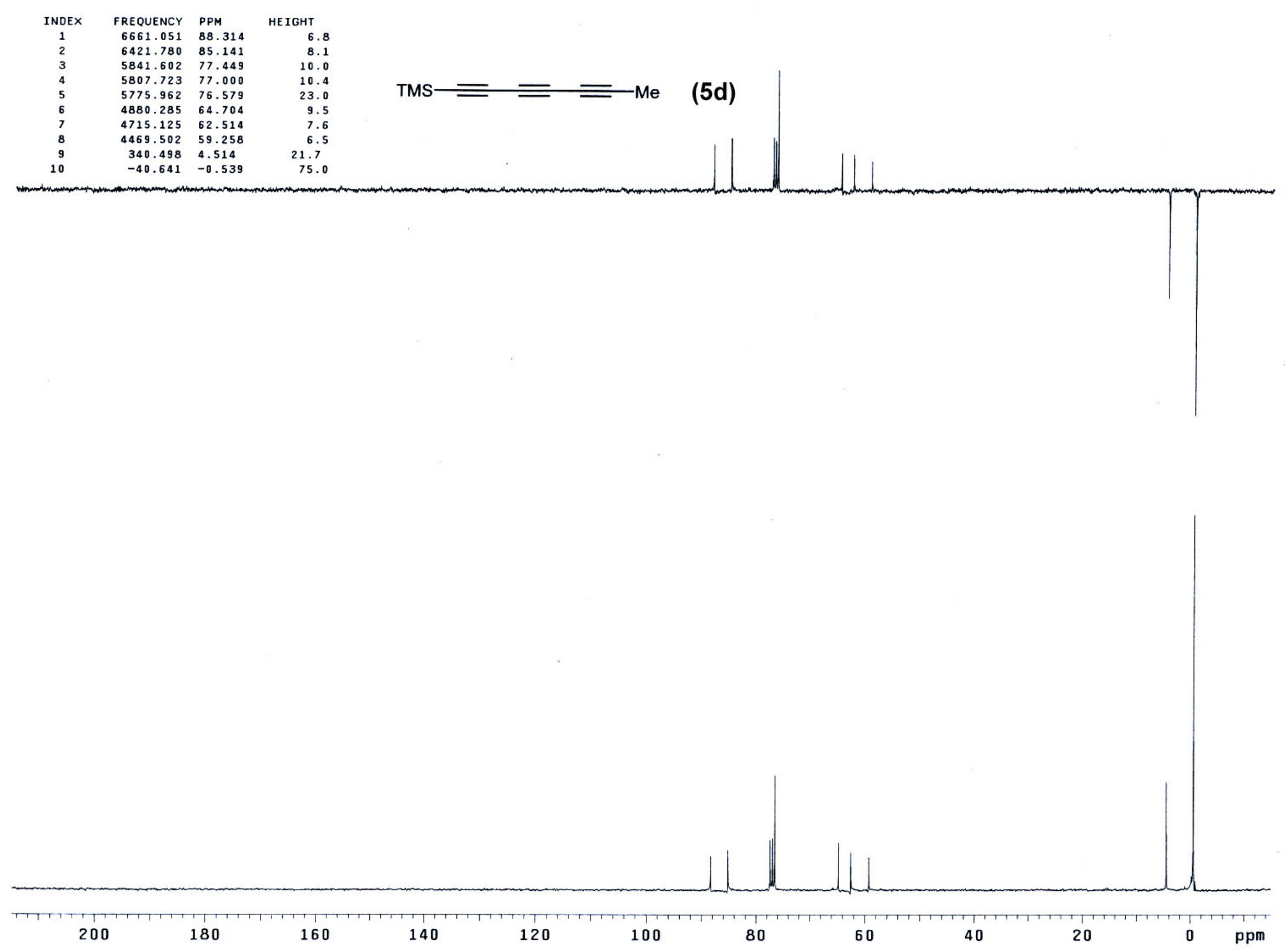


$\begin{array}{crlr}\text { INDEX } & \text { FREQUENCY } & \text { PPM } & \text { HEIGHT } \\ 1 & 2279.117 & 7.598 & 18.5 \\ 2 & 2272.086 & 7.574 & 25.3 \\ 3 & 2238.690 & 7.463 & 9.7 \\ 4 & 2236.347 & 7.455 & 3.8 \\ 5 & 2232.832 & 7.443 & 10.3 \\ 6 & 2231.660 & 7.439 & 10.5 \\ 7 & 2224.629 & 7.416 & 20.7 \\ 8 & 2218.184 & 7.394 & 18.9 \\ 9 & 2217.013 & 7.391 & 20.7 \\ 10 & 2209.982 & 7.367 & 7.5 \\ 11 & 620.461 & 2.068 & 100.0\end{array}$

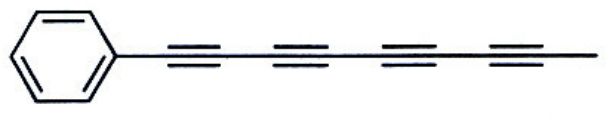

(7a)

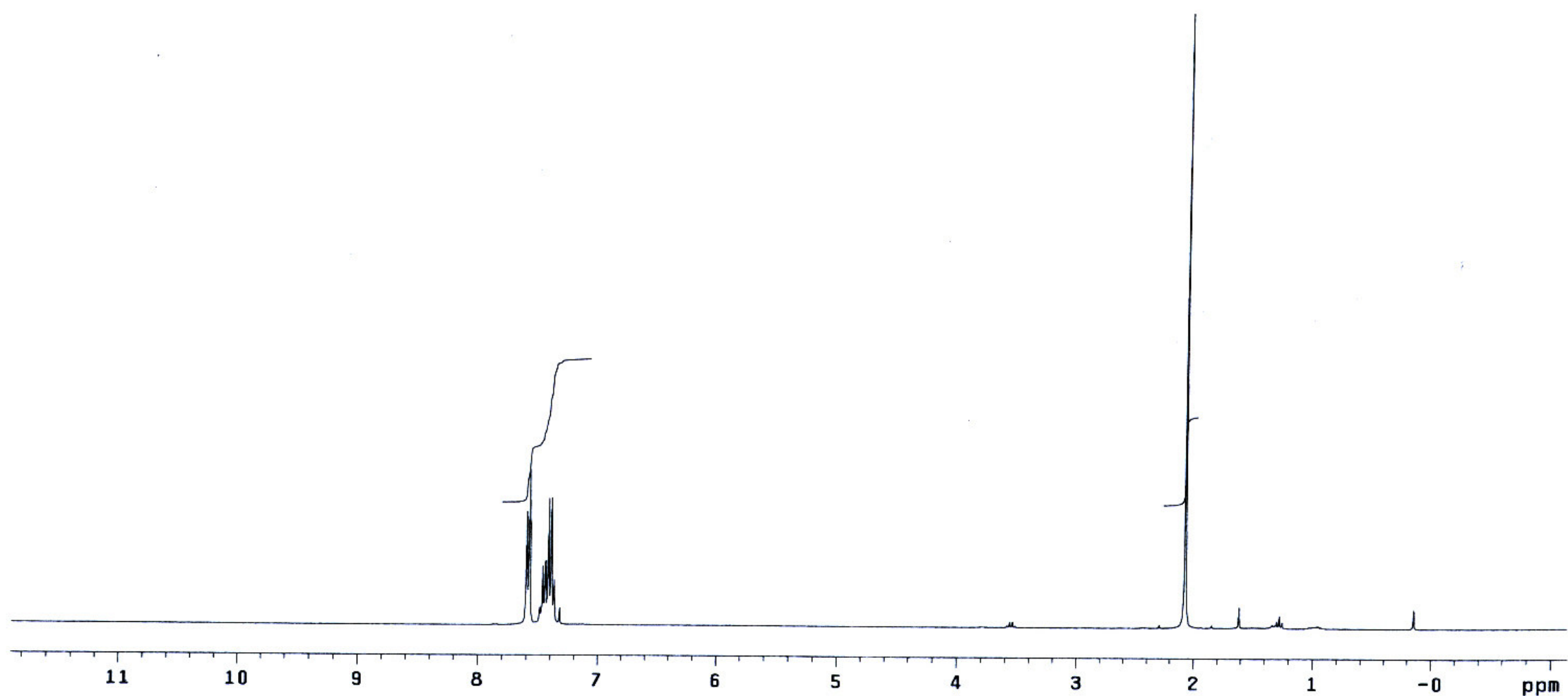




$\begin{array}{crlc}\text { INDEX } & \text { FREQUENCY } & \text { PPM } & \text { HEIGHT } \\ 1 & 10043.896 & 133.155 & 65.5 \\ 2 & 9796.102 & 129.870 & 50.2 \\ 3 & 9692.325 & 128.494 & 75.0 \\ 4 & 9092.960 & 120.549 & 12.3 \\ 5 & 5873.756 & 77.870 & 15.8 \\ 6 & 5839.870 & 77.421 & 38.0 \\ 7 & 5808.102 & 77.000 & 41.5 \\ 8 & 5776.333 & 76.579 & 43.1 \\ 9 & 5729.739 & 75.961 & 15.6 \\ 10 & 5617.491 & 74.473 & 10.7 \\ 11 & 5096.488 & 67.566 & 11.3 \\ 12 & 4899.524 & 64.955 & 12.6 \\ 13 & 4884.698 & 64.758 & 13.4 \\ 14 & 4539.481 & 60.181 & 13.0 \\ 15 & 4501.359 & 59.676 & 11.9 \\ 16 & 354.516 & 4.700 & 29.1\end{array}$

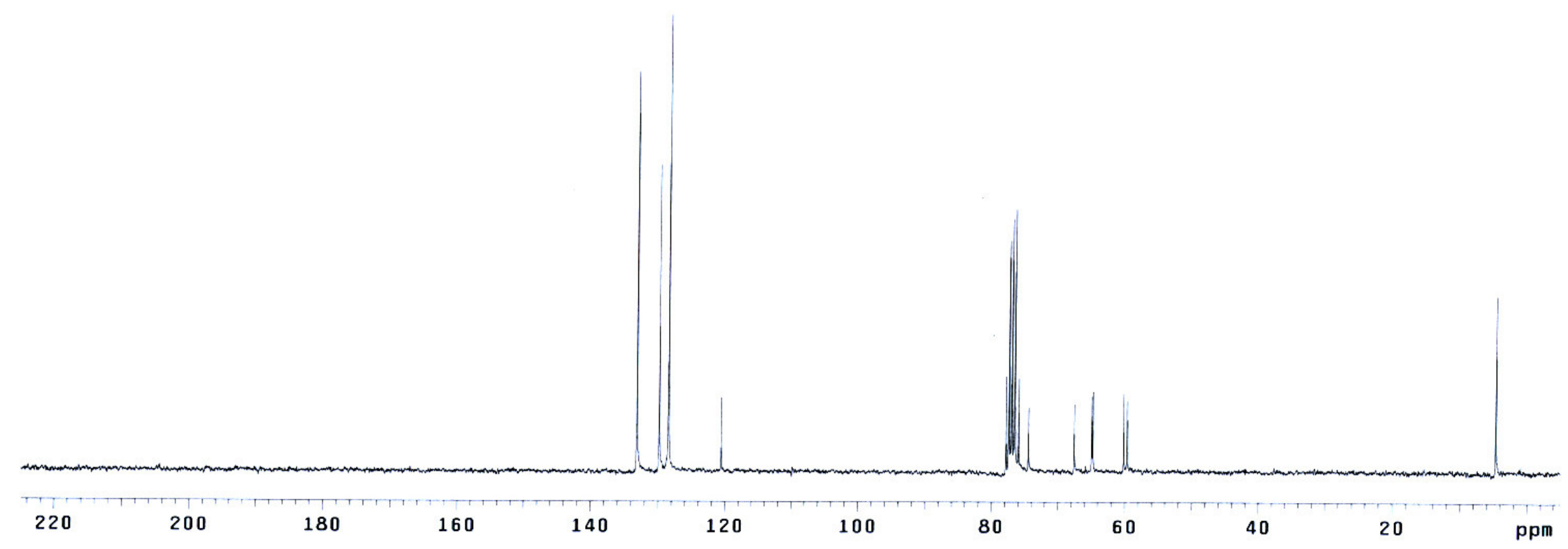



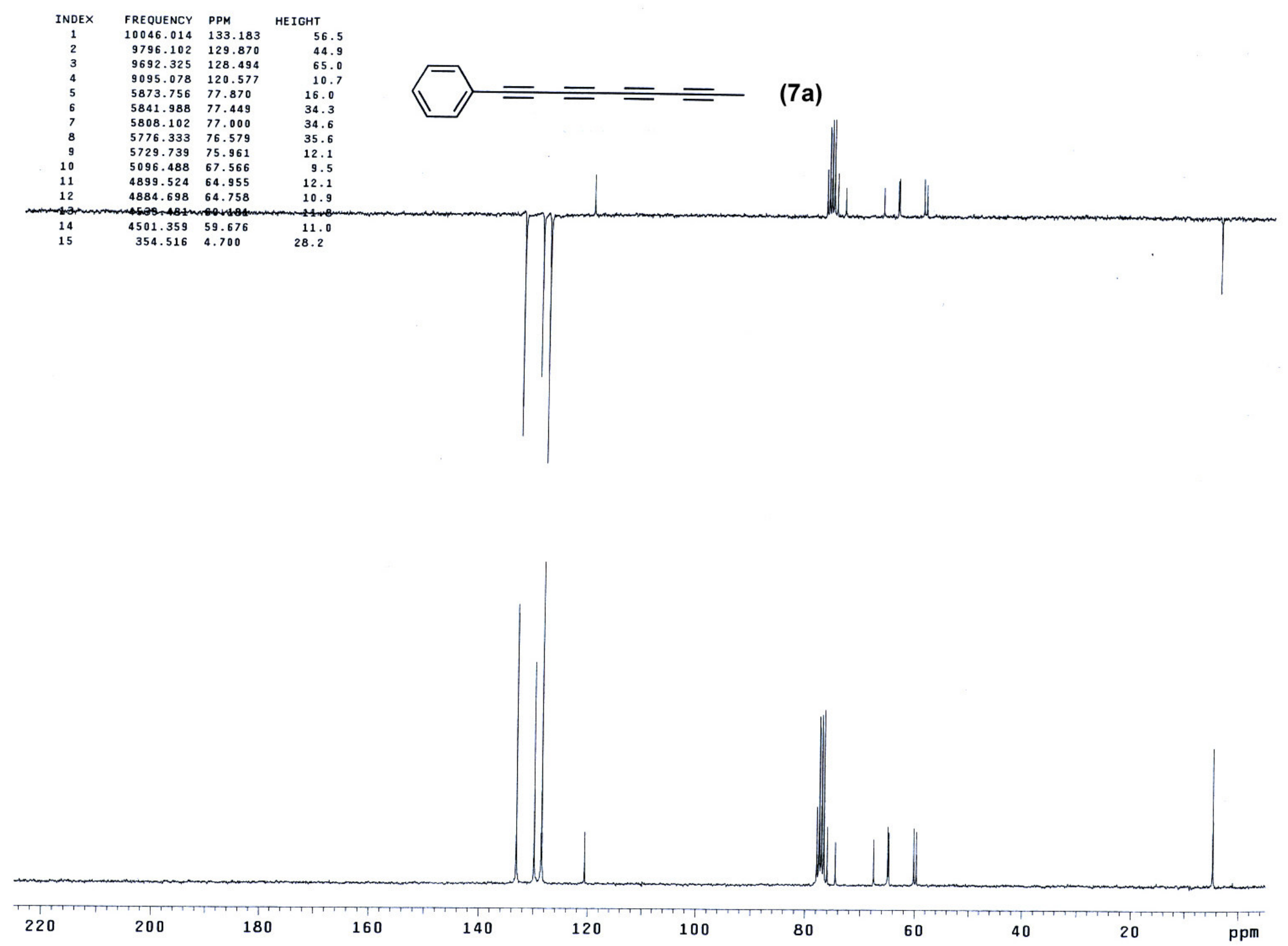


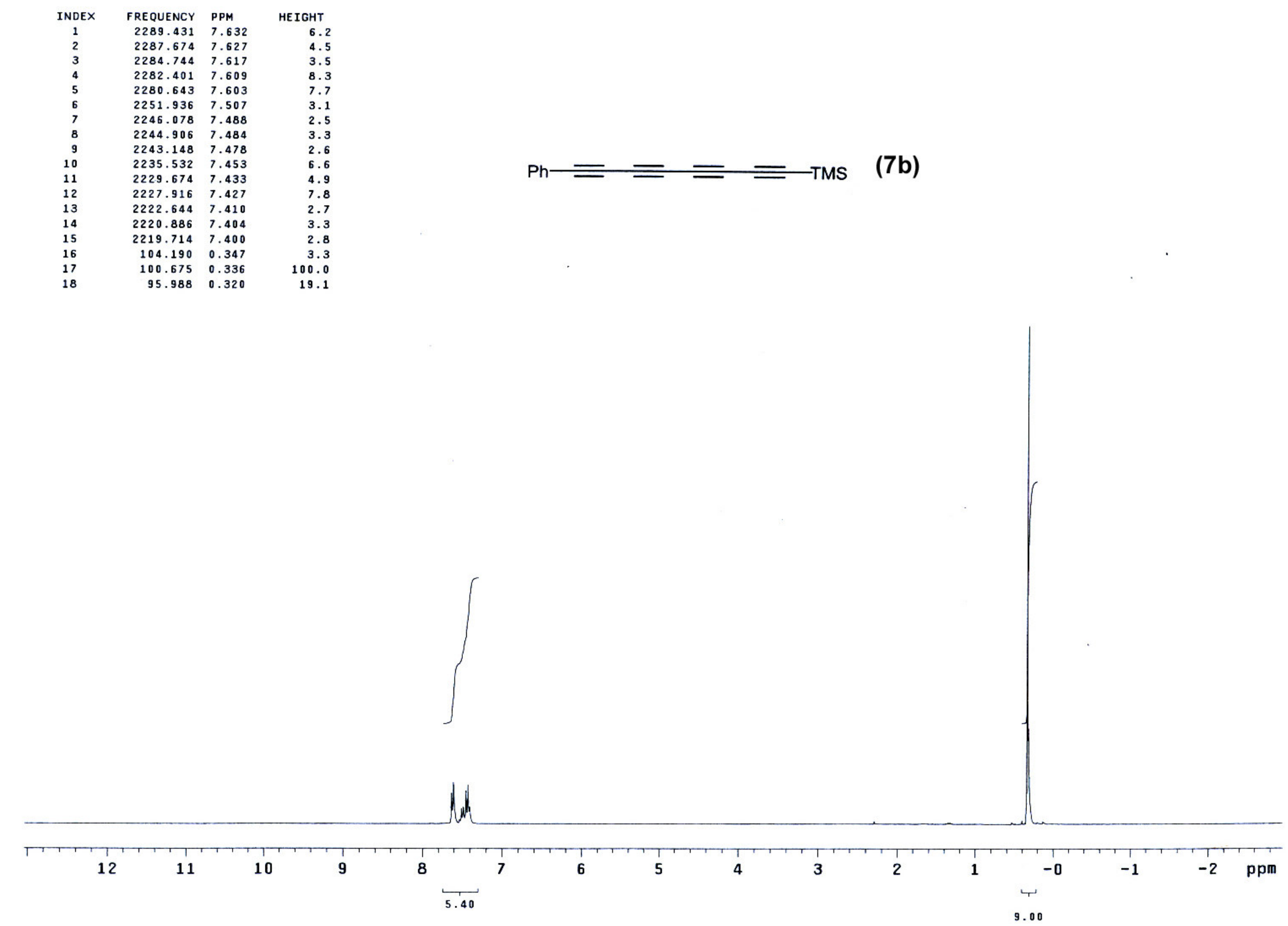




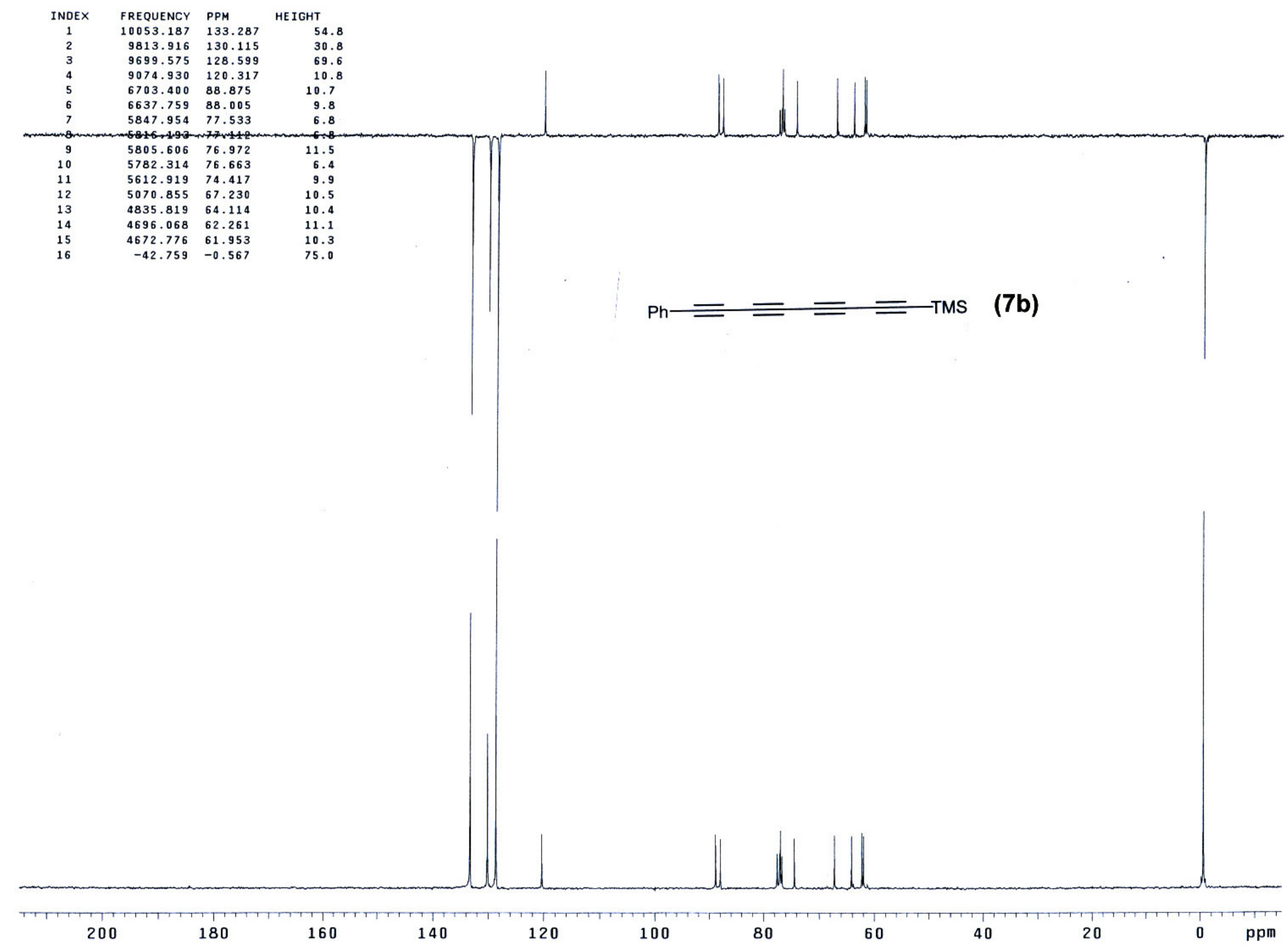




$\begin{array}{crlr}\text { INDEX } & \text { FREQUENCY } & \text { PPM } & \text { HEIGHT } \\ 1 & 2211.019 & 7.371 & 17.2 \\ 2 & 2202.817 & 7.344 & 21.5 \\ 3 & 2134.272 & 7.115 & 19.1 \\ 4 & 2126.655 & 7.090 & 16.4 \\ 5 & 2015.929 & 6.721 & 19.9 \\ 6 & 695.410 & 2.318 & 75.0 \\ 7 & 0.000 & 0.000 & 14.4 \\ 8 & -1.172 & -0.004 & 8.0\end{array}$
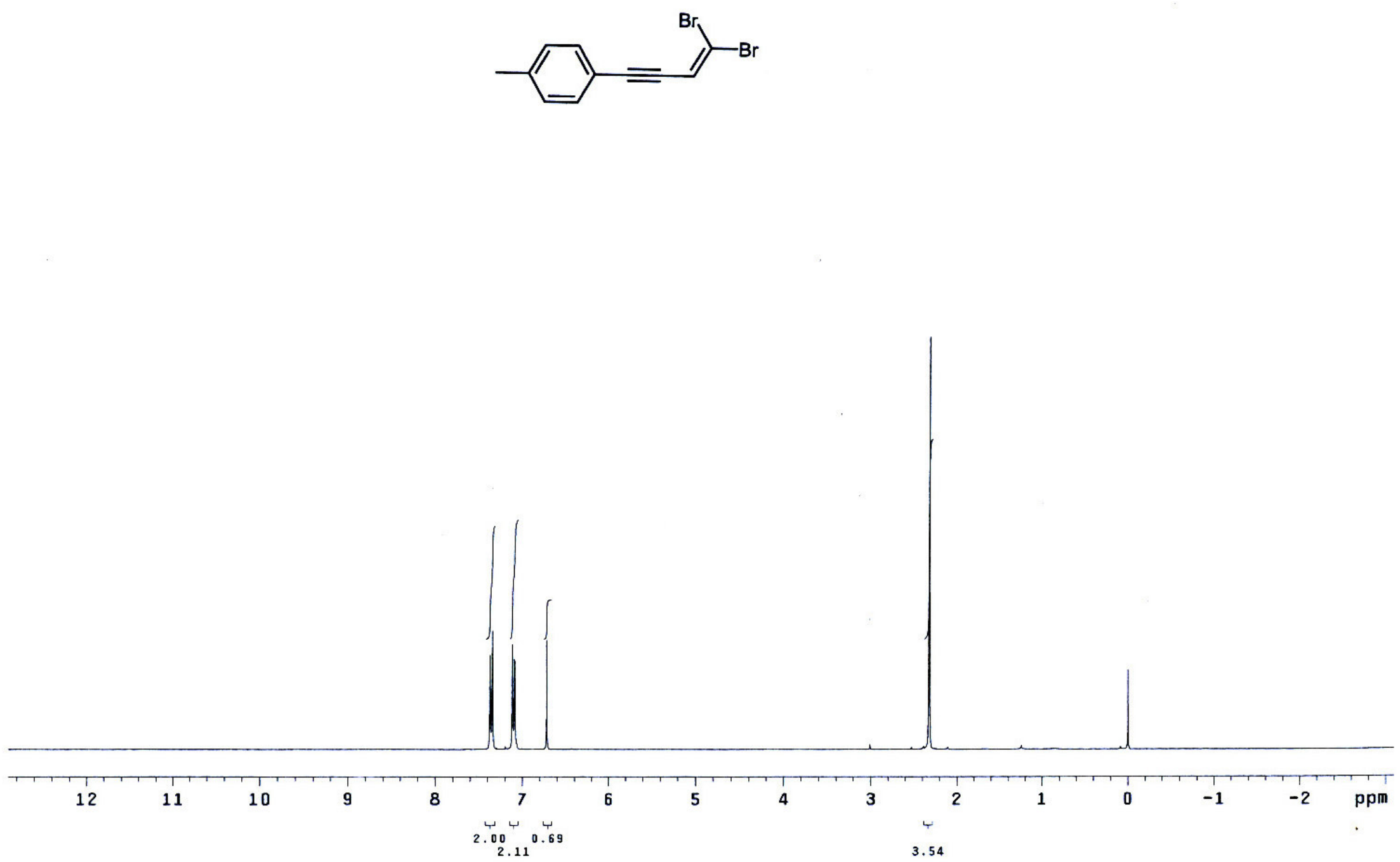

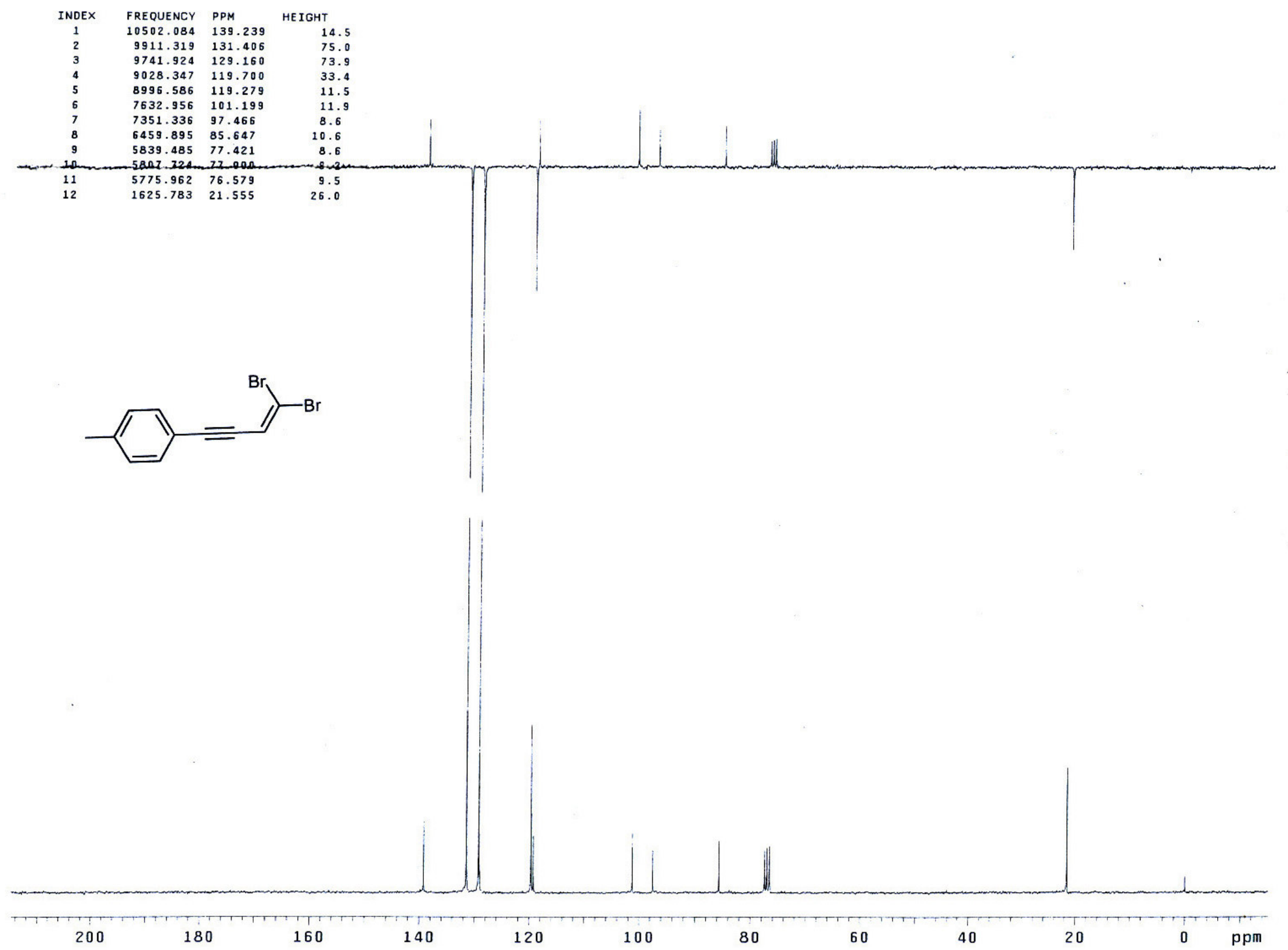


$\begin{array}{crlr}\text { INDEX } & \text { FREQUENCY } & \text { PPM } & \text { HEIGHT } \\ 1 & 2255.633 & 7.519 & 16 \\ 2 & 2253.876 & 7.513 & 20 . \\ 3 & 2250.360 & 7.502 & 18 . \\ 4 & 2249.189 & 7.498 & 18 . \\ 5 & 224.259 & 7.488 & 20 \\ 6 & 2202.903 & 7.344 & 40 \\ 7 & 2201.146 & 7.338 & 39 . \\ 8 & 2197.630 & 7.326 & 22 . \\ 9 & 2191.771 & 7.306 & 5 \\ 10 & 195.618 & 6.553 & 30 . \\ 11 & 1358.635 & 4.529 & 75 . \\ 12 & 282.354 & 0.941 & 322 . \\ 13 & 47.998 & 0.160 & 221 .\end{array}$

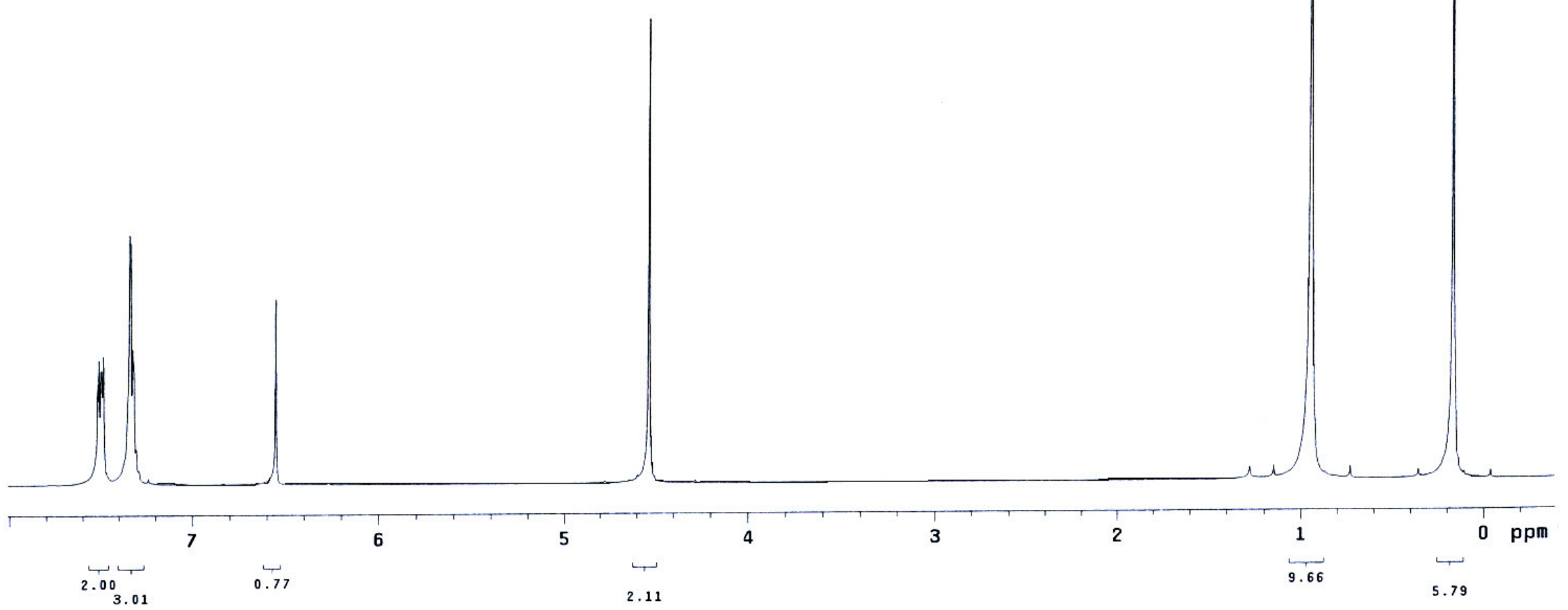



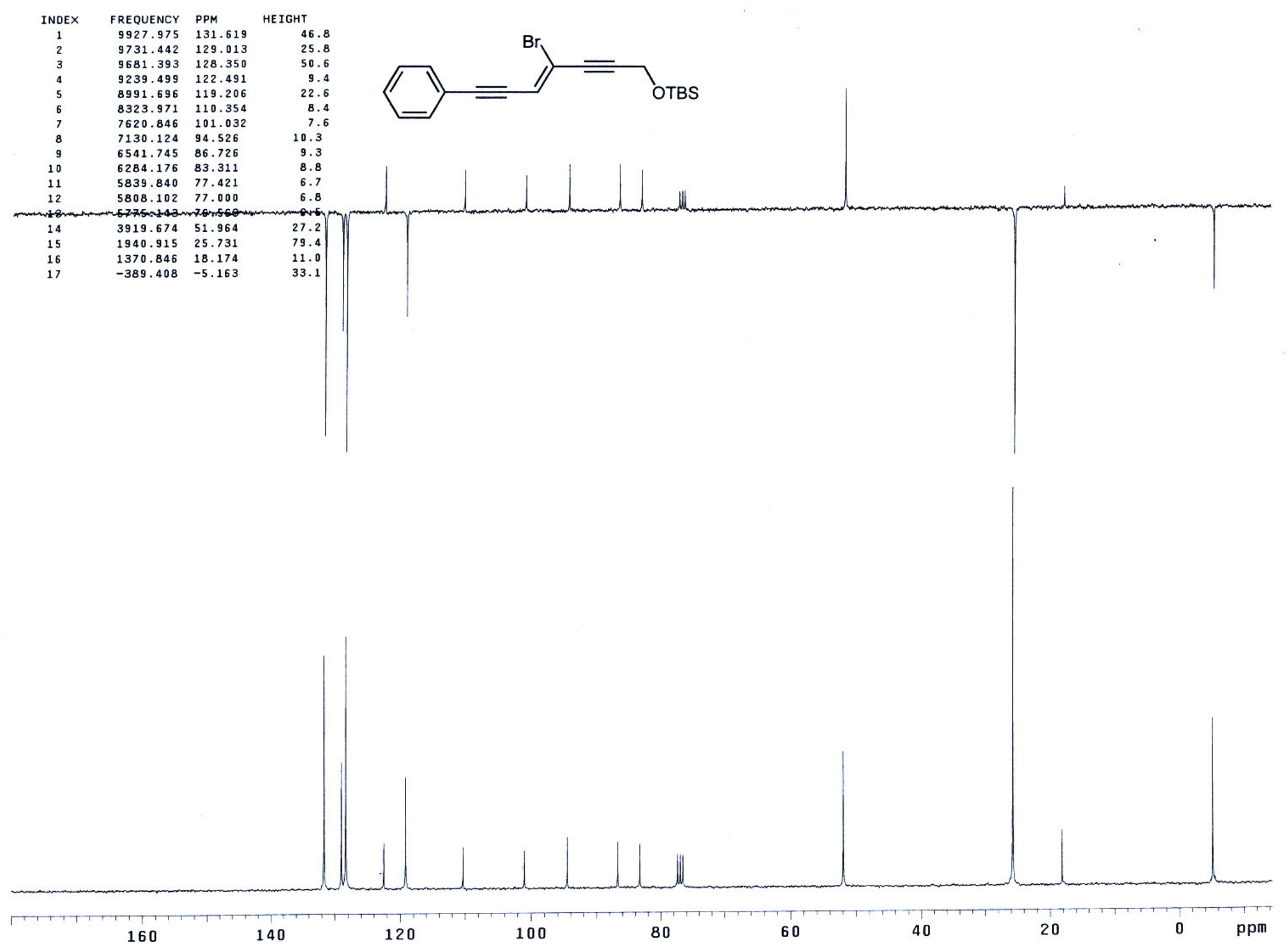

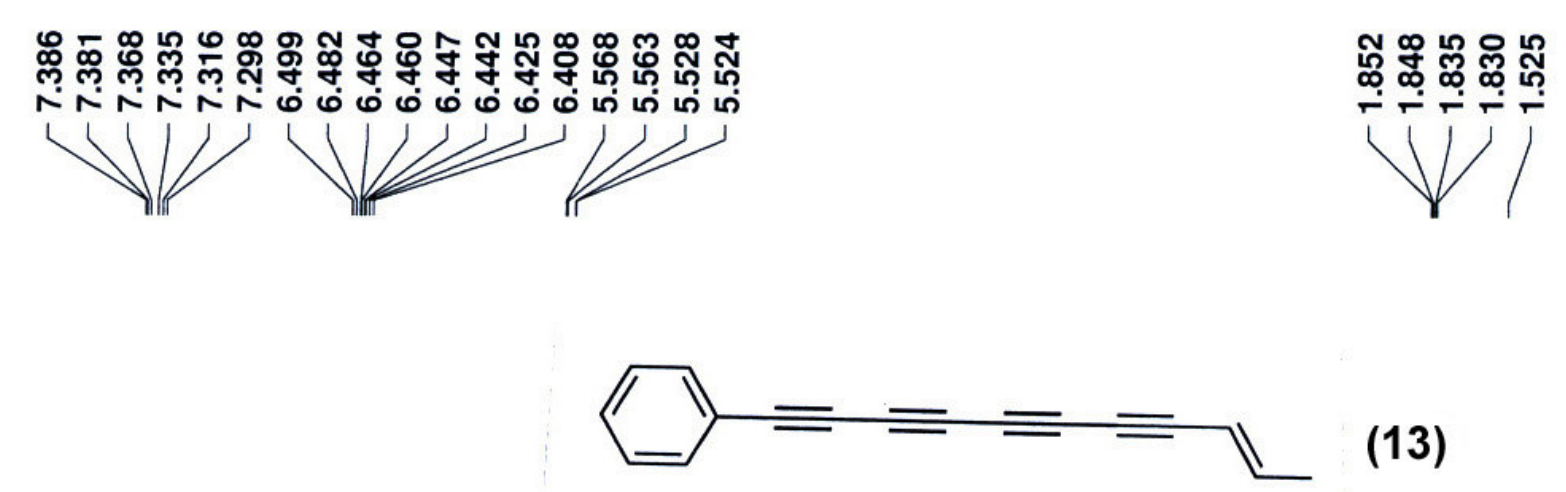

(13)

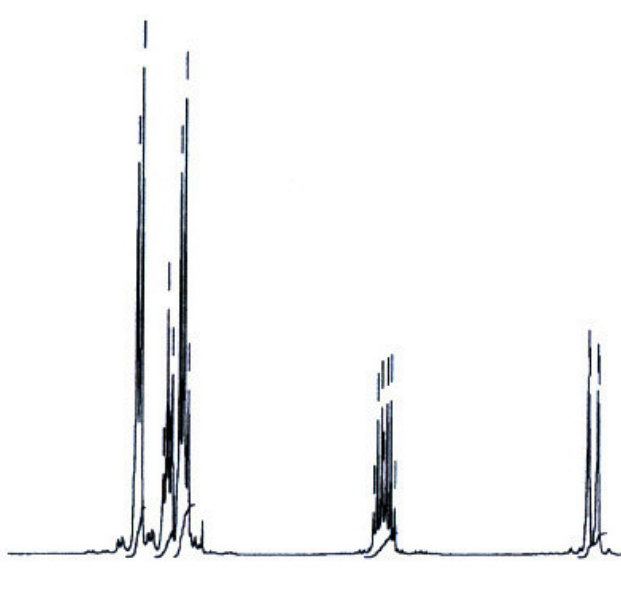

8

7

6

5

4

$\mid$

$\left|\begin{array}{l}n \\ \hat{\sigma} \\ 0\end{array}\right|$

2

| 

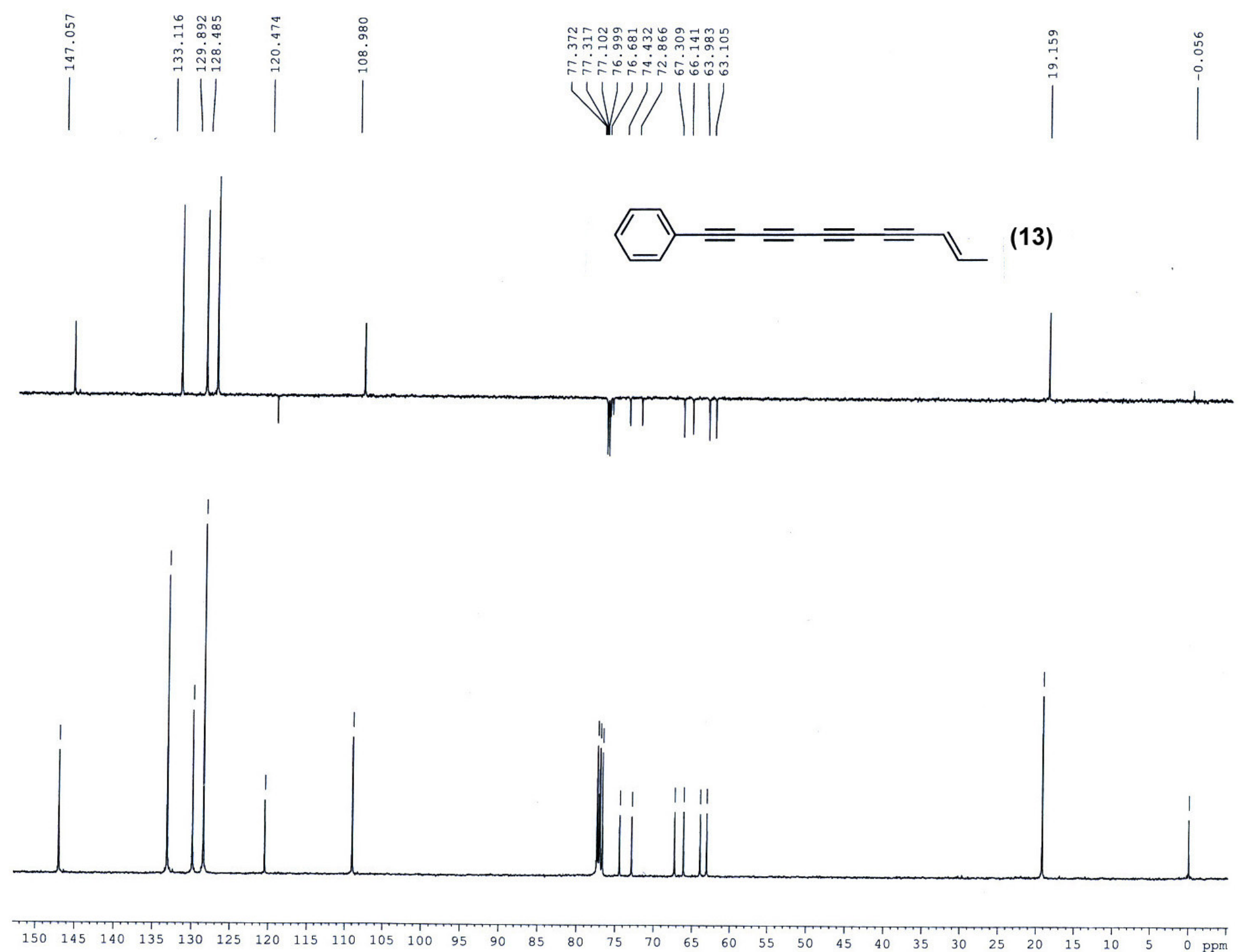


$\begin{array}{crlr}\text { INDEX } & \text { FREQUENCY } & \text { PPM } & \text { HEIGHT } \\ 1 & 2249.685 & 7.500 & 25.3 \\ 2 & 2242.655 & 7.477 & 32.0 \\ 3 & 2241.483 & 7.473 & 27.6 \\ 4 & 2219.221 & 7.398 & 29.7 \\ 5 & 2211.604 & 7.373 & 41.1 \\ 6 & 2194.615 & 7.316 & 28.2 \\ 7 & 2186.999 & 7.291 & 30.7 \\ 8 & 2133.686 & 7.113 & 33.5 \\ 9 & 2125.484 & 7.086 & 28.3 \\ 10 & 699.511 & 2.332 & 100.0 \\ 11 & 0.000 & 0.000 & 20.5\end{array}$
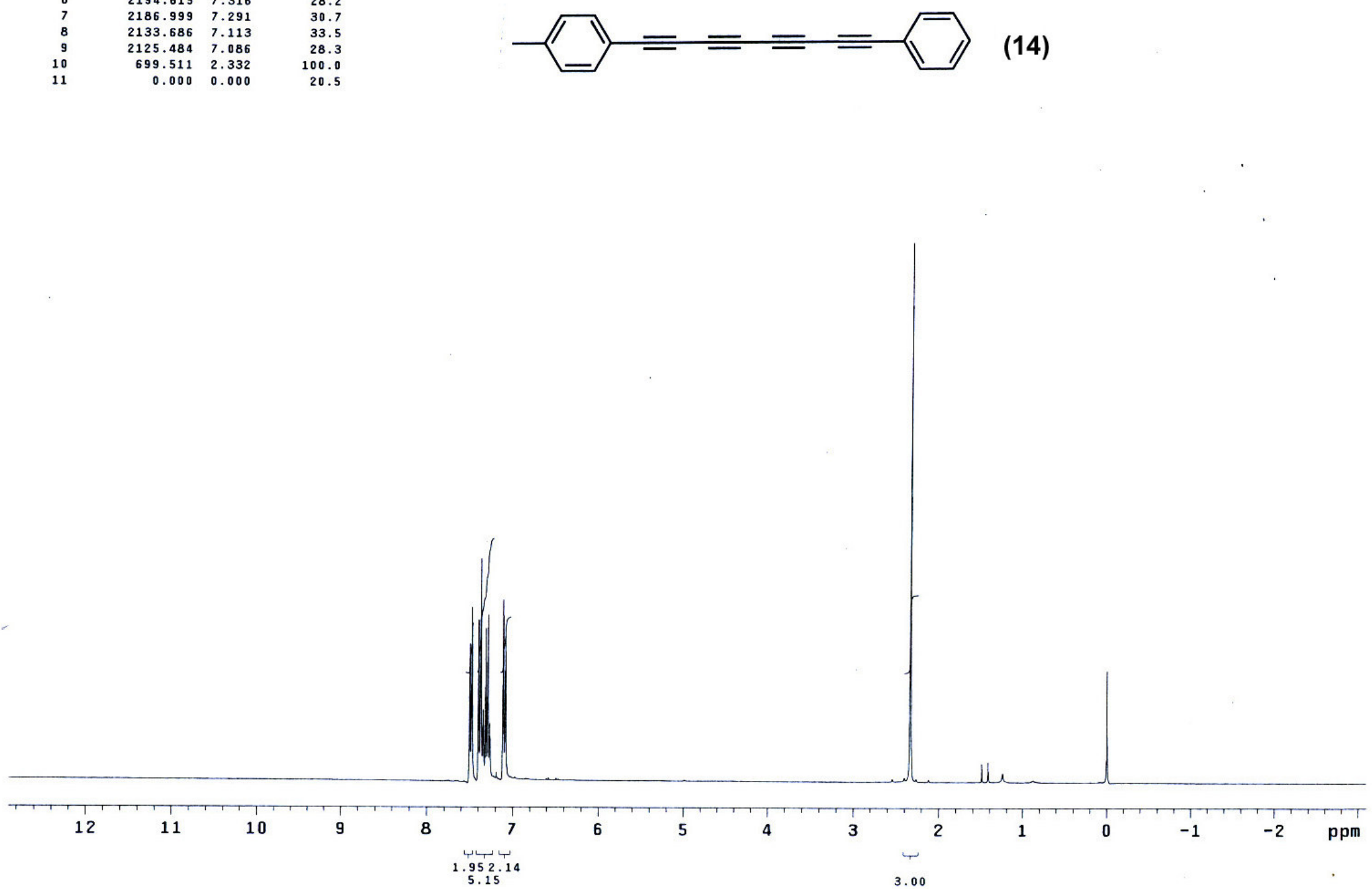


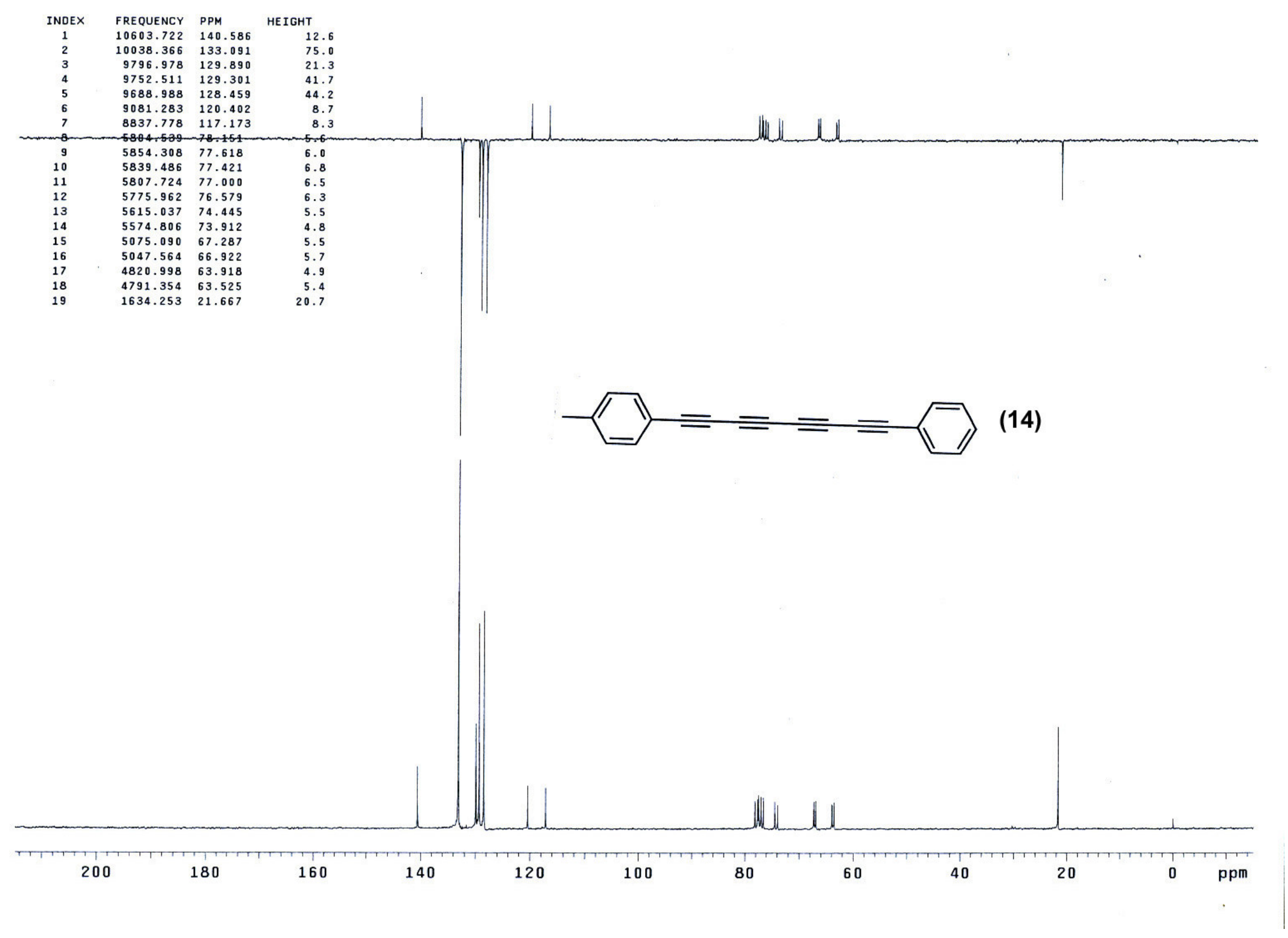



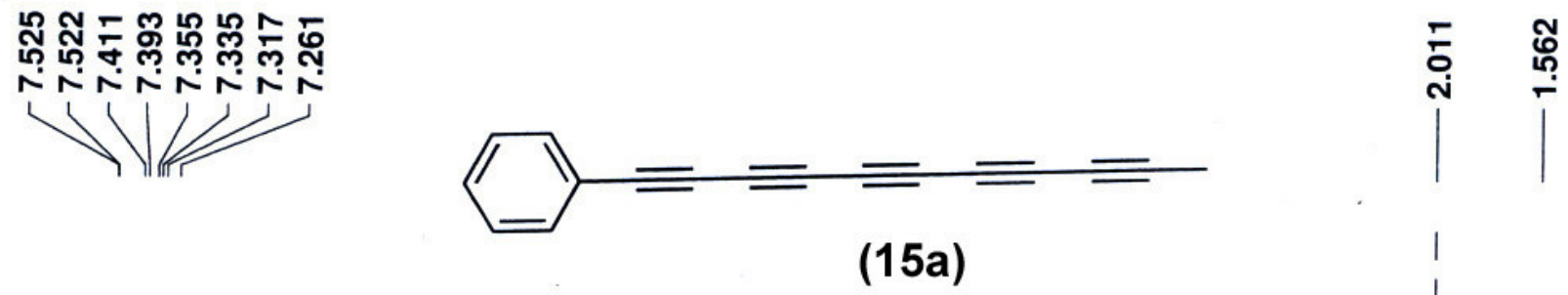

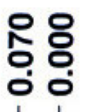

(15a)
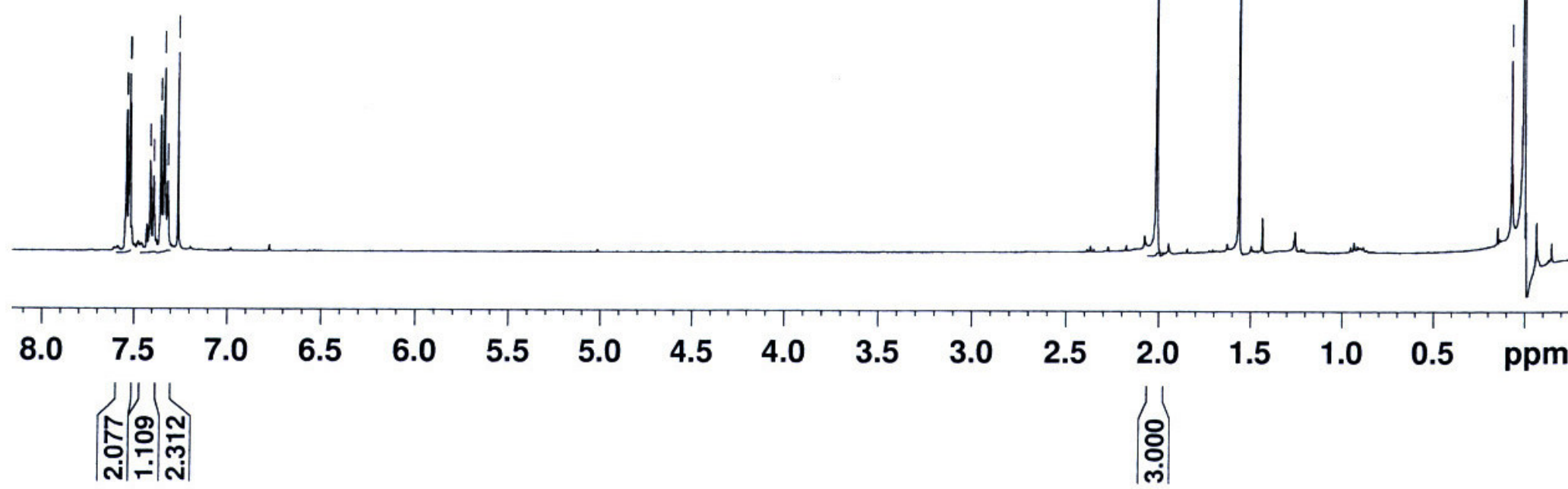


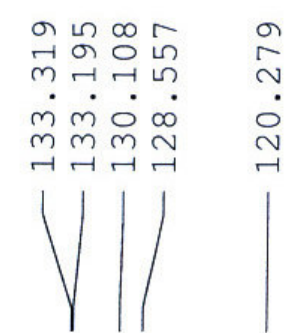

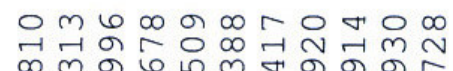

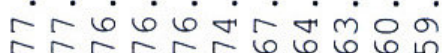

$1|1| 1 \mid$

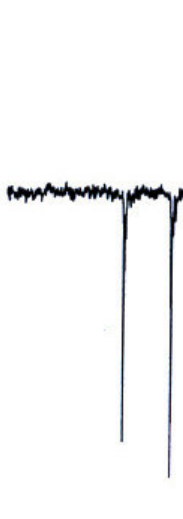

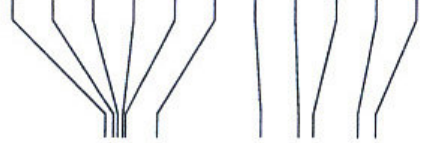

\section{II}
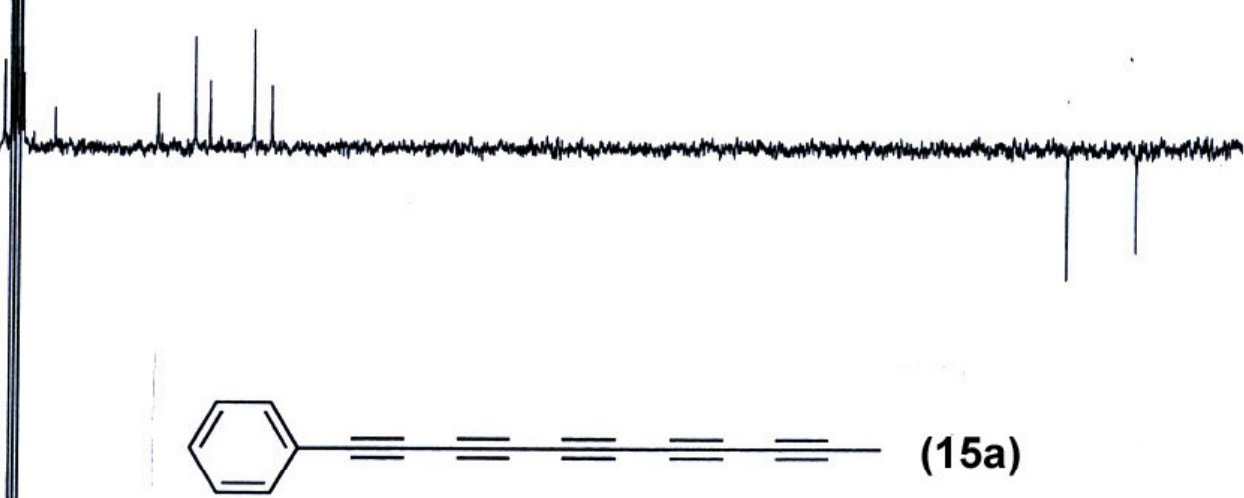

(15a)

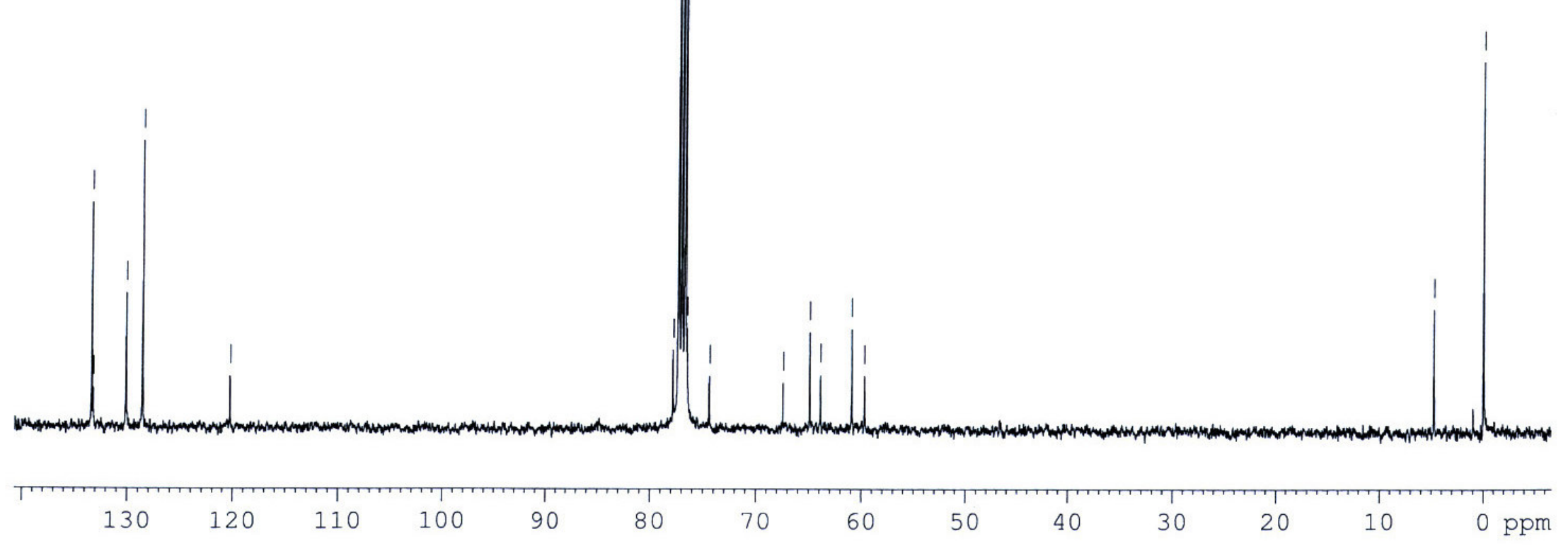




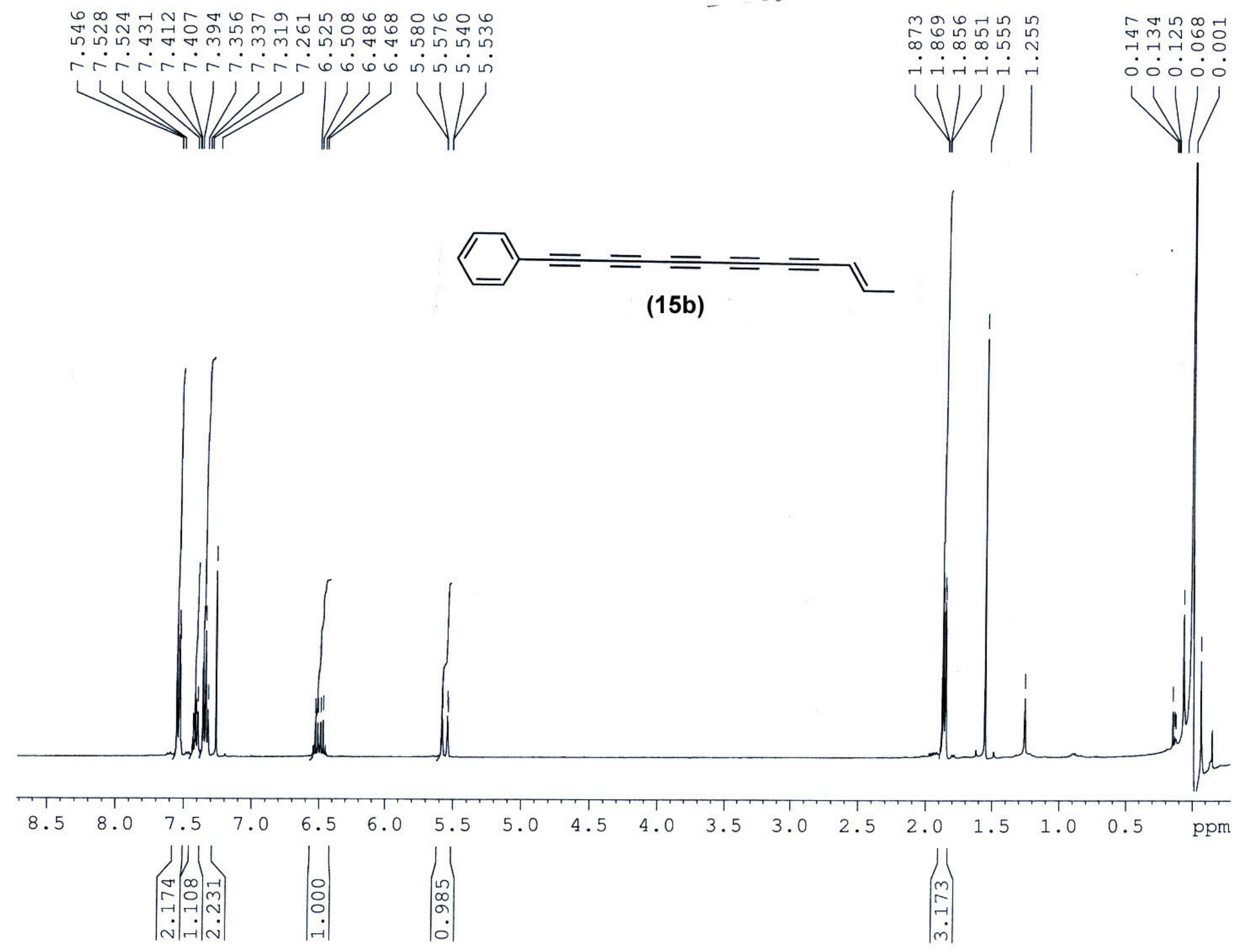




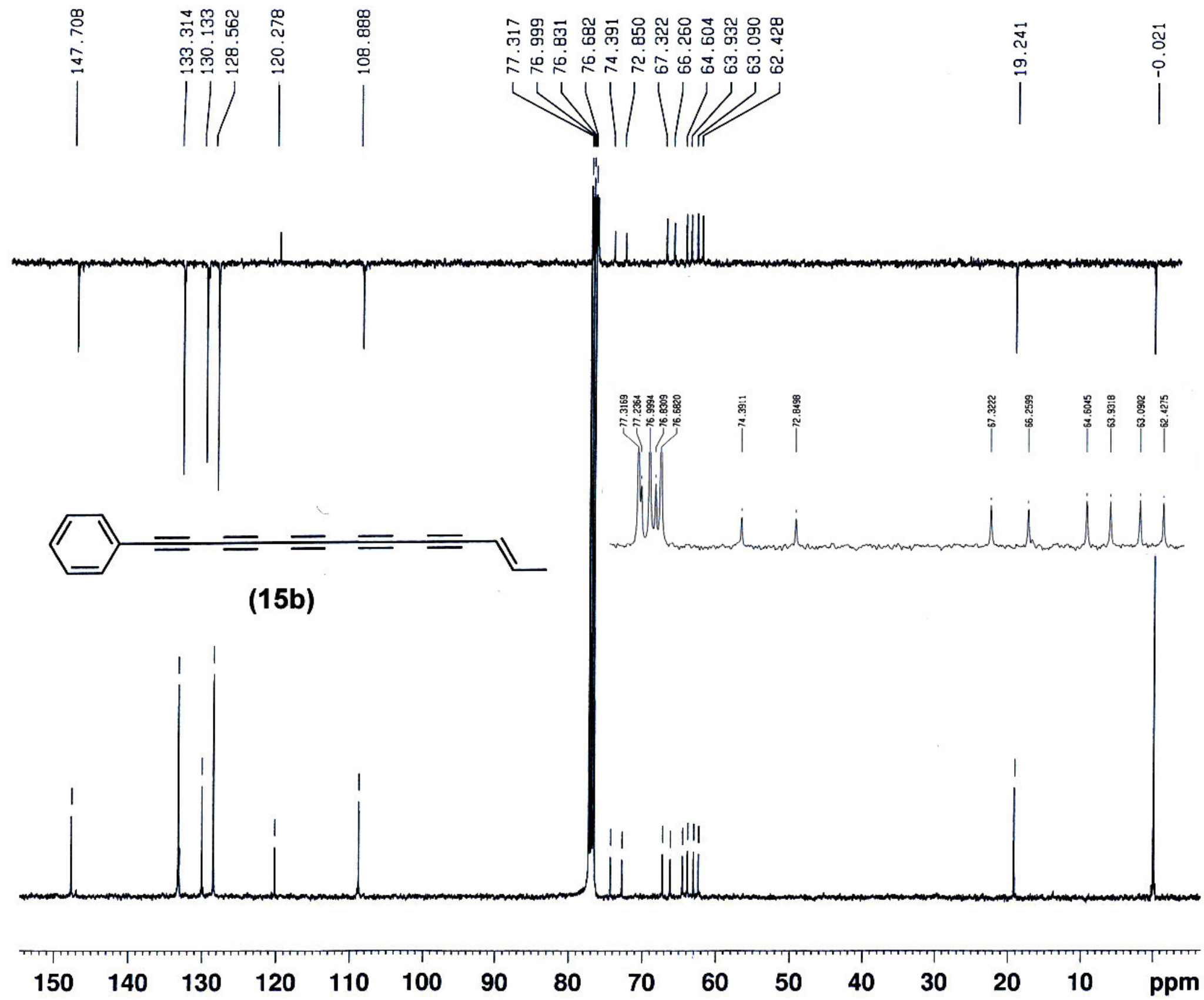



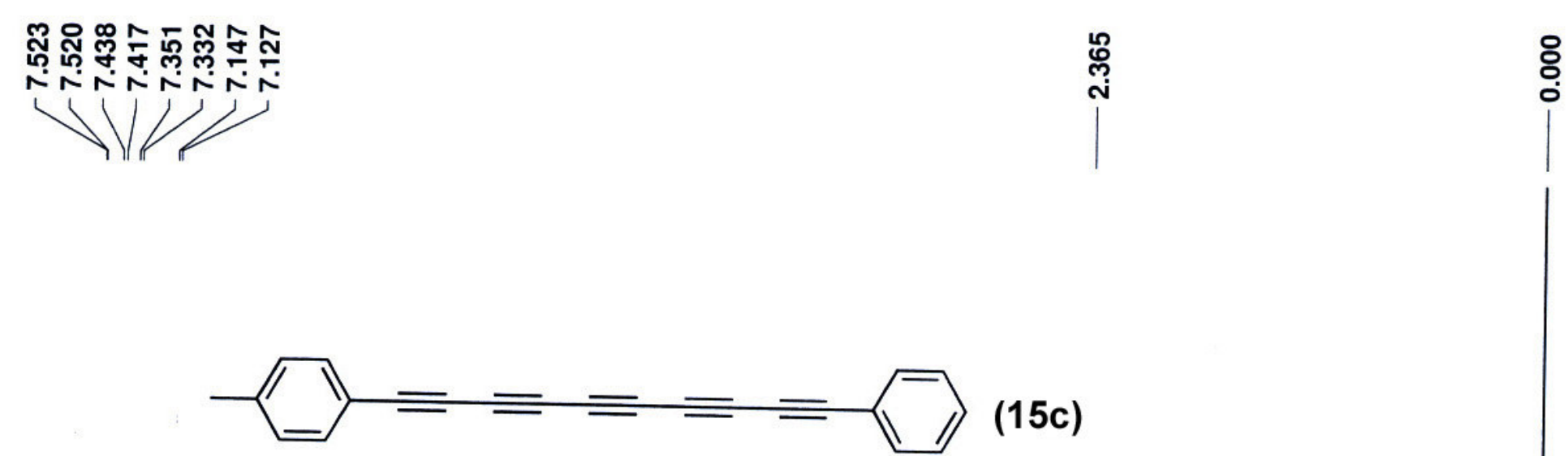

(15c)

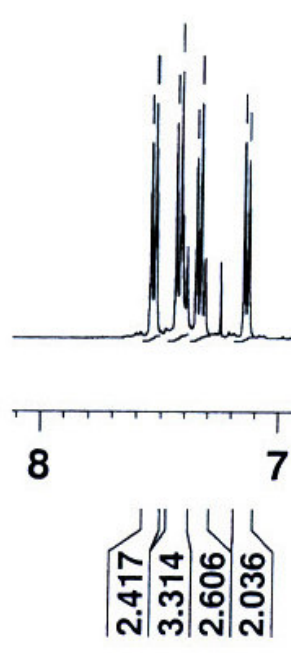

6

5
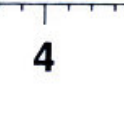

ర్లి 


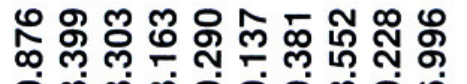

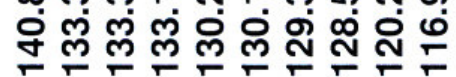
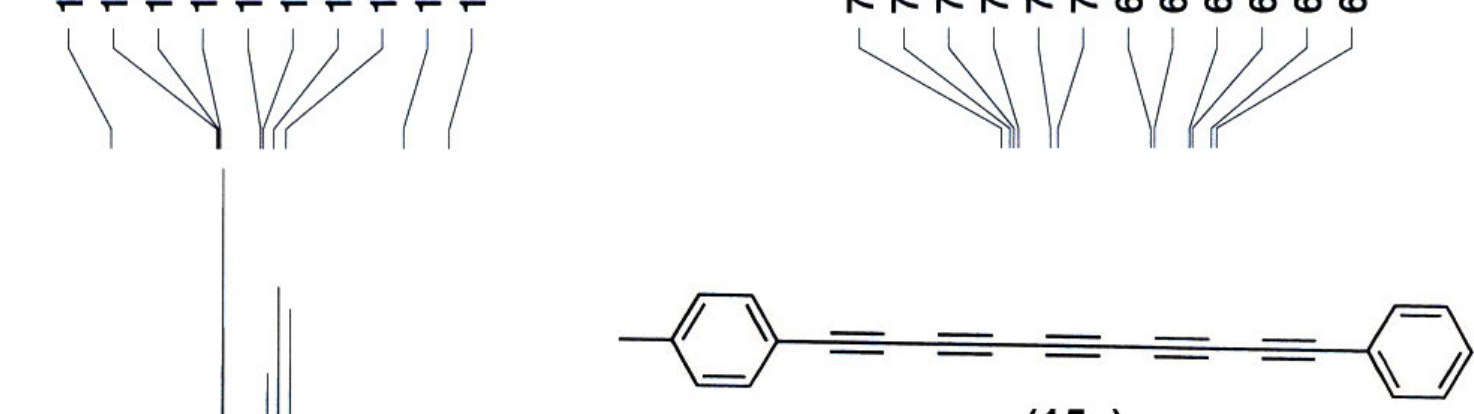

(15c)

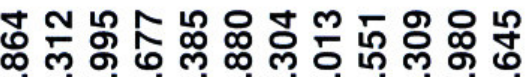

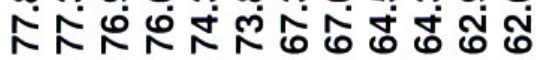
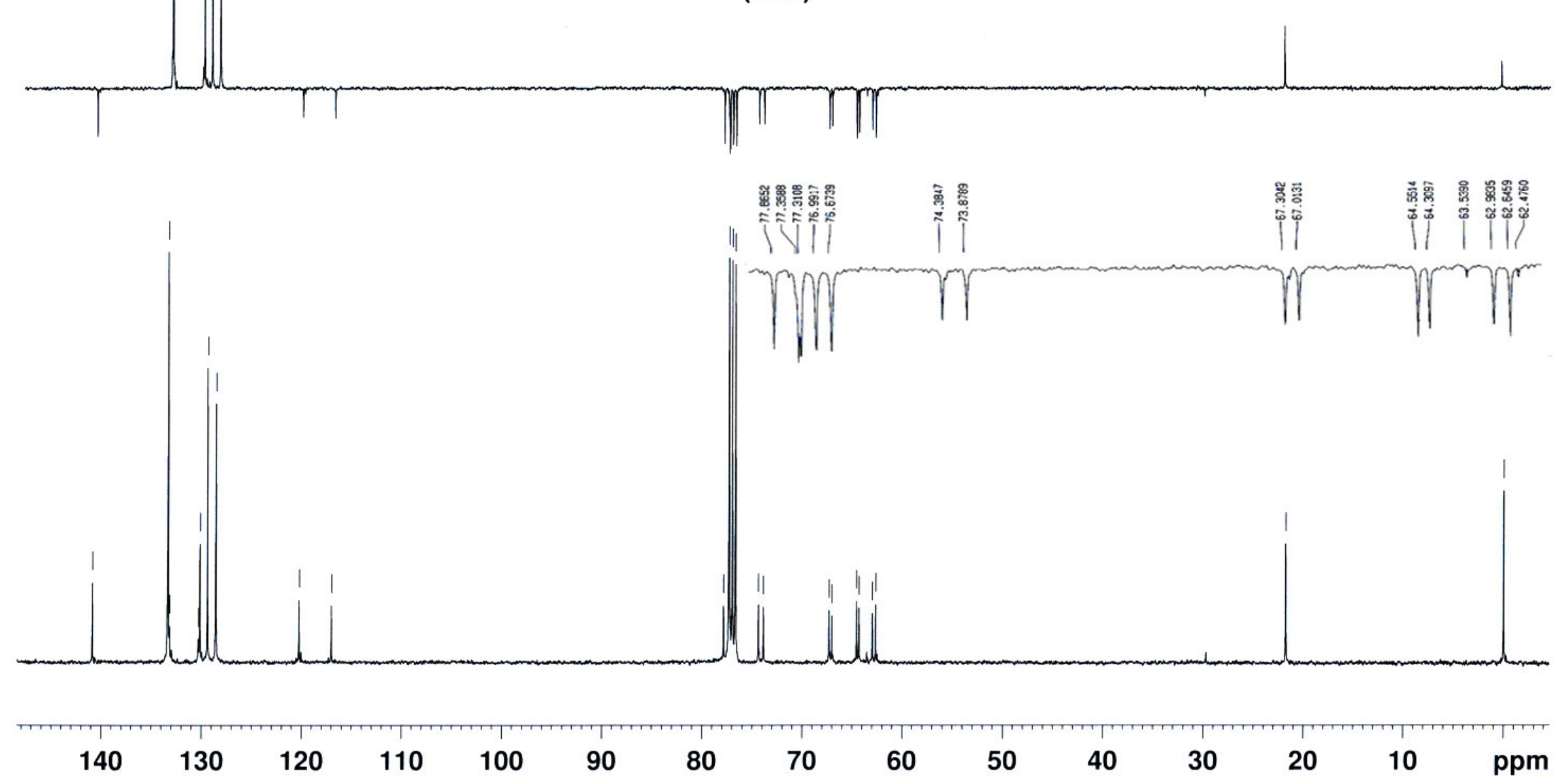